\title{
FORMULA FOR THE INDUCTANCE OF A HELIX MADE WITH WIRE OF ANY SECTION
}

\author{
By Chester Snow
}

ABSTRACT

The formula for the direct-current inductance of a single-layer helix which is here obtained takes account of the helical shape of the wire and hence of the axial component of current. It does not assume uniform current density over the section of the wire, but is correct to the second order for any current distribution which may be expanded by Maclaurin's theorem over the section of the wire. It is especially simple if this section is symmetrical about two axes through its center of gravity. It is correct to the second order inclusive in $\frac{p}{a}$ where $2 \pi p$ is the pitch of the helix and $a$ is the radius of the cylindrical form. The terms neglected are of the order of $\left(\frac{p}{a}\right)^{8} \log \frac{p}{a}$. The formula is derived from the fundamental equations of the magnetic field and naturally takes account of the periodic structure of the field, which is important in the vicinity of the wires. This periodic part of the field, which is due to the discrete nature of the windings and to their regular spacing, falls off rapidly (exponentially) as the distance from the wire increases, so that, at appreciable distances, the field assumes its regular value due to a current sheet. This current sheet differs from Lorentz's in that it has an axial as well as a circular component of current. Attention is also given to the mutual inductance of the helix and the return leads.

The inductance of a helix (aside from the leads or their mutual inductance) is given in the form

$$
L_{\mathbf{h}}=\int \mathcal{S} u\left(x_{1} y_{1}\right) d S_{1} \mathcal{S} \mathcal{S} u\left(x_{2} y_{2}\right) d S_{2} M\left(x_{1} y_{1} x_{2} y_{2}\right)
$$

where the integration is performed twice over any section of the wire (say, its initial section) where $u(x y)$ is the magnitude of the current density at a point $x y$ of this section, and where $M\left(x_{1} y_{1} x_{2} y_{2}\right)$ is a symmetrical function of the two points $P\left(x_{1} y_{1}\right)$ and $P_{2}\left(x_{2} y_{2}\right)$ in this section. It represents the mutual inductance between two unit current helical filaments of the same pitch $2 \pi p$ and axial length $l=2 \pi p N$ which pass through these two points. By studying this function and making a number of reductions, an approximation to $M$ is obtained, correct to the second order in $\frac{p}{a}, \frac{x_{2}-x_{1}}{a}$ and $\frac{y_{2}-y_{1}}{a}$. It is then an easy matter to obtain the inductance by surface integration over the section of the wire for ordinary kinds of sections. Formulas for circular and rectangular wires are obtained and simplified, equations (114), (120), (131).

The paper also contains (in Appendix 10) the exact solution for the field of a helical current filament, infinite in length. 
I. Introduction.

II. Geometrical considerations

III. Distribution of steady current

IV. Fundamental electrical equations

V. Definition of the inductance of the helix.-

VII. Evaluation of $M\left(r_{1} y_{1} r_{2} y_{2}\right)$

1. Principal terms arising from $G_{0}$

2. Principal terms arising from $G_{1} \ldots 43$

3. Principal terms of $G_{2}$ and $G_{3}$

4. Collection of all terms in $M\left(x_{1} y_{1} x_{2} y_{2}\right)$

VIII. General formula for the inductance of a helical distribution of current for any shape of section of the wire

1. Wire of section $S$ symmetrical with respect to the $x$ and $y$ axes through its center of gravity

(a) Wire of circular section

(b) Wire of rectangular section.

2. Numerical examples

(a) Circular wire

(b) Square wire

3. Simplified formula for inductance of a helix with rectangular wire

1. Expansions of certain hypergeometric functions........ 479

2. Graphical method of computing $B_{0}$

3. Evaluation of $\sqrt{r_{1} r_{2}} \int_{0}^{l} d \lambda_{1} \int_{0}^{\lambda_{1}} \phi_{1}(\mu) d \lambda$ to second order...... 485

4. Second order approximation for $G_{0}(y) \ldots 492$

5. Evaluation of $G_{1}(y)$ to the second order. 493

6. The integral $\int_{0}^{\infty} f(x) J_{\mathrm{k}}\left(r_{1} x\right) J_{\mathbf{k}}\left(r_{2} x\right) d x \ldots \ldots$

7. Transformations of the function $F_{2}(z)$

8. Transformations of $F_{1}(z)$

9. Analytical method of evaluating the integral $B_{0} \ldots \ldots \ldots$

10. The magnetic field of an infinitely long helical current filament

11. Corrections due to lead-in wires.

\section{INTRODUCTION}

To compute the inductance of a coil, it is sufficient in most practical cases to proceed as if the wire completely filled all the winding space. A single-layer solenoid may be replaced by an ideal current sheet in which the windings consist of infinitely thin strips of tape without any insulating space between them. Such a process will undoubtedly give a first approximation to the inductance, but it is evident that where precision is desired a formula for the inductance should be based upon expressions which represent the magnetic field in the immediate neighborhood of the windings or (as in the present case) the vector potential within the wires themselves. 
The results here presented have been derived directly from the equations of the electromagnetic field and simplified by approximations to a preassigned order of small quantities (the second). The coil has not been idealized as a set of circles, nor as a current sheet, but cognizance has been taken of the discrete nature of the windings and the helical nature of the wire. The results are formulated for any shape of cross section of the wire and are not limited to the case of uniform-current distribution over the section.

In 1906 Rosa obtained ${ }^{1}$ a formula for the case of circular wire which has been considered sufficiently correct for the most precise of modern absolute measurements. He considered the solenoid as made up of a series of parallel circles, so that he neglected the helical shape of the wires and the axial component of current. His argument is based upon Maxwell's theorem of geometrical mean distance, which is derived from the logarithmic formula, and is strictly applicable only to infinitely long cylindrical conductors, and hence approximately correct for coils whose radius of winding is large. Moreover, his method of replacing the tedious summations for wires of finite sections by comparing them with the corresponding values for thin strips is somewhat empirical and not quite satisfying. $\mathrm{He}$ justified his method by numerical application to the case of a singlelayer solenoid wound with thin strips of no radial thickness and no insulation space; that is, to a continuous cylindrical current sheet. This is the only case where an exact formula existed which could be used as a check. With a radius of winding of $25 \mathrm{~cm}$, the results agreed with the current-sheet formula to 1 part in $1,000,000$, and Rosa, therefore, concluded that there is no reason why it should not be equally exact in the case of round wires with insulation spaces between them. Since he does not start with an exact formula and then neglect infinitesimals of known order, it is difficult to say just what is the percentage error involved in Rosa's method due to the curvature of the windings and their spacings, as well as the axial component of current. In view of the increasing precision of electrical measurements and on account of certain absolute measurements which are being undertaken at this bureau, it seemed worth while again to consider this problem with the object of obtaining a more exact formula or one in which the percentage error could be estimated.

In this paper the wire is regarded as a bundle of helical filaments, all having the same pitch $2 \pi p$ and same axial length $l=2 \pi p N$. The $y$ axis being taken as the axis of the cylinder, the initial section of the wire $S$, in which the current enters, being in the $x y$ plane, then two points $P_{1}\left(x_{1} y_{1}\right)$ and $P_{2}\left(x_{2} y_{2}\right)$ in this initial section characterize the

1 Rosa, Calculation of the Self-Inductance of Single-Layer Coils, Bull. of the Bureau of Standards, $\boldsymbol{Z}$, No. 2. 
two helical current filaments passing through these points. The current density is assumed to be in the direction of the helical filament, of magnitude $u\left(x_{1} y_{1}\right)$ for $P_{1}$ and $u\left(x_{2} y_{2}\right)$ for $P_{2}$. Beginning with the Newtonian integrals for the vector potential, it is found that the inductance of the helical distribution $L_{\mathrm{h}}$ (apart from the lead wires) is

$$
L_{\mathrm{n}}=\mathcal{S} \mathcal{S} u\left(x_{1} y_{1}\right) d S_{1} \mathcal{S} \mathcal{S} u\left(x_{2} y_{2}\right) M\left(x_{1} y_{1} x_{2} y_{2}\right) d S_{2}
$$

where the integration extends twice over the initial wire section and the function $M\left(x_{1} y_{1} x_{2} y_{2}\right)$ is a symmetrical function of the two points $P_{1}$ and $P_{2}$, which may be called the mutual inductance of the two filaments passing through these points. It is defined by a definite integral. By means of Neumann's theorem, this is expressed as a Fourier-Bessel expansion whose coefficients involve definite integrals. After some mathematical analysis, most of which is relegated to the appendix, an approximation (86) for $M$ is obtained which is correct to the second order, inclusive, in the small quantities $\frac{x_{2}-x_{1}}{a}, \frac{y_{2}-y_{1}}{a}$, and $\frac{p}{a}$, where $a$ is the radius of the cylindrical form upon which the wire is wound. This consists of the logarithmic term $-4 \pi a N \log \sqrt{\left(x_{2}-x_{1}\right)^{2}+\left(y_{2}-y_{1}\right)^{2}}$, finite terms, and infinitesimal ones of the order $\frac{p}{a},\left(\frac{p}{a}\right)^{2} \log \frac{p}{a}$, and $\left(\frac{p}{a}\right)^{2}$. The largest terms neglected are of the order of $\left(\frac{p}{a}\right)^{3} \log \frac{p}{a}$. It is then an easy matter to integrate this expression and obtain the inductance of a helical distribution of current in a wire of any ordinary shape. The current density is not assumed to be uniform, but its value is expanded by Maclaurin's theorem, and thus a term arises which takes account of the nonuniform distribution of current and admits of estimating the source of error due to this unavoidable circumstance.

A fairly simple formula (108) is obtained in case the wire section $S$ is symmetrical about the $x$ and $y$ axes through its center of gravity. Special cases of importance are those of circular wire (114) and rectangular wire (131). The computation of inductance of a helix $40 \mathrm{~cm}$ in length, of 400 turns, with a pitch of $1 \mathrm{~mm}$ and diameter of circular wire $1 / 2 \mathrm{~mm}$, having a mean cylindrical radius $a=15 \mathrm{~cm}$, gives a value $L_{\mathrm{L}}=26,553,512+16\left(1+2 \frac{a u_{\mathrm{x}}}{u}\right) \mathrm{cm}$ where $u$ is the current density and $u_{\mathrm{x}}$ its partial derivative in the $x$ direction. In case the current density is uniform $u_{\mathrm{x}}=0$ and the $16 \mathrm{~cm}$ is added to the inductance. In case the current density varies inversely as the distance from the axis (the "natural distribution") $\frac{2 a u_{x}}{u}=-2$ so that 
$16 \mathrm{~cm}$ is subtracted. The two distributions differ by $32 \mathrm{~cm}$ in inductance, or about 1 part in 1,000,000. Rosa's value is less than this by only 4 parts in $1,000,000$.

If the circular wire in this example were replaced by square wire of equal thickness, the inductance is $L_{\mathrm{h}}=26,543,066+21\left(1+\frac{2 a u_{\mathrm{x}}}{u}\right)$, a decrease of 1 part in 2,600 from the circular case.

In Appendix 10 the exact expressions are obtained for the magnetic field of a helical current filament, infinite in length. An equation is obtained for the magnetic lines of force in the immediate neighborhood of the helix in a plane through its axis. The lines of magnetic force very close to the filament (and in this plane) are identical with the equipotential lines of a charged grating, which are shown in Figure 13 of volume 1 of Maxwell's Treatise.

In Appendix 11 the mutual inductance of the leads with the helix is computed for a certain arrangement of the former.

\section{GEOMETRICAL CONSIDERATIONS}

If the point $P_{1}\left(x_{1} y_{1} O\right)$ lies in that part of the $x y$ plane between the planes $y=0$ and $y=2 \pi p$ for which $x$ is positive, then it has the rectangular coordinates $x=x_{1}, y=y_{1}, z=0$ where $0<y_{1}<2 \pi p$. It has the cylindrical coordinates $r=x_{1} \theta=0, y=y_{1}$. If this point (fig. 1) be given a uniform angular velocity, $\frac{d \theta}{d t}$ (either positive or negative) about the $y$ axis combined with a simultaneous linear velocity, $p \frac{d \theta}{d t}$, in the $y$ direction, it will trace a helical space curve extending from $y=-\infty$ when $\theta=-\infty$ to $y=+\infty$ when $\theta=+\infty$ which lies upon the cylinder $r=x_{1}=$ const. The rectangular coordinates of any point $P$ on this helix are (fig. 1)

$$
\left.\begin{array}{l}
x=r \cos \theta \\
z=-r \sin \theta \\
y=y_{1}+p \theta
\end{array}\right\}
$$

If the angle $\theta$ be allowed to have any real magnitude and if the pitch $p$ be the same for all helices in space, then the position of any point in space may be uniquely specified by the three coordinates $r, \theta$, and $y$, the $r$ and $\theta$ fixing, respectively, the cylinder and the plane through the $y$ axis upon which the point lies, and the coordinate $y$, specifying the particular helix of all those lying upon the cylinder $r=$ constant which passes through the given point this coordinate $y$ being defined as the $y$ coordinate of the point on the first positive turn of the helix corresponding to $\theta=0$ ( $y$ must always lie between zero and $2 \pi p$ ).

A conducting wire of any section, which is wound upon the cylinder $r=a$ to form a single-layer solenoid, is frequently regarded as approxi- 
mately equivalent to $N$ circular turns, each turn being similar to its neighbor but displaced a distance $2 \pi p$ from it in the $y$ direction. A much more accurate representation of the wire is to consider it as the volume generated by imparting to the first section of the wire in the $x y$ plane the above-mentioned screw motion consisting of a uniform angular velocity $\frac{d \theta}{d t}$ about the $y$ axis combined with a simultaneous linear velocity $p \frac{d \theta}{d t}$ in the $y$ direction. (See fig. 1.) Thus all sections of the wire by the plane $\theta=$ constant have the same shape. By $P(r \theta y)$ we shall designate any point in this section, $\theta=$ constant,

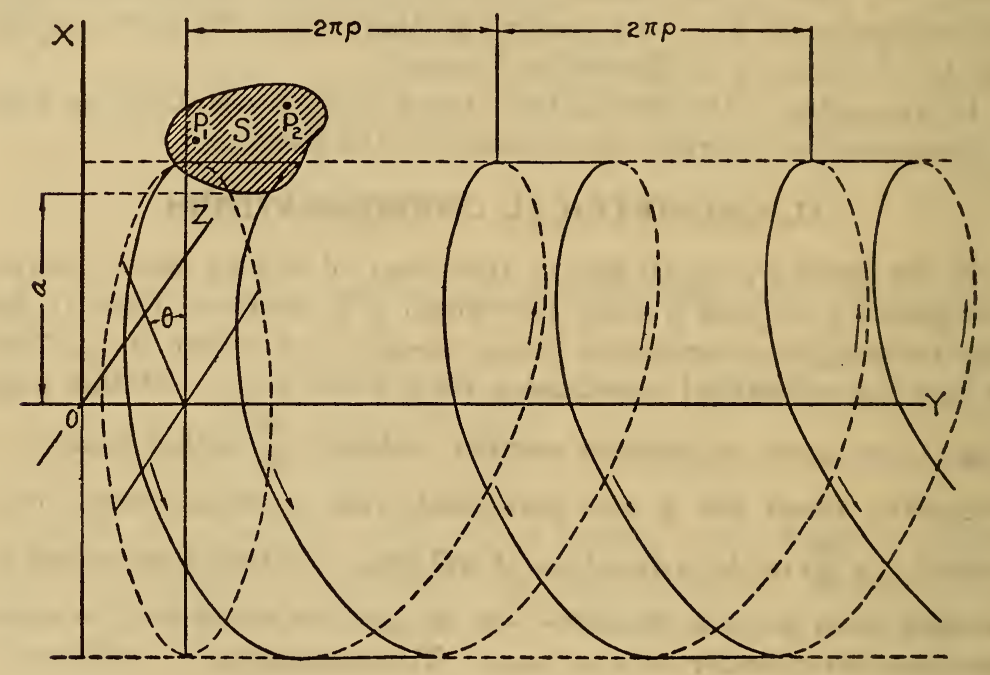

FIG. 1.-Section of helical winding

which lies on the helical filament which passes through the initial point $P(r, O, y)$ in the initial section. As $\theta$ changes from $\theta$ to $\theta+d \theta$ this point $P$ traces the element of arc

$$
d s=\sqrt{r^{2}+p^{2}} d \theta
$$

Similarly, any element of area $d S=d r d y$ of this section generates the volume element

$$
d v=d r d y r d \theta
$$

The latter is independent of the pitch $2 \pi p$. It thus follows that the volume of a turn of the helicoidal wire is the same as that of the corresponding circular wire for which $p=0$. In fact, the helical turn could be produced by cutting the circular turn by the plane $\theta=0$ and giving it a uniform shear, which leaves the volume unchanged because the length of each infinitesimal circular filament of the circular turn 
is increased by this shear in the same ratio that its normal cross section is diminished.

Since the cross section of the conductor is not actually zero, we may regard the wire as a bundle of helices each having the infinitesimal cross section $d r d y$ in the plane $\theta=$ constant. However, the plane $\theta=$ constant is not a normal plane for the helical curve. The tangent to this curve has the direction cosines $l_{\mathrm{h}}, m_{\mathrm{h}}, n_{\mathrm{h}}$. Since $r$ and $y$ remain constant as the point moves along a given helix while $\theta$ alone varies, it follows from (1) and (2) since $l_{\mathrm{h}}=\frac{d x}{d s}$

$$
\left.\begin{array}{l}
l_{\mathrm{h}}=\frac{-\sin \theta}{\sqrt{1+\frac{p^{2}}{r^{2}}}} \\
m_{\mathrm{h}}=\frac{\mathrm{p}}{r \sqrt{1+\frac{p^{2}}{r^{2}}}} \\
n_{\mathrm{h}}=\frac{-\cos \theta}{\sqrt{1+\frac{p^{2}}{r^{2}}}}
\end{array}\right\}
$$

The normal to the section $\theta=$ constant has the direction cosines

$$
\left.\begin{array}{l}
l_{\mathrm{n}}=-\sin \theta \\
m_{\mathrm{n}}=0 \\
n_{\mathrm{n}}=-\cos \theta
\end{array}\right\}
$$

\section{DISTRIBUTION OF STEADY CURRENT}

It $w$ ill be assumed that the (vector) current density $j$ at any point in the section $\theta=$ constant has the direction of the helix which passes through this point; that is, the direction of the above-mentioned screw motion. The direction cosines of $j$ are, therefore, given by (4). Thus $j$ has the same magnitude at any point $P(r \theta y)$ of the conducting section $\theta=$ constant as it has on the initial point of the same helix at $P(r, \cap, y)$ in the plane $\theta=0$. The magnitude of $j$ will be denoted by $u(r y)$. If the wire carries the total current unity, then

$$
1=\int d r \int d y u(r y)\left\{l_{\mathrm{n}} l_{\mathrm{h}}+m_{\mathrm{n}} m_{\mathrm{h}}+n_{\mathrm{n}} n_{\mathrm{h}}\right\}
$$

or by (4) and (5)

$$
1=\int d x \int d y \sqrt{\underline{1+\frac{(x y)}{r^{2}}}}
$$


This integration may be taken over the plane section of the wire $\theta=0$, the $x y$ plane where the current enters the helix.

The rectangular and the cylindrical components of the current density at a point $(r \theta y)$ in the wire are given by

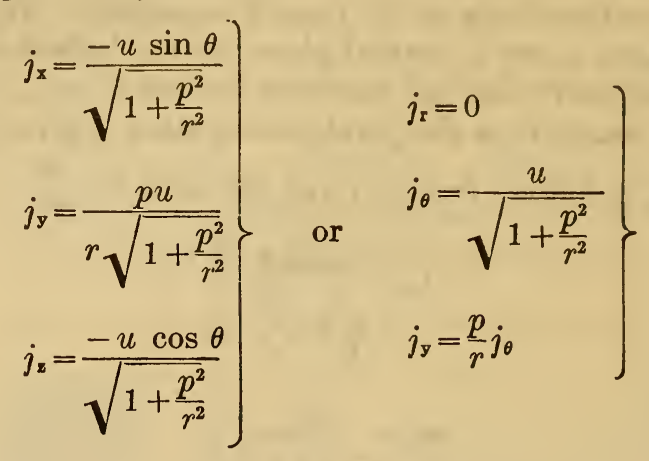

Whatever the function, $u=u(r, y)$, the current density $j$ is solenoidal; that is, the divergence of $j$ is zero. The distribution of steady current over the section of the wire must remain a matter of assumption, the exact nature of which must depend upon experimental evidence. Two of the simplest cases are: $(a)$ The magnitude of the current density $u(\mathrm{ry})$ is inversely proportional to the length of the helical filament; that is, $u=\frac{c}{\sqrt{r^{2}+p^{2}}}$. In this case the line integral of the electric field taken along a line of flow from the section $\theta=\theta_{1}$ to $\theta=\theta_{2}$ has the same value for all the current filaments in the section. This may be called the natural distribution. (b) The current density is uniform over the section; that is, $u(r y)=$ constant $=c$. The value of $c$ in either case is determined by equation (6) when the shape of the section of the conducting wire is known.

\section{FUNDAMENTAL ELECTRICAL EQUATIONS}

The magnetic vector $H$ due to any finite distribution of current is solenoidal and may be derived from a vector potential $A$ by the relation

$$
H=\operatorname{curl} A
$$

In the case of steady current, the fundamental equation of the electromagnetic field is

which requires that

$$
\operatorname{curl} H=4 \pi j
$$

$$
\operatorname{div} j=0
$$

The last equation requires that all currents flow in closed circuits. It is evident that from (8) and (9) $A$ must satisfy the equation

$$
\operatorname{curl}^{2} A \equiv-\nabla^{2} A+\nabla \operatorname{div} A=4 \pi j
$$


which is not sufficient to uniquely determine $A$. However, it may be shown that if $j$ represents a finite, solenoidal current distribution the Maxwell equation (9), together with the condition that $H$ is continuous and vanishes at infinity, does uniquely determine the vector $H$. Consequently, all the solutions of (11) which are continuous with their first derivatives and vanish at infinity must have everywhere the same curl and, therefore, lead to the same value of $H$. Thus if the vector $A_{1}$ satisfies (11) one may form the new vector $A_{2}=A_{1}+\nabla \phi$ where $\phi$ is a scaler function. Then $\operatorname{curl} A_{1}=\operatorname{curl} A_{2}$, which shows that both vectors $A_{1}$ and $A_{2}$ give the same magnetic field. Moreover, $\operatorname{curl}^{2} A_{1}=\operatorname{curl}^{2} A_{2}=4 \pi j$, which shows that $A_{2}$ is also a solution of (11).

Since any solution of (11) will serve the purpose, it is customary to choose for simplicity that one which is solenoidal, so that

$$
\begin{gathered}
\nabla^{2} A=-4 \pi j \\
\operatorname{div} A=0
\end{gathered}
$$

subject to the condition that $A$ and its first derivative is continuous and vanishes canonically at infinity like the Newtonian potential function. The solution of (12) is the (vector) Newtonian potential

$$
A=\iiint \frac{j\left(x^{1} y^{1} z^{1}\right) d x^{1} d y^{1} d z^{1}}{\sqrt{\left(x-x^{1}\right)^{2}+\left(y-y^{1}\right)^{2}+\left(z-z^{1}\right)^{2}}}
$$

which will also satisfy (13) if $j$ satisfies (10). The integral in (14) is taken over the volume of all conductors where $j$ is different from zero. The elementary contribution to the value of this integral of a current $I$ flowing in the (vector) element $\vec{d} s$ is

$$
\frac{I \overrightarrow{d s}}{\sqrt{\left(x-x^{1}\right)^{2}+\left(y-y^{1}\right)^{2}+\left(z-z^{1}\right)^{2}}}
$$

and this leads (by taking the curl according to 8) to the Biot-Savart law for the magnetic field due to a current element. As long as electric currents are regarded as closed, the truth or falsity of this law must remain beyond the possibility of experimental test and this even if one admits for the moment that the conception of the magnetic field is not a mathematical abstraction but is capable of direct experimental evaluation. For it has long been known that this law could be modified by the addition of terms of the type $\frac{\partial \psi}{\partial s}$, where $\psi$ is any single-valued point function, without altering the value of the magnetic field due to a closed current. Therefore, if one applies the integral (14) to a part of a circuit which is not closed (as for example 
the $N$ turns of the helix), this implies that the value of $A$ for the lead-in wires and other apparatus by which the circuit is to be closed will be computed according to the same (Newtonian) integral. With this understanding the value of the vector potential $A^{\mathrm{h}}$, due to the current in the helicoidal wire at any point in space $P_{2}$ whose rectangular coordinates are $x_{2}, y_{2}, z_{2}$, or whose helical coordinates are $r_{2}, \theta_{2}, y_{2}$, where $\theta_{2}$ may have any real value, is given by the vector equation

$$
A^{\mathrm{h}}=\int r_{1} d r_{1} \int d y_{1} \int_{0}^{2 \pi \mathrm{N}} \frac{j\left(r_{1} y_{1}\right) d \theta_{1}}{\sqrt{R^{2}+\left[y_{2}-y_{1}+p\left(\theta_{2}-\theta_{1}\right)\right]^{2}}}
$$

where the integration of $r_{1}$ and $y_{1}$ is over the initial wire section $S$, and where

$$
R^{2}=r_{1}^{2}+r_{2}^{2}-2 r_{1} r_{2} \cos \left(\theta_{2}-\theta_{1}\right)
$$

The vector equation (15) may be replaced by its three rectangular components by substituting for the vector $j$ its three rectangular components in succession. These are given by (7). If we then project the $x$ and $z$ components of $A^{\mathrm{h}}$ along the directions of increasing $r$ and $\theta$ according to the equations

we obtain

$$
\begin{aligned}
& A_{\mathrm{r}}^{\mathrm{h}}=A_{\mathrm{x}}^{\mathrm{h}} \cos \theta_{2}-A_{\mathrm{z}}^{\mathrm{h}} \sin \theta_{2} \\
& A_{\theta}^{\mathrm{h}}=-A_{\mathrm{z}}^{\mathrm{h}} \sin \theta_{2}-A_{\mathrm{z}}{ }^{\mathrm{h}} \cos \theta_{2}
\end{aligned}
$$

$$
\begin{aligned}
& A_{\theta}^{\mathrm{h}}=\int \frac{r_{1} d r_{1}}{\sqrt{1+\frac{p^{2}}{r_{1}^{2}}}} \int u\left(r_{1} y_{1}\right) d y_{1} \int_{0}^{2 \pi \mathrm{N}} \frac{\cos \left(\theta_{2}-\theta_{1}\right) d \theta_{1}}{\sqrt{R^{2}+\left[y_{2}-y_{1}+p\left(\theta_{2}-\theta_{1}\right)\right]^{2}}} \\
& A_{\mathbf{r}}{ }^{\mathrm{h}}=\int \frac{r_{1} d r_{1}}{\sqrt{1+\frac{p^{2}}{r_{1}^{2}}}} \int u\left(r_{1} y_{1}\right) d y_{1} \int_{0}^{2 \pi \mathrm{N}} \frac{\sin \left(\theta_{2}-\theta_{1}\right) d \theta_{1}}{\sqrt{R^{2}+\left[y_{2}-y_{1}+p\left(\theta_{2}-\theta_{1}\right)\right]^{2}}} \\
& A_{\mathbf{y}}{ }^{\mathrm{h}}=\int \frac{r_{1} d r_{1}}{\sqrt{1+\frac{p^{2}}{r_{1}^{2}}}} \int u\left(r_{1} y_{1}\right) d y_{1} \int_{0}^{2 \pi \mathrm{N}} \frac{p / r_{1} d \theta_{1}}{\sqrt{R^{2}+\left[y_{2}-y_{1}+p\left(\theta_{2}-\theta_{1}\right)\right]^{2}}}
\end{aligned}
$$

where the integration with respect to $r_{1}$ and $y_{1}$ is taken orer the initial section $S$ of the wire in the plane $\theta=0$ (or $z=0$ ), or over any section of wire $\theta=$ constant.

It is possible to evaluate the integrals (18) for $A^{\mathrm{h}}$ at any point in space in infinite series, involving cylinder functions and hypergeometric functions. To write out these series in general would be unnecessarily tedious, principally because of the enumeration of cases necessary according to the position of the point $P_{2}\left(r_{2} \theta_{2} y_{2}\right)$. Howerer, if we are concerned only with the self-inductance of the helix or its own mutual electromagnetic forces, we require merely a knowledge of the 
vector potential $A$ at points inside the conducting wire. For the inductive or electromagnetic action of this helix upon other conductors (such as the lead-in wires or other coils), it will usually bo satisfactory to consider the current to be continuously distributed over the entire winding space of the coil. If the other conductors are not too close to the helix, this assumption will satisfy the most precise requirements of experiments, for one may show that the periodic variations or "ripples" in the magnetic field, due to the discrete regular spacing of the windings, fall off exponentially and very rapidly as the point moves away from the conducting wires.

In order that we may restrict the analysis of the magnetic field (or vector potential) to those regions where we actually need it, we may at this point consider the definition and general formulation of the inductance of the helix.

\section{DEFINITION OF INDUCTANCE OF THE HELIX}

The circuit must be closed through the helix by means of lead-in wires and other apparatus. Let $A^{1}$ be the vector potential due to the current in these leads, etc. Then $A=A^{1}+A^{\mathrm{b}}$ represents the potential due to this compound circuit which is closed and carries unit current. The electromagnetic energy $T$ of this current distribution is

$$
T=\frac{1}{2} L(1)^{2}=\frac{L}{2}=\frac{1}{8 \pi} \iiint H^{2} d x d y d z=\frac{1}{2} \iiint(j \cdot A) d x d y d z
$$

The last integral is taken only over the volume of the conductors and is derived by classical transformations from the first, together with the field equations and boundary conditions. From this equation the inductance of the circuit may be written (since $(j \cdot A)$ represents scalar product) as

$$
L=\mathcal{S} \mathcal{S} \mathcal{S}\left(j_{\mathrm{h}} \cdot A^{\mathrm{b}}\right) d v_{\mathrm{h}}+\underset{\int}{\int} \mathcal{S} \mathcal{S} \mathcal{S}\left(j_{\mathrm{h}} \cdot A^{\mathrm{l}}\right) d A_{\mathrm{h}}+\mathcal{S} \mathcal{S} \mathcal{S}\left(j_{1} \cdot A^{\mathrm{b}}\right) d v_{1}+
$$

where $d v_{\mathrm{h}}$ is the volume element of the helix, $d v_{1}$ that of the leads. If we make use of the Newtonian vector potentials in each case

where

$$
A^{\mathrm{h}}=\iiint \frac{j_{\mathrm{h}}{ }^{\prime} d v_{\mathrm{h}}{ }^{\prime}}{D} \quad A^{1}=\iiint \frac{j_{1}{ }^{\prime} d v_{1}{ }^{\prime}}{D}
$$

this becomes

$$
D^{2}=\left(x-x^{\prime}\right)^{2}+\left(y-y^{\prime}\right)^{2}+\left(z-z^{\prime}\right)^{2}
$$

$$
\begin{aligned}
L= & \int_{\mathrm{h}} \int_{\mathrm{h}^{\prime}} \frac{\left(j_{\mathrm{h}} j_{\mathrm{h}}{ }^{\prime}\right) d v_{\mathrm{h}} d v_{\mathrm{h}}{ }^{\prime}}{D}+\int_{\mathrm{h}^{\prime}} \int_{1} \frac{\left(j_{\mathrm{h}}{ }^{\prime} \cdot j_{\mathrm{l}}\right) d v_{\mathrm{h}}{ }^{\prime} d v_{1}}{D}+ \\
& \int_{1} \int_{1^{\prime}} \frac{\left(j_{1} \cdot j_{\mathrm{l}}\right) d v_{\mathrm{l}} d v_{1}^{\prime}}{D}+\int_{\mathrm{h}} \int_{1^{\prime}} \frac{\left(j_{\mathrm{h}} \cdot j_{\mathrm{1}}\right) d v_{\mathrm{h}} d v_{\mathrm{l}}^{\prime}}{D}
\end{aligned}
$$


Or

$$
L=L_{\mathrm{h}}+L_{1}+2 M_{\mathrm{lh}}=\text { inductance of the circuit }
$$

where

$$
\begin{aligned}
L_{\mathrm{h}} & =\int_{\mathrm{h}} \int_{\mathrm{h}} \frac{\left(j_{\mathrm{h}} \cdot j_{\mathrm{h}}{ }^{\prime}\right) d v_{\mathrm{h}} d v_{\mathrm{h}}{ }^{\prime}}{D} \\
L_{\mathrm{l}} & =\int_{1} \int_{1} \frac{\left(j_{1} j_{1}{ }^{\prime}\right) d v_{1} \cdot d v_{1}{ }^{\prime}}{D} \\
M_{1 \mathrm{~h}} & =\int_{\mathrm{h}} \int_{1} \frac{\left(j_{\mathrm{h}} \cdot j_{\mathrm{1}}{ }^{\prime}\right) d v_{\mathrm{h}} d v_{1}{ }^{\prime}}{D}=\int_{1}\left(j_{1} \cdot A^{\mathrm{h}}\right) d v_{1}=\int_{\mathrm{h}}\left(j_{\mathrm{h}} \cdot A^{1}\right) \mathrm{d} v_{\mathrm{h}}
\end{aligned}
$$

The quantities $L_{\mathrm{h}}$ and $L_{1}$ may be called the self-inductance of the current distribution of the helix and of the leads, respectively, while $M_{\mathrm{h} 1}$ is their mutual inductance, the assumption being that all are computed by the same type of vector potential, which in this case is the Newtonian one, and which is equivalent to the Biot-Savart law of magnetic force. The two methods here offered of computing $M_{\mathrm{hl}}$ are in reality identical, since they differ only in the order of integrations. One must integrate over the volume of the leads the value of $A^{b}$ at all points in the leads, due to the current in the helix. If the leads are not too close to the helix one may compute $A^{\mathrm{h}}$ as if the current in the helix were distributed in a continuous manner over the winding space of the latter.

We are concerned here primarily with $L_{\mathrm{h}}$, which is characteristic of the helical distribution of current. The foregoing definition of $L_{\mathrm{h}}$ leads by the use of the expressions (18) for $A^{\mathrm{h}}$ to the formula

$$
L_{\mathbf{b}}=\mathcal{S} \mathcal{S} d S_{\mathbf{2}} u\left(r_{2} y_{2}\right) \mathcal{S} \mathcal{S} d S_{1} u\left(r_{1} y_{1}\right) M\left(r_{1} y_{1} r_{2} y_{2}\right)
$$

where

$$
\begin{gathered}
\frac{r_{1} r_{2}}{\sqrt{\left(1+\frac{p^{2}}{r_{1}{ }^{2}}\right)\left(1+\frac{p^{2}}{r_{2}{ }^{2}}\right)}} \int_{0}^{2 \pi \mathrm{N}} d r_{1} \int_{0}^{2 \pi \mathrm{N}} d \theta_{2} \frac{\left.\left(\cos r_{2} y_{2}-\theta_{1}\right)+\frac{p^{2}}{r_{1} r_{2}}\right)}{\sqrt{R^{2}+\left[y_{2}-y_{1}+p\left(\theta_{2}-\theta_{1}\right)\right]^{2}}} \\
R^{2}=r_{1}{ }^{2}+r_{2}{ }^{2}-2 r_{1} r_{2} \cos \left(\theta_{2}-\theta_{1}\right)
\end{gathered}
$$

The function $M\left(r_{1} y_{1} r_{2} y_{2}\right)$ is unaltered by interchange of the subscripts ${ }_{1}$ and ${ }_{2}$ on either variable. The equation (19) exhibits $L_{\mathrm{h}}$ as the surface integral over the section of the wire at $\theta=0$, with respect to the variables $r_{1} y_{1}$, of the surface integral with respect to $r_{2} y_{2}$ of a symmetrical function of the two points $r_{1} y_{1}$ and $r_{2} y_{2}$ in this section. It is evident that $M$, therefore, plays the part of the mutual inductance of two helical filaments each carrying unit current, having the 
same pitch $2 \pi p$ and number of turns $N$, the two filaments being characterized by the points $r_{1} y_{1}$ and $r_{2} y_{2}$ of the original section through which they pass. The magnitude of the current density $u(r y)$ in formula (19) is subject to the restriction of equation (6) which states that the total current through any section of the wire is unity. No further restriction need be made at this point.

Since neither the shape of the wire section nor the nature of the current distribution $u$ enters into the determination of the function $M$, but only the two helical filaments through the points $P_{1}$ and $P_{2}$, respectively, of the initial section $S$, it seems worth while to devote considerable attention to the evaluation of this function in general. We shall obtain an approximate expression for $M$ which is correct to the second order inclusive in the small quantity $\frac{p}{a}$. The ratio of the linear dimensions of the cross section of the wire to the radius $a$ of the cylinder must in all cases be as small as $\frac{2 \pi p}{a}$, and in exceptional cases may be much smaller.

It may be noted that the term $\frac{p^{2}}{r_{1} r_{2}}$ occurring in the numerator of the integral in (20) represents the contribution of the $y$ component of current. The denominator of this integral will approach zero when the points $P_{1}\left(r_{1} y_{1}\right)$ and $P_{2}\left(r_{2} y_{2}\right)$ approach each other, and it is necessary to determine the precise manner in which the function $M$ then becomes infinite.

\section{THE MUTUAL INDUCTANCE $M$ OF TWO HELICAL FILA- MENTS}

If we let $\theta_{2}-\theta_{1}=\phi^{1}$ in (20), it becomes

$$
M=\frac{r_{1} r_{2}}{\sqrt{\left(1+\frac{p^{2}}{r_{1}^{2}}\right)\left(1+\frac{p^{2}}{r_{2}^{2}}\right)}} \int_{0}^{2 \pi \mathrm{N}} d \theta_{1} \int_{-\theta_{1}}^{-\theta_{1}+2 \pi \mathrm{N}} \frac{\cos \phi^{1}+\frac{p^{2}}{r_{1} r_{2}}}{\sqrt{R^{2}+\left(y_{2}-y_{1}+p \phi^{1}\right)^{2}}}
$$

If we integrate first with respect to $\theta_{1}$ with the proper change of limits, this gives

$$
\begin{gathered}
M=\frac{r_{1} r_{2}}{\sqrt{\left(1+\frac{p^{2}}{r_{1}^{2}}\right)\left(1+\frac{p^{2}}{r_{2}^{2}}\right)}}\left\{\int_{-2 \pi \mathrm{N}}^{0} d \phi^{1} \frac{\left(2 \pi N+\phi^{1}\right)\left(\cos \phi^{1}+\frac{p^{2}}{r_{1} r_{2}}\right)}{\sqrt{R^{2}+\left(y_{2}-y_{1}+p \phi^{1}\right)^{2}}}\right. \\
\left.+\int_{0}^{2 \pi N} d \phi^{1} \frac{\left(2 \pi N-\phi^{1}\right)\left(\cos \phi^{1}+\frac{p^{2}}{r_{1} r_{2}}\right)}{\sqrt{R^{2}+\left(y_{2}-y_{1}+p \phi^{1}\right)^{2}}}\right\}
\end{gathered}
$$


If in the first integral we let $\phi=2 \pi N+\phi^{1}$ and in the second let $\phi=2 \pi N-\phi^{1}$ this becomes

$$
\begin{aligned}
& M=\frac{r_{1} r_{2}}{\sqrt{\left(1+\frac{p^{2}}{r_{1}{ }^{2}}\right)\left(1+\frac{p^{2}}{r_{2}^{2}}\right)}} \iint_{0}^{2 \pi N} \phi\left(\cos \phi+\frac{p^{2}}{r_{1} r_{2}}\right) d \phi\left[\frac{1}{\sqrt{R^{2}+\left(l+y_{2}-y_{1}-p \phi\right)^{2}}}\right. \\
& \left.+\frac{1}{\sqrt{R^{2}+\left(l-y_{2}+y_{1}-p \phi\right)^{2}}}\right]=\frac{r_{1} r_{2}}{\sqrt{\left(1+\frac{p^{2}}{r_{1}^{2}}\right)\left(1+\frac{p^{2}}{r_{2}^{2}}\right)}} \iint_{0}^{\frac{l+\mathrm{y}}{\phi}} \frac{\left(\cos \phi+\frac{p^{2}}{r_{1} r_{2}}\right) d \phi}{\sqrt{(l+y-p \phi)^{2}+R^{2}}} \\
& \left.+\int_{0}^{\frac{l-\mathrm{y}}{\mathrm{p}}} \frac{\phi\left(\cos \phi+\frac{p^{2}}{r_{1} r_{2}}\right) d \phi}{\sqrt{\left(\overline{l-y-p \phi)^{2}+R^{2}}\right.}-2 \int_{0}^{\frac{\mathrm{y}}{\mathrm{p}}} \frac{\left(\cos \phi+\frac{p^{2}}{r_{1} r_{2}}\right) d \phi}{\sqrt{(y-p \phi)^{2}+R^{2}}}}\right\}
\end{aligned}
$$

where

$$
y=y_{2}-y_{1} \text { and } l=2 \pi p N
$$

It will be assumed in the following that $y$ and $r_{2}-r_{1}$ are positive. The expansions obtained for $M$ under this restriction may then be made general by interchanging in them $y_{1}$ and $y_{2}$ when $y_{1}>y_{2}$ and by interchanging $r_{1}$ and $r_{2}$ when $r_{1}>r_{2}$ since (22) shows that the value of $M$ is unaffected by interchanging either pair $r_{1}, r_{2}$ or $y_{1}, y_{2}$.

If $\lambda$ is positive, it is known that ${ }^{2}$

$$
\frac{1}{\sqrt{\lambda^{2}+R^{2}}}=\int_{0}^{\infty} d s e^{-\lambda s} J_{0}(s R)
$$

Also by Neumann's addition formula ${ }^{2}$

$$
J_{0}(s R)=2 \sum_{n=0}^{\infty} \epsilon_{\mathrm{n}} J_{\mathrm{n}}\left(r_{1} s\right) J_{\mathrm{n}}\left(r_{2} s\right) \cos n \phi
$$

where $\epsilon_{n}=1$ if $n \Varangle 0$ and $\epsilon_{0}=\frac{1}{2}$ and where $J_{n}$ is a Bessel's function. Combining (24) and (25) gives (if $\lambda$ is positive)

$$
\begin{aligned}
& \frac{1}{\sqrt{\lambda^{2}+R^{2}}}=\frac{1}{\sqrt{\lambda^{2}+r_{1}{ }^{2}+r_{2}{ }^{2}-2 r_{1} r_{2} \cos \phi}} \\
& =2 \int_{0}^{\infty} d s e^{-\lambda s} \sum_{n=0}^{\infty} \epsilon_{n} J_{n}\left(r_{1} s\right) J_{n}\left(r_{2} s\right) \cos n \phi
\end{aligned}
$$

\footnotetext{
${ }^{2}$ N. Nielsen, Handbuch der Theorie der Cylinderfunktionen, pp. 186 and 280.
} 
Replacing $\lambda$ by $y-p \phi$ in (26) one obtains

$$
\begin{gathered}
\int_{0}^{\frac{y}{p}} \frac{\phi\left(\cos \phi+\frac{p^{2}}{r_{1} r_{2}}\right) d \phi}{\sqrt{(y-p \phi)^{2}+r_{1}^{2}+r_{2}{ }^{2}-2 r_{1} r_{2} \cos \phi}} \\
=2 \int_{0}^{\frac{y}{p}} \phi\left(\cos \phi+\frac{p^{2}}{r_{1} r_{2}}\right) d \phi \int_{0}^{\infty} d s e^{-(y-p))} \sum_{n=0}^{\infty} \epsilon_{\mathrm{n}} J_{\mathrm{n}}\left(r_{1} s\right) J_{\mathrm{n}}\left(r_{2} s\right) \cos n \phi \\
=\sum_{n=0}^{\infty} \epsilon_{\mathrm{n}} \int_{0}^{\frac{y}{p}} d \phi \cdot \phi\left[\cos (n-1) \dot{\phi}+\cos (n+1) \phi+\frac{2 p^{2}}{r_{1} r_{2}} \cos n \phi\right] . \\
\cdot \int_{0}^{\infty} d s e^{-(y-p \phi) s} J_{\mathrm{n}}\left(r_{1} s\right) J_{\mathrm{n}}\left(r_{2} s\right) \\
=\frac{1}{p^{2}} \int_{0}^{y} d \lambda(y-\lambda) \sum_{n=0}^{\infty} \epsilon_{\mathrm{n}} \cos \frac{n(y-\lambda)}{p} \int_{0}^{\infty} d s e^{-\lambda_{s} s}\left\{J_{\mathrm{n}-1}\left(r_{1} s\right) J_{\mathrm{n}-1}\left(r_{2} s\right)\right. \\
\left.+J_{\mathrm{n}+1}\left(r_{1} s\right) J_{\mathrm{n}+1}\left(r_{2} s\right)+\frac{2 p^{2}}{r_{1} r_{2}} J_{\mathrm{n}}\left(r_{1} s\right) J_{\mathrm{n}}\left(r_{2} s\right)\right\}
\end{gathered}
$$

Hence (22) may be written

$$
M\left(r_{2} y_{2} r_{1} y_{1}\right)=\frac{2}{p^{2} \sqrt{\left(1+\frac{p^{2}}{r_{1}^{2}}\right)\left(1+\frac{p^{2}}{r_{2}^{2}}\right)}}\left\{\frac{F(l+y)+F(l-y)}{2}-F(y)\right\}
$$

where

$$
\begin{aligned}
& F(y)=r_{1} r_{2} p^{2} \int_{0}^{\frac{y}{p}} \frac{\phi\left(\cos \phi+\frac{p^{2}}{r_{1} r_{2}}\right) d \phi}{\sqrt{(y-p \phi)^{2}+\left(r_{2}-r_{1}\right)^{2}+4 r_{1} r_{2} \sin ^{2} \frac{\phi}{2}}} \\
& =r_{1} r_{2} \int_{0}^{b} d \lambda .(y-\lambda) \int_{0}^{\infty} d s e^{-\lambda s}\left\{\bar{J}_{1}\left(r_{1} s\right) J_{1}\left(r_{2} s\right)+\frac{p^{2}}{r_{1} r_{2}} J_{0}\left(r_{1} s\right) J_{0}\left(r_{2} s\right)\right\} \\
& +r_{1} r_{2} \sum_{n=1}^{\infty} \int_{0}^{\infty} d s\left\{\frac{y e^{\frac{i n g}{p}}}{s+\frac{i n}{p}}-\frac{e^{\frac{i n g}{p}}}{\left(s+\frac{i n}{p}\right)^{2}}+\frac{e^{-s y}}{\left(s+\frac{i n}{p}\right)^{2}}\right\} \text {. } \\
& \cdot\left\{J_{\mathrm{n}-1}\left(r_{1} s\right) J_{\mathrm{n}-1}\left(r_{2} s\right)+J_{\mathrm{n}+1}\left(r_{1} s\right) J_{\mathrm{n}+1}\left(r_{2} s\right)+\frac{2 p^{2}}{r_{1} r_{2}} J_{\mathrm{n}}\left(r_{1} s\right) J_{\mathrm{n}}\left(r_{2} s\right)\right\}
\end{aligned}
$$

where the real part of this complex quantity is to be taken. Similarly, the substitution of $l_{ \pm} y$ for $y$ in this expression gives $F\left(l_{ \pm} y\right)$. It is assumed in these definitions (29) that $y \equiv y_{2}-y_{1}>0$ and $r_{2}-r_{1}>0$. $102333^{\circ}-26 \dagger-2$ 
By taking the real parts of these expressions, the formula (28) may be written

$$
\begin{aligned}
M=\frac{2}{p^{2} \sqrt{\left(1+\frac{p^{2}}{r_{1}^{2}}\right)\left(1+\frac{p^{2}}{r_{2}^{2}}\right)}}\left\{G_{0}(y)-\frac{G_{0}(l+y)+G_{0}(l-y)}{2}\right. \\
+G_{1}(y)-\frac{G_{1}(l+y)+G_{1}(l-y)}{2} \\
\left.+(l-y) G_{2}+G_{3}\right\}
\end{aligned}
$$

where

$$
\left.\begin{array}{c}
G_{0}(y)=-r_{1} r_{2} \int_{0}^{\nabla} d \lambda_{1} \int_{0}^{\lambda_{1}} d \lambda \int_{0}^{\infty} d s e^{-\lambda s}\left\{J_{1}\left(r_{1} s\right) J_{1}\left(r_{2} s\right)+\frac{p^{2}}{r_{1} r_{2}} J_{0}\left(r_{1} s\right) J_{0}\left(r_{2} s\right)\right\}(31) \\
G_{1}(y)=r_{1} r_{2} p^{2} \sum_{n=1}^{\infty} \frac{1}{n^{2}} \int_{0}^{\infty} d s \frac{1-\frac{p^{2} s^{2}}{n^{2}}}{\left(1+\frac{p^{2} s^{2}}{n^{2}}\right)^{2}} e^{-\mathrm{y} s}\left\{J_{\mathrm{n}-1}\left(r_{1} s\right) J_{\mathrm{n}-1}\left(r_{2} s\right)\right\} \\
\left.+J_{\mathrm{n}+1}\left(r_{1} s\right) J_{\mathrm{n}+1}\left(r_{2} s\right)+\frac{2 p^{2}}{r_{1} r_{2}} J_{\mathrm{n}}\left(r_{1} s\right) J_{\mathrm{n}}\left(r_{2} s\right)\right\} \\
\quad G_{2}=r_{1} r_{2} \sum_{n=1}^{\infty} \cos \frac{n y}{p} \int_{0}^{\infty} \frac{s d s}{s^{2}+\frac{n^{2}}{p^{2}}}\left\{J_{\mathrm{n}-1}\left(r_{1} s\right) J_{\mathrm{n}-1}\left(r_{2} s\right)\right\} \\
\left.+J_{\mathrm{n}+1}\left(r_{1} s\right) J_{\mathrm{n}+1}\left(r_{2} s\right)+\frac{2 p^{2}}{r_{1} r_{2}} J_{\mathrm{n}}\left(r_{1} s\right) J_{\mathrm{n}}\left(r_{2} s\right)\right\} \\
G_{3}=r_{1} r_{2} \sum_{n=1}^{\infty} \sin \frac{n y}{p} \int_{0}^{\infty} d s \frac{2 n s}{p\left(s^{2}+\frac{n^{2}}{p^{2}}\right)^{2}}\left\{J_{\mathrm{n}-1}\left(r_{1} s\right) J_{\mathrm{n}-1}\left(r_{2} s\right)\right. \\
\left.\quad+J_{\mathrm{n}+1}\left(r_{1} s\right) J_{\mathrm{n}+1}\left(r_{2} s\right)+\frac{2 p^{2}}{r_{1} r_{2}} J_{\mathrm{n}}\left(r_{1} s\right) J_{\mathrm{n}}\left(r_{2} s\right)\right\}
\end{array}\right\}
$$

In the following section we shall take up the simplification of these $G$ functions, carrying the approximations to the second order inclusire in the small quantities $\frac{p}{a}, \frac{r_{2}-r_{1}}{a}$, and $\frac{y}{a}\left(=\frac{y_{2}-y_{1}}{a}\right)$. Where necessary, it will be assumed that $r_{1}<r_{2}$. Since $M$ and each $G$ function is unaltered by interchange of $r_{1}$ and $r_{2}$, the result of any expansion which is expressed in terms of $r_{1}$ and $r_{2}$ under the assumption that $r_{2}-r_{1}>0$, will be valid when $r_{1}-r_{2}<0$ if $r_{1}$ and $r_{2}$ are interchanged. Similarly, for $y_{2}-y_{1}$. 


\section{EVALUATION OF $M\left(r_{1} y_{1} r_{2} y_{2}\right)$}

\section{PRINCIPAL TERMS ARISING FROM $G_{0}$}

Since $y / l$ is small

$$
G_{0}(y)-\frac{G_{0}(l+y)+G_{0}(l-y)}{2}=G_{0}(y)-G_{0}(l)-\frac{y^{2}}{2} G_{0}^{\prime \prime}(l)
$$

where by $(31)-\frac{y^{2}}{2} G_{0}{ }^{\prime \prime}(l)$ may be replaced by

$$
-\frac{y^{2} G_{0}{ }^{\prime \prime}(l)}{2}=\frac{a y^{2}}{2} \int_{0}^{\infty} d t e^{\frac{-l t}{8}} J_{1}{ }^{2}(t)=\frac{y^{2} a}{2} \phi_{1}(k)
$$

where

$$
k^{2}=\frac{1}{1+\left(\frac{l}{2 a}\right)^{2}} \text { so that } \frac{l}{a}=\frac{2 \sqrt{1-k^{2}}}{k}
$$

and

$$
\phi_{\mathrm{n}}(\mu)=\int_{0}^{\infty} e^{-2 t \sqrt{\frac{1}{\mu^{3}}-1}} J_{\mathbf{n}}^{2}(t) d t
$$

As considerable use will be made of the functions $\phi_{n}(\mu)$, some of their properties may be investigated. For this purpose we may make use of a special case of (26), replacing $\lambda$ by $2 \lambda$ and letting $r_{1}=r_{2}=1$ and $\theta_{1}=0$. This gives

Or if

$$
\frac{1}{2 \sqrt{\lambda^{2}+\sin ^{2} \frac{\theta}{2}}}=\int_{0}^{\infty} d t e^{-2 \lambda t}\left\{J_{0}^{2}(t)+2 \sum_{n=1}^{\infty} J_{\mathbf{n}}{ }^{2}(t) \cos n \theta\right\}
$$

$$
\left.\begin{array}{rl}
\frac{\mu^{2}}{} & =\frac{1}{1+\lambda^{2}} \\
\frac{\mu}{4 \sqrt{1-\mu^{2} \cos ^{2} \frac{\theta}{2}}} & =\int_{0}^{\infty} d t e^{-2 t \sqrt{\frac{1}{\mu^{2}}-1}}\left\{\frac{J_{0}^{2}(t)}{2}+\sum_{n=1}^{\infty} J_{n}^{2}(t) \cos n \theta\right\} \\
& =\frac{1}{2} \phi_{0}(\mu)+\sum_{n=1}^{\infty} \phi_{\mathrm{n}}(\mu) \cos n \theta
\end{array}\right\}
$$

according to the definition (38) of $\phi_{n}(\mu)$. The identity (40) shows that $\phi_{\mathrm{n}}(\mu)$ is the Fourier coefficient in the cosine development of the periodic function of $\theta$,

$$
\frac{\mu}{4 \sqrt{1-\mu^{2} \cos ^{2} \frac{\theta}{2}}} \text {. This gives a second integral }
$$

representation of $\phi_{\mathrm{n}}(\mu)$, namely,

$$
\phi_{\mathrm{n}}(\mu)=\frac{\mu}{\pi} \int_{0}^{\frac{\pi}{2}} \frac{\cos 2 n \theta d \theta}{\sqrt{1-\mu^{2} \cos ^{2} \theta}}=\frac{(-1)^{\mathrm{n}} \mu}{\pi} \int_{0}^{\frac{\pi}{2}} \frac{\cos 2 n \theta d \theta}{\sqrt{1-\mu^{2} \sin ^{2} \theta}}
$$


It follows from this integral form of $\phi_{\mathrm{n}}(\mu)$ or from (38) by taking account of the differential equation of the Bessels function, that $\phi_{\mathrm{n}}(\mu)$ satisfies the second order differential equation.

$$
\mu^{3} \frac{d}{d \mu}\left[\frac{1-\mu^{2}}{\mu^{\prime}} \frac{d \phi_{\mathrm{n}}}{d \mu}\right]=4\left(n-\frac{1}{2}\right)\left(n+\frac{1}{2}\right) \phi_{\mathrm{n}}
$$

and the identical relation

$$
\left(n+\frac{3}{2}\right) \phi_{\mathrm{n}+2}(\mu)=2\left(\frac{2}{\mu^{2}}-1\right)(n+1) \phi_{\mathrm{n}+1}(\mu)-\left(n+\frac{1}{2}\right) \phi_{\mathrm{n}}(\mu)
$$

By expanding the integrand of (38) in a power series, it is found that

$$
\phi_{\mathrm{n}}(\mu)=\frac{\Gamma\left(n+\frac{1}{2}\right)}{\sqrt{\pi} \Gamma(n+1)}\left(\frac{\mu}{2}\right)^{2 \mathrm{n}+1} F\left(n+\frac{1}{2}, n+\frac{1}{2}, 2 n+1, \mu^{2}\right)
$$

where $F$ is the hypergeometric function. For the particular cases $n=0$ and $n=1$ (44) shows that

$$
\begin{gathered}
\phi_{0}(\mu)=\frac{\mu}{\pi} K(\mu) \\
\phi_{1}(\mu)=\frac{\mu}{\pi}\left\{\left(\frac{2}{\mu^{2}}-1\right) K(\mu)-\frac{2}{\mu^{2}} E(\mu)\right\}
\end{gathered}
$$

where $K(\mu)$ and $E(\mu)$ are the complete elliptic integrals of the first and second kind, respectively, whose modulus is $\mu$. Another useful relation derivable from (42) and (43) is

$$
\begin{gathered}
\frac{1-\mu^{2}}{\mu} \frac{d}{d \mu} \phi_{\mathrm{n}}(\mu)=\left(n-\frac{1}{2}\right)\left\{\phi_{\mathrm{n}-1}(\mu)-\left(\frac{2}{\mu^{2}}-1\right) \phi_{\mathrm{n}}(\mu)\right\} \\
=\left(n+\frac{1}{2}\right)\left\{-\phi_{\mathrm{n}+1}(\mu)+\left(\frac{2}{\mu^{2}}-1\right) \phi_{\mathrm{n}}(\mu)\right\}
\end{gathered}
$$

which may also be put in the forms

and

$$
\int \frac{(n+1) \phi_{\mathrm{n}+1}(\mu)-n \phi_{\mathrm{n}}(\mu)}{\mu^{2} \sqrt{1-\mu^{2}}} d \mu=-\frac{\sqrt{1-\mu^{2}}}{2 \mu}\left\{\phi_{\mathrm{n}+1}(\mu)+\phi_{\mathrm{n}}(\mu)\right\}
$$

$$
\int \frac{\phi_{\mathrm{n}+1}(\mu)-\phi_{\mathrm{n}}(\mu)}{\mu\left(1-\mu^{2}\right)} d \mu=-\frac{1}{2\left(n+\frac{1}{2}\right)}\left\{\phi_{\mathrm{n}+1}(\mu)+\phi_{\mathrm{n}}(\mu)\right\}
$$

By combining (47) and (42) one finds also

$$
\begin{gathered}
\int \frac{\phi_{\mathrm{n}}(\mu) d \mu}{\mu^{3}}=\frac{1}{4\left(n+\frac{1}{2}\right)}\left\{\phi_{\mathrm{n}-1}(\mu)-\left(\frac{2}{\mu^{2}}-1\right) \phi_{\mathrm{n}}(\mu)\right\}=\frac{1}{4 n^{2}-1} \cdot \frac{1-\mu^{2}}{\mu} \cdot \frac{d \phi_{\mathrm{n}}}{d \mu}= \\
=\frac{1}{4\left(n-\frac{1}{2}\right)}\left\{-\phi_{\mathrm{n}+1}(\mu)+\left(\frac{2}{\mu^{2}}-1\right) \phi_{\mathrm{n}}(\mu)\right\}
\end{gathered}
$$


The special cases of (48) and (49) where $n=0$ and $n=1$, together with the use of (43) and (47), give the following indefinite integrals:

$$
\begin{gathered}
\int \frac{\phi_{0}(\mu)}{\mu^{3}} d \mu=-\frac{E(\mu)}{\pi \mu} \\
\int \frac{\phi_{1}(\mu)}{\mu^{3}} d \mu=\frac{1}{3 \pi \mu^{3}}\left\{\left(2-\mu^{2}\right) E(\mu)-2\left(1-\mu^{2}\right) K(\mu)\right\} \\
\int \frac{\phi_{1}(\mu) d \mu}{\mu^{2} \sqrt{1-\mu^{2}}}=\frac{\sqrt{1-\mu^{2}}}{\pi \mu^{2}}\{E(\mu)-K(\mu)\}
\end{gathered}
$$

In general, $\mu$ is less than one but it is necessary to know the manner in which $\phi_{\mathrm{n}}(\mu)$ becomes infinite when $\mu$ approaches one. This is shown by the form of the hypergeometric function given in equations (22) and (23) of Appendix 1. This shows that $\phi_{\mathrm{n}}(\mu)$ may also be put in the form

$$
\phi_{\mathrm{n}}(\mu)=\frac{\mu^{2 \mathrm{n}+1}}{2 \pi}\left\{u_{\mathrm{n}}\left(1-\mu^{2}\right)-v_{\mathrm{n}}\left(1-\mu^{2}\right) \log \left(1-\mu^{2}\right)\right\}
$$

where

$$
\left.\begin{array}{c}
u_{\mathrm{n}}\left(1-\mu^{2}\right)=\frac{1}{\left[\Gamma\left(n+\frac{1}{2}\right)\right]^{2}} \sum_{s=0}^{\infty}\left(1-\mu^{2}\right)^{\mathrm{s}}\left[\frac{\Gamma\left(s+n+\frac{1}{2}\right)}{\Gamma(s+1)}\right]^{2} T_{\mathrm{s}}(n) \\
v_{\mathrm{n}}\left(1-\mu^{2}\right)=F\left(n+\frac{1}{2}, n+\frac{1}{2}, 1,1-\mu^{2}\right) \\
T_{\mathrm{s}}(n) \equiv 2 \sum_{t=s}^{\infty}\left(\frac{1}{t+n+\frac{1}{2}}-\frac{1}{t+1}\right)
\end{array}\right\}
$$

so that

$$
\begin{gathered}
T_{0}(0)=4 \log 2, T_{0}(n)=4 \log 2-2 \sum_{t=0}^{n-1} \frac{1}{t+\frac{1}{2}} \text { if } n>0 \\
T_{s}(n)=T_{0}(n)+2 \sum_{t=0}^{s-1}\left(\frac{1}{t+1}-\frac{1}{t+n+\frac{1}{2}}\right) \ldots \ldots \text { if } s>0
\end{gathered}
$$

In particular, if $1-\mu^{2}$ is infinitesimal, then neglecting infinitesimals of higher order than $1-\mu^{2}$

$$
\left.\begin{array}{rl}
\phi_{\mathrm{n}}(\mu)= & \frac{1}{2 \pi}\left\{T_{0}(n)-\log \left(1-\mu^{2}\right)-\left(n^{2}-\frac{1}{4}\right)\left(1-\mu^{2}\right) \log \left(1-\mu^{2}\right)\right. \\
+ & \left.\left(n+\begin{array}{c}
1 \\
2
\end{array}\right)\left[\left(n+\begin{array}{c}
1 \\
2
\end{array}\right) T_{1}(n)-T_{0}(n)\right]\left(1-\mu^{2}\right) \ldots\right\} \\
\frac{1-\mu^{2}}{\mu} \frac{d \phi_{\mathrm{n}}(\mu)}{d \mu}=\frac{1}{\pi}\left\{1+\left(n^{2}-\frac{1}{4}\right)\left(1-\mu^{2}\right) \log \left(1-\mu^{2}\right)+\left(n+\frac{1}{2}\right) \cdot\right. \\
\left.\left[n-\frac{1}{2}+T_{0}(n)-\left(n+\frac{1}{2}\right) T_{1}(n)\right]\left(1-\mu^{2}\right)\right\}
\end{array}\right\}
$$


Another relation which will be found useful is derivable from the Fourier's series (40). Let $f(\theta)$ be any function of $\theta$ which is developable in a cosine series as follows:

$$
f(\theta)=\frac{1}{2} f_{0}+\sum_{n=1}^{\infty} f_{n} \cos n \theta \quad 0<\theta<\pi
$$

Multiplying (40) and (57) together and integrating the product with respect to $\theta$ from $O$ to $\pi$ gives

$$
\frac{\mu}{2 \pi} \int_{0}^{\pi} \frac{f(\theta) d \theta}{\sqrt{1-\mu^{2} \cos ^{2} \frac{\theta}{2}}}=\frac{1}{2} f_{0} \phi_{0}(\mu)+\sum_{n=1}^{\infty} f_{\mathrm{n}} \phi_{\mathrm{n}}(\mu)
$$

In particular if $f(\theta)=\left(\frac{\pi^{2}}{3}-\pi \theta+\frac{\theta^{2}}{2}\right) \cos \theta=\sum_{n=1}^{\infty} \frac{\cos (n-1) \theta+\cos (n+1) \theta}{n^{2}}$ this gives for the value of a series which appears later

$$
\begin{gathered}
\sum_{n=1}^{\infty} \frac{\phi_{n-1}(k)+\phi_{n+1}(k)}{n^{2}}=2\left(\frac{k^{2}-2}{\pi k}\right)\left(\frac{\pi^{2}}{12} K(k)-\int_{0}^{\frac{\pi}{2}} \frac{\theta^{2} d \theta}{\sqrt{1-k^{2} \sin ^{2} \theta}}\right) \\
+\frac{4}{\pi k}\left(\frac{\pi^{2}}{12} E(k)-\int_{0}^{\frac{\pi}{2}} \theta^{2} \sqrt{1-k^{2} \sin ^{2} \theta} d \theta\right)
\end{gathered}
$$

The value of the series on the left of (59) may be determined by graphical integration of the right side with less labor than by computations based upon the power series definition of $\phi_{\mathrm{n}}$.

By means of the foregoing properties of the functions $\phi_{n}$, we may proceed with the simplification of the terms $G_{0}(l)$ and $G_{0}(y)$ in (35) defined by (31). Changing $s$ to $\frac{t}{r_{2}}$ in (31) gives

$$
\begin{gathered}
-G_{0}(l)=\sqrt{r_{1} r_{2}} \int_{0}^{l l} d \lambda_{1} \int_{0}^{\lambda_{1}} d \lambda \sqrt{\frac{r_{1}}{r_{2}}} \int_{0}^{\infty} d t e^{-\frac{\lambda t}{r_{2}}} J_{1}(t) J_{1}\left(\begin{array}{c}
r_{1} t \\
r_{2}
\end{array}\right) \\
+\frac{p^{2}}{\sqrt{r_{1} r_{2}}} \int_{0}^{l} d \lambda_{1} \int_{0}^{\lambda_{1}} d \lambda \sqrt{\frac{r_{1}}{r_{2}}} \int_{0}^{\infty} d t e^{-\frac{\lambda t}{r_{2}}} J_{0}(t) J_{0}\left(\frac{r_{1} t}{r_{2}}\right)
\end{gathered}
$$

The function of the two variables $\frac{\lambda}{r_{2}}$ and $\frac{r_{1}}{r_{2}}$ defined by the integral

$$
\sqrt{\frac{r_{1}}{r_{2}}} \int_{0}^{\infty} d t e^{-\frac{\lambda t}{r_{3}}} J_{\mathrm{n}}(t) J_{\mathrm{n}}\left(\frac{r_{1} t}{r_{2}}\right)
$$

becomes infinite when $\frac{\lambda}{r_{2}}$ and $\frac{r_{1}}{r_{2}}$ simultaneously approach the limits 0 and 1 , respectively. It may be studied by noting that this function satisfies a certain partial differential equation in these two 
variables and reduces to $\phi_{\mathrm{n}}\left(\frac{1}{\sqrt{1+\left(\frac{\lambda}{2 r}\right)^{2}}}\right)$ when $\frac{r_{1}}{r_{2}}=1$, while its derivative with respect to $\frac{\lambda}{r_{2}}$ vanishes when $\frac{r_{1}}{r_{2}}=1$. A much simpler discussion is, however, possible, for we may prove that this function of the two variables $\frac{\lambda}{r_{2}}$ and $\frac{r_{1}}{r_{2}}$ may be expressed as a function of the single function $\mu\left(\frac{\lambda}{r_{2}}, \frac{r_{1}}{r_{2}}\right)$ defined by

$$
\mu^{2}=\frac{4\left(\frac{r_{1}}{r_{2}}\right)}{\left(\frac{\lambda}{r_{2}}\right)^{2}+\left(1+\frac{r_{1}}{r_{2}}\right)^{2}}=\frac{1}{1+\frac{\lambda^{2}+\left(r_{2}-r_{1}\right)^{2}}{4 r_{1} r_{2}}}=\frac{4 r_{1} r_{2}}{\lambda^{2}+\left(r_{1}+r_{2}\right)^{2}}
$$

This theorem takes the following form

$$
\sqrt{\frac{r_{1}}{r_{2}}} \int_{0}^{\infty} d t e^{-\frac{\lambda t}{r_{2}}} J_{\mathrm{n}}(t) J_{\mathrm{n}}\left(\frac{r_{1} t}{r_{2}}\right)=\phi_{\mathrm{n}}(\mu)
$$

where $\mu$ is defined by (61). This important relation may be established as follows: Multiplying (26) by $\sqrt{\frac{r_{1} r_{2}}{2}}$ gives

$$
\begin{gathered}
\frac{\sqrt{r_{1} r_{2}}}{2 \sqrt{\lambda^{2}+r_{1}^{2}+r_{2}^{2}-2 r_{1} r_{2} \cos \left(\theta_{2}-\theta_{1}\right)}}=\sqrt{r_{1} r_{2}} \int_{0}^{\infty} d s e^{-\lambda s}\left\{\frac{J_{0}\left(r_{1} s\right) J_{0}\left(r_{2} s\right)}{2}\right. \\
\left.+\sum_{n=1}^{\infty} J_{\mathrm{n}}\left(r_{1} s\right) J_{n}\left(r_{2} s\right) \cos n\left(\theta_{2}-\theta_{1}\right)\right\}
\end{gathered}
$$

or

$$
\begin{aligned}
& \frac{1}{4} \sqrt{\frac{4 r_{1} r_{2}}{\lambda^{2}+\left(r_{1}+r_{2}\right)^{2}}} \frac{1}{\sqrt{1-\frac{4 r_{1} r_{2}}{\lambda^{2}+\left(r_{1}+r_{2}\right)_{2}} \cos ^{2}\left(\frac{\theta_{2}-\theta_{1}}{2}\right)}} \\
& =\sqrt{\frac{r_{1}}{r_{2}}} \int_{0}^{\infty} d t e^{-\frac{\lambda t}{r_{2}}}\left\{\frac{J_{0}(t) J_{0}\left(\frac{r_{1} t}{r_{2}}\right)}{2}+\sum_{n=1}^{\infty} J_{\mathrm{n}}(t) J_{\mathrm{n}}\left(\frac{r_{1} t}{r_{2}}\right) \cos n\left(\theta_{2}-\theta_{1}\right)\right\}
\end{aligned}
$$

If, then, $\mu$ has the value given by (61), this identity takes the form

$$
\begin{gathered}
\frac{\mu}{4 \sqrt{1-\mu^{2} \cos ^{2}\left(\frac{\theta_{2}-\theta_{1}}{2}\right)}}=\sqrt{\frac{r_{1}}{r_{2}}} \int_{0}^{\infty} d t e^{-\frac{\lambda t}{r_{2}}}\left\{\frac{J_{0}(t) J_{0}\left(\frac{r_{1} t}{r_{2}}\right)}{2}\right. \\
\left.+\sum_{n=1}^{\infty} J_{\mathrm{n}}(t) J_{\mathrm{n}}\left(\frac{r_{1} t}{r_{2}}\right) \cos n\left(\theta_{2}-\theta_{1}\right)\right\}
\end{gathered}
$$

A comparison of this Fourier series with the series (40) gives the theorem (62). 
The second integral.in (60) contains the second order factor $p^{2}$. Therefore, we may place $r_{1}=r_{2}=a$ in this term, neglecting higher order infinitesimals than the second. The theorem (62) by the use of (45) then enables one to write (60) in the form

$$
\begin{gathered}
-G_{0}(l)=\sqrt{r_{1} r_{2}} \int_{0}^{l} d \lambda_{1} \int_{0}^{\lambda_{1}} d \lambda \phi_{1}(\mu) \\
+\frac{p^{2}}{\pi a} \int_{0}^{l} d \lambda_{1} \int_{0}^{\lambda_{1}} d \lambda \frac{K\left(\frac{1}{\sqrt{1+\left(\frac{\lambda}{2 a}\right)^{2}}}\right)}{\sqrt{1+\left(\frac{\lambda}{2 a}\right)^{2}}}
\end{gathered}
$$

where $\mu$ and $\lambda$ are connected by (61). The first integral is evaluated in Appendix 3, equation (19). The second may be evaluated graphically. (See Appendix 2.) Letting

$$
\begin{aligned}
x_{1}=r_{1}-a, x_{2}=r_{2}-a, x= & x_{2}-x_{1}, y=y_{2}-y_{1}, z=x+i y=x_{2}-x_{1} \\
& +i\left(y_{2}-y_{1}\right)
\end{aligned}
$$

and combining the results of Appendix 2 and Appendix 3 gives

$$
\begin{gathered}
-G_{0}(l)=\frac{4 a^{3}}{\pi}\left\{A_{0}+\frac{p^{2}}{a^{2}} B_{0}+A_{1}\left(\frac{x_{1}+x_{2}}{a}\right)-\frac{\pi l}{8 a}\left(\frac{x_{2}-x_{1}}{a}\right)-\frac{\pi l}{16 a}\left(\frac{x_{2}{ }^{2}-x_{1}{ }^{2}}{a^{2}}\right)\right. \\
\left.+A_{3} \frac{x_{1} x_{2}}{a^{2}}+A^{4}\left(\frac{x_{2}-x_{1}}{a}\right)^{2}-\frac{\left(x_{2}-x_{1}\right)^{2}}{8 a^{2}} \log \left(\frac{x_{2}-x_{1}}{8 a}\right)\right\}
\end{gathered}
$$

The term in $G_{0}{ }^{\prime \prime}(l)$ in (36) may be written

$$
-\frac{y^{2}}{2} G_{0}^{\prime \prime}(l)=\frac{4 a^{3}}{\pi}\left[\frac{\left(2-k^{2}\right) K(k)-2 E(k)}{8 k}\right] \frac{y^{2}}{a^{2}}
$$

The value of $G_{0}(y)$ is found in Appendix 4, equation (1), as

$$
G_{0}(y)=\frac{4 a^{3}}{\pi}\left\{\frac{\left(x_{2}-x_{1}\right)^{2}}{8 a^{2}} \log \left(\frac{x_{2}-x_{1}}{8 a}\right)+\frac{y^{2}}{16 a^{2}}-\frac{z^{2}}{8 a^{2}} \log \frac{z}{8 a}\right\}_{\text {real part }}
$$

Hence if we let

$$
A_{5}=\frac{1}{8}\left\{\frac{\left(2-k^{2}\right) K(k)-2 E(k)}{k}+\frac{1}{2}\right\}
$$

we may collect all terms arising from $G_{0}$ by adding together (66), (67), and (68) in the form

$$
\begin{gathered}
G_{0}(y)-\frac{G_{0}(l+y)+G_{0}(l-y)}{2} \\
=\frac{4 a^{3}}{\pi}\left\{A_{0}+\frac{p^{2}}{a^{2}} B_{0}+A_{1}\left(\frac{x_{1}+x_{2}}{a}\right)-\frac{\pi l}{8 a}\left(\frac{x_{2}-x_{1}}{a}\right)-\frac{\pi l}{16 a}\left(\frac{x_{2}{ }^{2}-x_{1}{ }^{2}}{a^{2}}\right)\right. \\
\left.+A_{3} \frac{x_{1} x_{2}}{a^{2}}+A_{4}\left(\frac{x_{2}-x_{1}}{a}\right)^{2}+A_{5}\left(\frac{y_{2}-y_{1}}{a}\right)^{2}-\frac{z^{2}}{8 a^{2}} \log \frac{z}{8 a}\right\}
\end{gathered}
$$


where the real part of the last term is to be taken and where it is assumed that $r_{2}-r_{1}>0$; that is, $x_{2}-x_{1}>0$. When $x_{2}-x_{1}<0$ then $x_{1}$ and $x_{2}$ must be interchanged in the above equation. The finite constants $A_{0}, A_{1}, A_{3}$, and $A_{4}$ are functions of $k=\frac{1}{\sqrt{1+\left(\frac{l}{2 a}\right)^{2}}}$ given by (20) of Appendix 3. The constants $B_{0}$ and $A_{5}$ are determined, respectively, by Appendix 2 and by (69).

\section{PRINCIPAL TERMS ARÍSING FROM $G_{1}$}

From the definition (32) since $G_{1}$ contains the second order factor $p^{2}$, it is evident that to the second order inclusive

$$
\begin{aligned}
& -\frac{G_{1}(l+y)+G_{1}(l-y)}{2} \\
& =-G_{1}(l)=-a^{2} p^{2} \sum_{n=1}^{\infty} \frac{1}{n^{2}} \int_{0}^{\infty} d s e^{-l s}\left\{J_{\mathrm{n}-1}{ }^{2}(a s)+J_{\mathrm{n}+1}{ }^{2}(a s)\right\} \\
& =-a p^{2} \sum_{n=1}^{\infty} \frac{1}{n^{2}} \int_{0}^{\infty} d t e^{-\frac{l}{8} t}\left\{J_{\mathrm{n}-1}{ }^{2}(t)+J_{\mathrm{n}+1}{ }^{2}(t)\right\} \\
& =-a^{3} \cdot \frac{p^{2}}{a^{2}} \sum_{n=1}^{\infty} \frac{\phi_{n-1}(k)+\phi_{\mathrm{n}+1}(k)}{n^{2}}
\end{aligned}
$$

by the definition (38). Making use of (59), this gives

$$
-\frac{G_{1}(l+y)+G_{1}(l-y)}{2}=-\frac{4 a^{3}}{\pi} \frac{p^{2}}{a^{2}} B_{1}
$$

where

$$
\begin{gathered}
B_{1}=-\left(\frac{2-k^{2}}{2 k}\right)\left[\frac{\pi^{2}}{12} K(k)-\int_{0}^{\frac{\pi}{2}} \frac{\theta^{2} d \theta}{\sqrt{1-k^{2} \sin ^{2} \theta}}\right]+\frac{1}{k}\left[\frac{\pi^{2}}{12} E(k)\right. \\
\left.-\int_{0}^{\frac{\pi}{2}} \theta^{2} \sqrt{1-k^{2} \sin ^{2}} \theta d \theta\right]=k \int_{0}^{\frac{\pi}{2}} d \theta \frac{\left(\frac{\pi^{2}}{12}-\theta^{2}\right)\left(\frac{1}{2}-\sin ^{2} \theta\right)}{\sqrt{1-k^{2} \sin ^{2} \theta}}
\end{gathered}
$$

The second order term $G_{1}(y)$ is examined in Appendix 5. Equations (13) and (14) of that appendix, together with (71 and (72), enable us to write

$$
\begin{gathered}
G_{1}(y)-\frac{G_{1}(l+y)+G_{1}(l-y)}{2}= \\
=\frac{4 a^{3}}{\pi} \cdot \frac{p^{2}}{a^{2}}\left\{-B_{1}+B_{2}+\frac{\pi^{2}}{12} \log \frac{2 a}{p}-\frac{\pi^{2}}{12} \log \frac{z}{p}-\frac{1}{2} F_{1}\left(\frac{z}{p}\right)\right\}_{\text {real part }}
\end{gathered}
$$


where

$$
B_{2}=\frac{1}{8} \sum_{n=1}^{\infty} \frac{T_{0}(n-1)+T_{0}(n+1)}{\imath^{2}} \text { and }\left\{\begin{array}{l}
T_{0}(o)=4 \log 2 \\
T_{0}(k)=4 \log 2-2 \sum_{t=0}^{\mathrm{k}-1} \frac{1}{t+\frac{1}{2}}
\end{array}\right.
$$

and

$$
F_{1}\left(\frac{z}{p}\right)=\frac{\partial}{\partial p} p \sum_{n=1}^{\infty} \frac{1}{n^{2}} \int_{0}^{\infty} \frac{s e^{\frac{\mathrm{inz}}{p}}}{1+s^{2}} d s
$$

The imaginary part of these expressions is to be discarded.

\section{PRINCIPAL TERMS OF $G_{2}$ AND $G_{3}$}

In equation (11) of Appendix 6 is derived the following expression if $r_{2}-r_{1}>0$

$$
\int_{0}^{\infty} \frac{s}{s^{2}+\frac{n^{2}}{p^{2}}} J_{\mathbf{k}}\left(r_{1} s\right) J_{\mathbf{k}}\left(r_{2} s\right) d s=\frac{p e^{-\mathrm{n}\left(\frac{\mathrm{r}_{1}-\mathrm{r}_{1}}{\mathrm{p}}\right)}}{2 n \sqrt{r_{1} r_{2}}}=\frac{p e^{-\mathrm{n}\left(\frac{\mathrm{x}_{2}-\mathrm{x}_{1}}{\mathrm{p}}\right)}}{2 n \sqrt{r_{1} r_{2}}}
$$

to the second order inclusive in $x_{2}-x_{1}$ and $p$. This gives in (33)

$$
(l-y) G_{2}=\sqrt{r_{1} r_{2}} p(l-y) \sum_{n=1}^{\infty} \frac{e^{-\frac{n x}{p}} \cos \frac{n y}{p}}{n}
$$

Similarly from the result of (13), Appendix 6, namely,

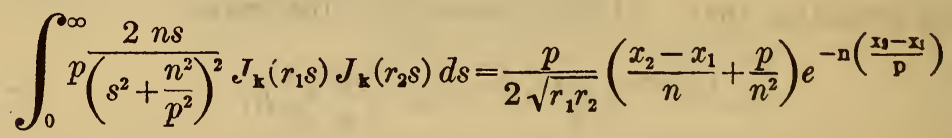

is derived a value for (34)

$$
G_{3}=\sqrt{r_{1} r_{2}} p \sum_{n=1}^{\infty}\left(\frac{x}{n}+\frac{p}{n^{2}}\right) e^{-\frac{n x}{\mathrm{p}}} \sin n \frac{y}{p}
$$

Hence, if we place $\sqrt{r_{1} r_{2}}=a\left(1+\frac{x_{1}+x_{2}}{2 a}\right)$ in the first order terms and place $\sqrt{r_{1} r_{2}}=a$ in the second order term, and in conformity with the previous forms let $z=x+i y=x_{2}-x_{1}+i\left(y_{2}-y_{1}\right)$, we get, if $x \equiv x_{2}-x_{1}$ $>0$ and $y \equiv y_{2}-y_{1}>0$

$$
\begin{aligned}
(l-y) G_{2} & +G_{3}=\frac{4 a^{3}}{\pi}\left\{\frac{p}{a} \cdot \frac{l}{a} \cdot \frac{\pi}{4}\left(1+\frac{x_{1}+x_{2}}{2 a}\right) \sum_{n=1}^{\infty} \frac{e^{-\frac{\mathrm{n} z}{\mathrm{p}}}}{n}\right. \\
& \left.+\frac{p^{2}}{a^{2}} \cdot \frac{\pi}{4} i \sum_{n=1}^{\infty} \frac{1}{n^{2}}\left(1+\frac{n z}{p}\right) e^{-\frac{\mathrm{n} z}{\mathrm{p}}}\right\}
\end{aligned}
$$


where the imaginary component is to be rejected. This may also be written

$$
\begin{gathered}
(l-y) G_{2}+G_{3}= \\
=\frac{4 a^{3}}{\pi} \cdot \frac{p^{2}}{a^{2}}\left\{\frac{\pi}{4}\left[\frac{l}{p}+\frac{l}{2 p}\left(\frac{x_{1}+x_{2}}{a}\right)+\frac{i z}{p}\right] F_{2}\left(\frac{z}{p}\right)+\frac{\pi i}{4} F_{3}\left(\frac{z}{p}\right)\right\}
\end{gathered}
$$

where

$$
\left.\begin{array}{l}
\mathrm{F}_{2}\left(\frac{z}{p}\right)=\sum_{n=1}^{\infty} \frac{e^{-\mathrm{n} \frac{\mathrm{z}}{\mathrm{p}}}}{n}=-\log \left(1-e^{-\frac{\mathrm{z}}{\mathrm{p}}}\right) \\
F_{3}\left(\frac{z}{p}\right)=\sum_{n=1}^{\infty} \frac{e^{-\frac{\mathrm{n} z}{\mathrm{p}}}}{n^{2}}
\end{array}\right\}
$$

\section{COLLECTION OF ALL TERMS IN $M\left(x_{t} y_{t} x_{2} y_{s}\right)$}

The function $u\left(x_{1} y_{1}\right) u\left(x_{2} y_{2}\right) M\left(x_{1} y_{1} x_{2} y_{2}\right)$ must be integrated twice over the initial section of the wire in the plane $\theta=0$ to obtain the inductance of the helical distribution of current by (19), where $u\left(x_{1} y_{1}\right)$ is the magnitude of the current density at a point $x_{1} y_{1}$ of this section. Similarly, $u\left(x_{2} y_{2}\right)$ is its value at any second point $x_{2} y_{2}$ of the same section. For the sake of arriving at a simpler and more familiar notation for performing these integrations over a plane. area, it must be remembered that we have adopted the following change of notation. Instead of representing the distance of the two points $P_{1}$ and $P_{2}$ of the plane (oblique) section of the wire from the axis of the cylinder by $x_{1}$ and $x_{2}$, we now use these letters to designate the distance of $P_{1}$ and $P_{2}$ from the cylinder $r=a$, so that

$$
x_{1}=r_{1}-a \quad x_{2}=r_{2}-a
$$

Adding together the equations (70), (73), and (77) and substituting in (30) gives

$$
\begin{aligned}
M\left(x_{1} y_{1} x_{2} y_{2}\right) & =\frac{a^{2}}{p^{2}} \frac{a}{\sqrt{1+\frac{p^{2}}{r_{1}{ }^{2}}} \sqrt{1+\frac{p^{2}}{r_{2}{ }^{2}}}}\left\{\frac{8 A_{0}}{\pi}+\frac{p^{2}}{a^{2}}\left[\frac{8}{\pi}\left(B_{0}-B_{1}+B_{2}\right)-\frac{2 \pi}{3} \log \pi\right]\right. \\
& +\frac{p}{a} \frac{8 A_{1}}{\pi}\left(\frac{x_{1}+x_{2}}{p}\right)+\frac{\pi p^{2}}{a^{2}}\left[\frac{2}{3}+4\left(\frac{z}{2 \pi p}\right)^{2}\right] \log \frac{a}{p} \\
& +\frac{p^{2}}{a^{2}} \frac{8}{\pi}\left[A_{3} \frac{x_{1} x_{2}}{p^{2}}+A_{4} \frac{\left(x_{2}-x_{1}\right)^{2}}{p^{2}}+A_{5} \frac{\left(y_{2}-y_{1}\right)^{2}}{p^{2}}\right] \\
& +\frac{p^{2}}{a^{2}} 4 \pi N\left[1+\frac{x_{1}+x_{2}}{2 a}\right]\left[F_{2}\left(\frac{z}{p}\right)-\frac{z}{2 p}\right] \\
& +\frac{p^{2}}{a^{2}}\left[4 \pi\left(\frac{z}{2 \pi p}\right)^{2} \log \frac{4}{\pi}-\pi\left(\frac{2}{3}+4\left(\frac{z}{2 \pi p}\right)^{2}\right) \log \frac{z}{2 \pi p}\right. \\
& \left.\left.+2\left(-\frac{2}{\pi} F_{1}\left(\frac{z}{p}\right)+i \frac{z}{p} F_{2}\left(\frac{z}{p}\right)+i F_{3}\left(\frac{z}{p}\right)\right)\right]\right\}
\end{aligned}
$$


where

$$
z=\left|x_{2}-x_{1}\right|+i\left|y_{2}-y_{1}\right|
$$

The terms which are functions of the complex variable $z$ are functions of the relative vector distance from the point $P_{1}\left(x_{1} y_{1}\right)$ to the

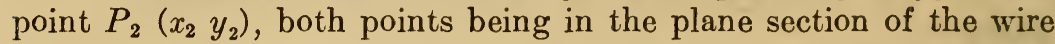
given by $\theta=0$; that is, the plane in which the current enters the helix. The real part of these complex expressions is to be taken. Before taking the real part, it may be noted that these transcendental functions of the complex variable $\frac{z}{p}$ admit of great simplification. In Appendix 7, equation (8), it is shown that

where

$$
\begin{gathered}
F_{2}\left(\frac{z}{p}\right)-\frac{z}{2 p}=-\log \frac{z}{p}+\sum_{n=1}^{\infty} b_{n}\left(\frac{z}{2 \pi p}\right)^{2 \mathrm{n}}=-\log \frac{z}{p}-\sum_{k=1}^{\infty} \log \\
{\left[1+\left(\frac{z}{2 \pi p k}\right)^{2}\right]}
\end{gathered}
$$

$$
b_{n}=\frac{(-1)^{n}}{n} \sum_{k=1}^{\infty} \frac{1}{k^{2 n}}
$$

The series converges provided $|z|<2 \pi p$; that is, provided that $|z|$ or the modulus of $z$ (which is the numerical distance from $P_{1}$ to $P_{2}$ ) shall be less than $2 \pi p$, the distance between successive helical filaments.

In Appendix 8 a number of transformations are made by which it is found-equation (22)-that if $|z|<2 \pi p$

$$
\begin{gathered}
4 \pi\left(\frac{z}{2 \pi p}\right)^{2} \cdot \log \frac{4}{\pi}-\pi\left[\frac{2}{3}+4\left(\frac{z}{2 \pi p}\right)^{2}\right] \log \frac{z}{2 \pi p}+2\left[-\frac{2}{\pi} F_{1}\left(\frac{z}{p}\right)+i \frac{z}{p} F_{2}\left(\frac{z}{p}\right)\right. \\
\left.+i F_{3}\left(\frac{z}{p}\right)\right]=2 \pi\left\{.66267+.63754\left(\frac{z}{2 \pi p}\right)^{2}-2 \sum_{n=2}^{\infty} c_{n}\left(\frac{z}{2 \pi p}\right)^{2 \mathrm{n}}\right\}
\end{gathered}
$$

where

$$
c_{n}=\frac{(-1)^{\mathrm{n}}}{n} \sum_{k=1}^{\infty} \frac{1}{k^{2 n-1}}
$$

Making use of (82) and (84) in (80), we obtain the following formula for the mutual inductance of two unit currents in the two helical filaments having the same pitch $2 \pi p$ and length $l=2 \pi p N$ which pass through the two points $P_{1}$ and $P_{2}$, both in the same plane section of the wire, made by a plane through the axis of the solenoid. 


$$
\begin{aligned}
M\left(x_{1} y_{1} x_{2} y_{2}\right) & =\frac{a}{\sqrt{1+\frac{p^{2}}{r_{1}^{2}}} \sqrt{1+\frac{p^{2}}{r_{2}^{2}}}}\left\{\frac{8 A_{0} a^{2}}{\pi p^{2}}+2 \pi A_{2}+\frac{8 A_{1} a}{\pi p}\left(\frac{x_{1}+x_{2}}{p}\right)\right. \\
+ & \pi\left[\frac{2}{3}+4\left(\frac{z}{2 \pi p}\right)^{2}\right] \log \frac{a}{p}+1.27508 \pi\left(\frac{z}{2 \pi p}\right)^{2} \\
+ & \frac{8}{\pi}\left[A_{3} \frac{x_{1} x_{2}}{p^{2}}+A_{4} \frac{\left(x_{2}-x_{1}\right)^{2}}{p^{2}}+A_{5} \frac{\left(y_{2}-y_{1}\right)^{2}}{p^{2}}\right] \\
& -4 \pi N\left[1+\frac{x_{1}+x_{2}}{2 a}\right] \log \left(\frac{R_{12}}{p}\right) \\
& +4 \pi N\left[1+\frac{x_{1}+x_{2}}{2 a}\right] \sum_{n=1}^{\infty} b_{n}\left(\frac{z}{2 \pi p}\right)^{2 n} \\
& \left.-4 \pi \sum_{n=2}^{\infty} c^{n}\left(\frac{z}{2 \pi p}\right)^{2 n}\right\}
\end{aligned}
$$

In this expression $x_{1}$ is the distance of the point $P_{1}$ from the cylindrical form $(r=a)$ measured in the direction of increasing $r$, where $r$ is the distance from the axis. The coordinate of $P_{1}$ in a direction (in this section of the wire) which is perpendicular to that of $x$ is $y_{1}$. Since $y_{1}$ and $y_{2}$ enter this formula only in the form $y_{2}-y_{1}$, the $x$ axis may be anywhere in the section. The real part of $\log z$ is $\log R_{12}$

$$
R_{12}=\sqrt{\left(x_{2}-x_{1}\right)^{2}+\left(y_{2}-y_{1}\right)^{2}}\left(=\text { the distance from } P_{1} \text { to } P_{2}\right)
$$

The real part of $z$ is to be taken in (86), and in this form we may write without restriction

$$
z=\left(x_{2}-x_{1}\right)+i\left(y_{2}-y_{1}\right) \text { instead of }\left|x_{2}-x_{1}\right|+i\left|y_{2}-y_{1}\right|
$$

because the real part of even powers of $z$ like

$$
z^{2 \mathrm{n}}=\left[x_{2}-x_{1}+i\left(y_{2}-y_{1}\right)\right]^{2 \mathrm{n}}
$$

consists of terms of the type $\left(x_{2}-x_{1}\right)^{2 \mathrm{k}}\left(y_{2}-y_{1}\right)^{2 \mathrm{~s}}$ where $k$ and $s$ are integers. This is unaffected by interchange of $x_{1}$ and $x_{2}$ or of $y_{1}$ and $y_{2}$. Consequently, with the definitions (87) and (88), the expression (86) is perfectly general. It obviously satisfies the requirement that $M$ be a symmetrical function of the two points $P_{1}$ and $P_{2}$.

The numerical coefficients $b_{\mathrm{n}}$ are given by (83); the coefficients $c_{\mathrm{n}}$ by (85). They are also numerical. The coefficient $A_{2}$ is a function of $k=\frac{1}{\sqrt{1+\left(\frac{l}{2 a}\right)^{2}}}$. It also includes all other constant terms which are numerical and is defined by

$$
A_{2}=\frac{4}{\pi^{2}}\left(B_{0}-B_{1}+B_{2}\right)+.66267-\frac{1}{3} \log \pi
$$


where

$$
\begin{aligned}
& B_{0}=\int_{0}^{\eta} d \eta_{2} \int^{\eta_{2}} d \eta_{1} \frac{K\left(\frac{1}{\sqrt{1+\eta_{1}{ }^{2}}}\right)}{\sqrt{1+\eta_{1}{ }^{2}}} ; \eta=\frac{l}{2 a}=\frac{\sqrt{1-k^{2}}}{k} \\
& B_{1}=k \int_{0}^{\frac{\pi}{2}} \frac{\left(\frac{\pi^{2}}{12}-\theta^{2}\right)\left(\frac{1}{2}-\sin ^{2} \theta\right)}{\sqrt{1-k^{2} \sin ^{2} \theta}} d \theta \\
& B_{2}=\frac{1}{8} \sum_{n=1}^{\infty} \frac{T_{0}(n-1)+T_{0}(n+1)}{n^{2}}=-.70 ; \begin{array}{l}
T_{0}(0)=4 \log 2 \\
T_{0}(n)=4 \log 2-2 \sum_{t=0}^{n-1} \frac{1}{t+\frac{1}{2}}
\end{array}
\end{aligned}
$$

so that

$$
\frac{4}{\pi^{2}} B_{2}-\frac{1}{3} \log \pi+.663=.0020 \approx 0
$$

By the use of an integraph, the functions $B_{0}$ and $B_{1}$ have been found, and the function $A_{2}$ computed. (See figs. 2, 3, and 4.) The other coefficients $A_{0}, A_{1}, A_{3}, A_{4}$, and $A_{5}$ are also functions of $k$ given by

$$
\begin{aligned}
& A_{0}=\frac{1}{3}\left\{\frac{\left(1-k^{2}\right) K(k)-\left(1-2 k^{2}\right) E(k)}{k^{3}}-1\right\} \\
& \left.A_{1}=\frac{1}{2} \frac{E(k)}{k}-1\right\} \\
& A_{3}=\frac{2 E(k)-\left(1-k^{2}\right) K(k)}{2 k}-1 \\
& A_{4}=\frac{1}{4}\left\{\frac{2 E(k)-\left(1-\frac{k^{2}}{2}\right) K(k)}{k}-\frac{5}{4}\right\} \\
& A_{5}=\frac{A_{1}}{2}-\underline{A_{6}} \quad \\
& k^{2}=\frac{1}{1+\left(\frac{l}{2 a}\right)^{2}} ; \quad l=2 \pi p N
\end{aligned}
$$

The bracket in the second member of (86) contains terms which, in general, represent four orders of magnitude. When the distance between the points $P_{1}$ and $P_{2}$ is of the same order of magnitude as the pitch of the windings, $\frac{z}{p}$ and $\frac{z}{2 \pi p}$ are finite. In this case the principal term is the finite one $\frac{8 A_{0} a^{2}}{\pi p^{2}}$. The terms nextin importance 
are of first order, which contain either the factor $\frac{a}{p}$ or $4 \pi N$. The second order terms are finite quantities, for we have now multiplied in the second order infinitesimal $\frac{p^{2}}{a^{2}}$. The terms of type $\log \frac{a}{p}$ are of order higher than the first and lower than the second. The expression (86) has been obtained by neglecting terms of the type $\frac{p^{3}}{a^{3}} \log \frac{a}{p}$.

If the points $P_{1}$ and $P_{2}$ approach each other, the two helical filaments approach coincidence throughout their entire length. In this case $\frac{z}{p}$ and $\frac{R_{12}}{p}$ approach zero and the logarithmic term becomes the predominating one, and $M_{12}$ becomes infinite in such a manner that its principal part is $2(2 \pi a N) \log \frac{1}{R_{12}}$. Since $\frac{p}{a}$ is small, the total length of each filament is practically $2 \pi a N$, and this shows that the principal part of the mutual inductance of the two helical filaments, when they are very close together, is the same as if they were straight and parallel.

In taking the real part of the terms of type $z^{2 n}, n=1,2,3 \cdots$ we may place $z=R_{12} e^{i \phi_{12}}$ where $\phi_{12}$ is the angle between the $x$ axis (of this section) and the line $R_{12}$ drawn from $P_{1}$ to $P_{2}$. The real part of $z^{2 \mathrm{n}}$ is $R_{12} \cos n \phi_{12}$, and since the terms in $\sin n \phi_{12}$ are imaginary, and to be discarded, it follows that those remaining $\left(\cos n \phi_{12}\right)$ are unaffected by changing the sign of $\phi_{12}$. These terms thus depend in a simple manner upon the distance $R_{12}$ and its direction. The one term, of course, which is independent of direction is $\log R_{12}$.

\section{GENERAL FORMULA FOR THE INDUCTANCE OF A HELICAL DISTRIBUTION OF CURRENT FOR ANY SHAPE OF SECTION OF THE WIRE}

The inductance of the helical distribution of current has been shown to be

$$
L_{\mathrm{h}}=\iint d x_{2} d y_{2} \iint d x_{1} d y_{1} u\left(x_{1} y_{1}\right) u\left(x_{2} y_{2}\right) M\left(x_{1} y_{1} x_{2} y_{2}\right)
$$

This is a repeated surface integral over the plane section of the wire, first with respect to the point $P_{1}$ and then with respect to $P_{2}$ of the symmetrical function of the two points

$$
u\left(x_{1} y_{1}\right) u\left(x_{2} y_{2}\right) M\left(x_{1} y_{1} x_{2} y_{2}\right)
$$

The vector current density at a point $x y$ of the section has been assumed to have the direction of the helical filament passing through 
this section. Its magnitude is $u(x y)$, and since the vector must be solenoidal and the current is steady the current density has this same value at all points of this helical filament. The magnitude, $u$, may, however, vary in passing from one filament to another; that is, $u=u(x y)$, and there is nothing in electrical theory to indicate the nature of this variation. (This possible nonuniform distribution of steady current is, of course, not to be confused with that which arises from distributed capacity and electromagnetic induction when the current is alternating. The current density in that case would also vary along the length of the same helical filament. The more ambitious problem of determining such a distribution of alternating current to a first approximation might be attacked by the method of integral equations, using (86) as a starting point, which must certainly be correct for vanishing frequency.)

In carrying out an absolute measurement of the highest precision, one must obtain some evidence of an experimental nature as to the function $u(x y)$ or at least show that its variations are negligible for the degree of precision aimed at. There can be no question but that the current density $u$ is practically constant over the section of the wire, but in attempting a second order precision, as in the present formula, one can not avoid a consideration of this nonuniformity. Causes of this variation might be in the nonhomogeneity of the wire caused by drawing or any other internal strains, or in the manner in which the electromotive force is applied to the wire. Two of the simplest assumptions that might be made are $(a)$ that $u$ is constant, or (b) that $u$ varies inversely as the length of the helical filament corresponding to the point $(x y)$; that is, $u=\frac{c}{r}=\frac{c}{a+x}$. This is the socalled "natural distribution." In order to make an estimate of the importance of this effect and to provide a formula by which it may be corrected for, if known, we shall expand $u(x y)$ by Maclaurin's series in terms of the two small variables $x$ and $y$. The degree to which this expansion must be carried, to be commensurate with the other approximations here made, will immediately become evident.

It will be recalled that the formula (97) for the inductance of the helical distribution of current has been obtained on the assumption that the total current flowing through any section of the wire is unity. This places one restriction upon the current density $u(x) y)$ which, since the section is oblique to the helix (and to the flow), has been shown in equation (6) to take the form in the present notation.

$$
\iint \frac{u(x y) d x d y}{\sqrt{1+\frac{p^{2}}{r^{2}}}}=1
$$

This relation shows that all constant terms within the bracket of the 
second member of (86) contribute simply their own value to $L_{\mathrm{n}}$; for the double surface integration indicated by $(97)$ of the terms

$$
\frac{a}{\sqrt{1+\frac{p^{2}}{r_{1}{ }^{2}}} \sqrt{1+\frac{p^{2}}{r_{2}{ }^{2}}}}\left\{\frac{8 A_{0} a^{2}}{\pi p^{2}}+2 \pi A_{2}+\frac{2 \pi}{3} \log \frac{a}{p}\right\}
$$

contributes to $L_{\mathrm{n}}$ the amount

$$
\begin{gathered}
a \int \frac{u\left(x_{2} y_{2}\right) d x_{2} d y_{2}}{\sqrt{1+\frac{p^{2}}{r_{2}^{2}}}} \iint \frac{u_{1}\left(x_{1} y_{1}\right) d x_{1} d y_{1}}{\sqrt{1+\frac{p^{2}}{r_{1}^{2}}}}\left\{\frac{8 A_{0} a^{2}}{\pi p^{2}}+2 \pi A_{2}+\frac{2 \pi}{3} \log \frac{a}{p}\right\} \\
=a\left\{\frac{8 A_{0} a^{2}}{\pi p^{2}}+2 \pi A_{2}+\frac{2 \pi}{3} \log \frac{a}{p}\right\}
\end{gathered}
$$

This is true whatever the current distribution function $u(x y)$.

Furthermore, the remaining terms within the bracket of (86) are all infinitesimal compared to $\frac{8 A_{0} a^{2}}{\pi p^{2}}$, the most important being first order or the order of $\frac{p}{a}$. Since $\left(\frac{p}{a}\right)^{3}$ is negligible, it is evident that, for the remaining integrations, the factor $\frac{1}{\sqrt{1+\frac{p^{2}}{r_{1}{ }^{2}}} \sqrt{1+\frac{p^{2}}{r_{2}{ }^{2}}}}$ outside the bracket may be replaced by 1. For the same reason, it is evident that the Maclaurin expansion

$$
u(x y)=u(0,0)+x\left(\frac{\partial u}{\partial x}\right)_{00}+y\left(\frac{\partial u}{\partial y}\right)_{00}
$$

will be sufficient to obtain a formula correct to the second order, and the first order terms $x\left(\frac{\partial u}{\partial x}\right)_{00}$ and $y\left(\frac{\partial u}{\partial y}\right)_{00}$ need be retained only in combination with the first order terms within the bracket (86).

If for brevity we represent from here on $u(0,0),\left(\frac{\partial u}{\partial x}\right)_{00}$ and $\left(\frac{\partial u}{\partial y}\right)_{00}$ by $u, u_{\mathbf{x}}$, and $u_{\mathbf{y}}$, respectively, the Maclaurin expansion takes the form

$$
u(x y)=u\left\{1+x \frac{u_{\mathbf{x}}}{u}+y \frac{u_{\mathrm{y}}}{u}\right\}
$$

Substituting this form in (98) gives to the first order inclusive

$$
u=\frac{1}{S}\left\{1-\bar{x} \frac{u_{\mathrm{x}}}{u}-\bar{y} \frac{u_{\mathrm{y}}}{u}\right\}
$$

$102333^{\circ}-26 \dagger-3$ 
where $\mathrm{S}$ is the area of the plane section and $\bar{x}$ and $\bar{y}$ the coordinates of its center of gravity. This with (100) gives

$$
\begin{gathered}
u\left(x_{1} y_{1}\right)=\frac{1}{S}\left\{1+\left(x_{1}-\bar{x}\right) \frac{u_{\mathrm{x}}}{u}+\left(y_{1}-\bar{y}\right) \frac{u_{\mathrm{y}}}{u}\right\} \\
u\left(x_{1} y_{1}\right) u\left(x_{2} y_{2}\right)=\frac{1}{S^{2}}\left\{1-2 \bar{x} \frac{u_{\mathrm{x}}}{u}-2 \bar{y} \frac{u_{\mathrm{y}}}{u}+\left(x_{1}+x_{2}\right) \frac{u_{\mathrm{x}}}{u}+\left(y_{1}+y_{2}\right) \frac{u_{\mathrm{y}}}{u}\right\}
\end{gathered}
$$

If in (97) we make use of the expressions (86), (99), and (103) we obtain the general formula

$$
\begin{aligned}
L_{\mathrm{n}} & =a\left\{\frac{8 A_{0} a^{2}}{\pi p^{2}}+2 \pi A_{2}+\frac{2 \pi}{3} \log \frac{a}{p}\right\}-4 \pi a N\left(1+\frac{\bar{x}}{a}\right) \log \frac{1}{p} \\
& +\frac{16 a A_{1}}{\pi}\left(\frac{a}{p}\right)\left(\frac{\bar{x}}{p}\right)+\frac{16 a A_{1}}{\pi}\left[\frac{a u_{\mathrm{x}}}{u}\left(\frac{k_{\mathrm{x}}{ }^{2}-\bar{x}^{2}}{p^{2}}\right)+\frac{a u_{\mathrm{y}}}{u}\left(\frac{k_{\mathrm{xy}}{ }^{2}-\bar{x} \bar{y}}{p^{2}}\right)\right] \\
& +\frac{8 a A_{3}}{\pi}\left(\frac{\bar{x}}{p}\right)^{2}+\frac{16 a A_{4}}{\pi}\left(\frac{k_{\mathrm{x}}{ }^{2}-\bar{x}^{2}}{p^{2}}\right)+\frac{16 a}{\pi} A_{5}\left(\frac{k_{\mathrm{y}}{ }^{2}-\bar{y}^{2}}{p^{2}}\right) \\
& +\pi a\left[1.27508+4 \log \frac{a}{p}\right] \frac{1}{S^{2}} \iint d S_{2} \iint d S_{1}\left(\frac{z}{2 \pi p}\right)^{2} \\
& -4 \pi a N\left[1-2 \bar{x} \frac{u_{\mathrm{x}}}{u}-2 \bar{y} \frac{u_{\mathrm{y}}}{u}\right] \frac{1}{S^{2}} \iint d S_{2} \iint d S_{1} \log R_{12} \\
& -4 \pi a N \frac{1}{S^{2}} \iint d S_{2} \iint d S_{1}\left[\left(\frac{1}{a}+\frac{2 u_{\mathrm{x}}}{u}\right) x_{1}+\frac{2 u_{\mathrm{y}}}{u} y_{1}\right] \log R_{12} \\
& +4 \pi a N\left[1-2 \bar{x} \frac{u_{\mathrm{x}}}{u}-2 \bar{y} \frac{u_{\mathrm{y}}}{u}\right] \frac{1}{S^{2}} \iint d S_{2} \iint d S_{1} \sum_{n=1}^{\infty} b_{\mathrm{n}}\left(\frac{z}{2 \pi p}\right)^{2 \mathrm{n}} \\
& +4 \pi a N \frac{1}{S^{2}} \iint d S_{2} \iint d S_{1}\left[\left(\frac{1}{a}+\frac{2 u_{\mathrm{x}}}{u}\right) x_{1}+\frac{2 u_{\mathrm{y}}}{u} y_{1}\right] \sum_{n=1}^{\infty} b_{\mathrm{n}}\left(\frac{z}{2 \pi p}\right)^{2 \mathrm{n}} \\
& -\frac{4 \pi a}{S^{2}} \iint d S_{2} \iint d S_{1} \sum_{n=2}^{\infty} c_{\mathrm{n}}\left(\frac{z}{2 \pi p}\right)^{2 \mathrm{n}}
\end{aligned}
$$

where $\bar{x}$ and $\bar{y}$ are the coordinates of the center of gravity of the section, $k_{\mathrm{x}}{ }^{2}$ and $k_{\mathrm{y}}{ }^{2}$ the radii of gyration of the section about the $y$ and $x$ axes, respectively, and $S k_{\mathrm{xy}}{ }^{2}$ the product of inertia of the section referred to, a system of axes in which the $y$ axis is a generating line of the cylindrical form. These quantities are giren by the formulas

$$
\left.\begin{array}{c}
\bar{x}=\frac{1}{S} \iint x d S \quad \bar{y}=\frac{1}{S} \iint y d S \quad k_{\mathrm{x}}{ }^{2}=\frac{1}{S} \iint x^{2} d S \\
k_{\mathrm{y}}{ }^{2}=\frac{1}{S} \iint y^{2} d S \quad k_{\mathrm{zy}}{ }^{2}=\frac{1}{S} \iint x y d S
\end{array}\right\}
$$

The real part only of the complex quantity $z=x_{2}-x_{1}+i\left(y_{2}-y_{1}\right)$ is to be taken. 
The formula (104) becomes more simple if referred to a new system of axes parallel to the former, which pass through the center of gravity of the section $(\bar{x}, \bar{y})$. If $S k_{x^{2}}, S k_{y^{2}}$, and $S k_{x y}$ now denote the moments and product of inertia referred to the new axes through the center of gravity, then

$$
k_{x^{2}}=k_{x}{ }^{2}-\bar{x}^{2}, k_{y^{2}}=k_{y}{ }^{2}-\bar{y}^{2}, k_{x y}=k_{x y}{ }^{2}-\overline{x y}
$$

The remaining integrals are also simplified by this transformation so that we may write the general formula as follows:

$$
\begin{aligned}
L_{\mathrm{n}} & =\frac{S a}{\pi}\left[A_{0} \frac{a^{2}}{p^{2}}+2 A_{1} \frac{a \bar{x}}{p^{2}}+A_{3} \frac{\bar{x}^{2}}{p^{2}}\right]+\frac{2 \pi a}{3}\left[1+12 \frac{k_{x^{2}}-k_{y^{2}}}{(2 \pi p)^{2}}\right] \log \frac{a}{p} \\
& +2 \pi a A_{2}+64 \pi a A_{1}\left[\left(\frac{1}{2}+\frac{a u_{\mathrm{x}}}{u}\right) \frac{k_{\mathrm{x}^{2}}}{(2 \pi p)^{2}}+\frac{a u_{\mathrm{y}}}{u} \frac{k_{\mathrm{xy}}}{(2 \pi p)^{2}}\right] \\
& +64 \pi a A_{5}\left[\frac{k_{\mathrm{y}^{2}}-k_{\mathrm{x}^{2}}}{(2 \pi p)^{2}}\right]-4 \pi a N\left(1+\frac{\bar{x}}{a}\right) \log \frac{1}{p}+2 \pi a(1.27508) \frac{k_{\mathrm{x}^{2}}-k_{\mathrm{y}^{2}}}{(2 \pi p)^{2}} \\
& -4 \pi a N\left(1+\frac{\bar{x}}{a}\right) \frac{1}{S^{2}} \iint d S_{2} \iint d S_{1} \log R_{12} \\
& -4 \pi a N \frac{1}{S^{2}} \iint d S_{2} \iint d S_{1}\left[\left(\frac{1}{a}+\frac{2 u_{\mathrm{x}}}{u}\right) x_{1}+\frac{2 u_{\mathrm{y}}}{u} y_{1}\right] \log R_{12} \\
& +4 \pi a N\left(1+\frac{\bar{x}}{a}\right) \frac{1}{S^{2}} \iint d S_{2} \iint d S_{1} \sum_{n=1}^{\infty} b_{\mathrm{n}}\left(\frac{z}{2 \pi p}\right)^{2 \mathrm{n}} \\
& +4 \pi a N \frac{1}{S^{2}} \iint d S_{2} \iint d S_{1}\left[\left(\frac{1}{a}+\frac{2 u_{\mathrm{x}}}{u}\right) x_{1}+\frac{2 u_{\mathrm{y}}}{u} y_{1}\right] \sum_{n=1}^{\infty} b_{\mathrm{n}}\left(\frac{z}{2 \pi p}\right)^{2 \mathrm{n}} \\
& -4 \pi a \frac{1}{S^{2}} \iint d S_{2} \iint d S_{1} \sum_{n=2}^{\infty} c_{\mathrm{n}}\left(\frac{z}{2 \pi p}\right)^{2 \mathrm{n}}
\end{aligned}
$$

In this formula $\bar{x}$ is the positive distance from the surface of the cylindrical form to the center of gravity of the section, and $x_{1} y_{1}$ and $x_{2} y_{2}$ are now referred to this center of gravity as origin.

The principal term in this formula is

$$
L_{0} \equiv \frac{8 a}{\pi} A_{0} \frac{a^{2}}{p^{2}}=\frac{32 \pi N^{2} a^{3}}{3 l^{2}}\left\{\frac{\left(1-k^{2}\right) K(k)-\left(1-2 k^{2}\right) E(k)}{k^{3}}-1\right\}
$$

which is the well-known formula of Lorenz for the inductance of a continuous cylindrical current sheet of radius $a$ and length $l=2 \pi p N$ $\left(\right.$ where $\left.k^{2}=\frac{1}{1+\left(\frac{l}{2 a}\right)^{2}}\right)$ in which there is a circular component of cur-

rent only. (The axial length of each filament is $l=2 \pi p N$, that of the coil is $l+$ wire thickness). 
The inductance $\bar{L}_{0}$ of a current sheet (with circular component of current only) for a cylinder of the same length, but which passes through the center of gravity of the section of the wire would be given by the same formula in which $a$ is replaced by $a+\bar{x}$ and $k$ by $\bar{k}$ where

$$
\bar{k}=\frac{1}{\sqrt{1+\left[\frac{l}{2(a+\bar{x})}\right]^{2}}}=k+\frac{\bar{x}}{a} k\left(1-k^{2}\right)-\frac{3}{2} \frac{\bar{x}^{2}}{a^{2}} k^{3}\left(1-k^{2}\right)
$$

If we make use of the definitions (91 to (96) and expand to the second order, inclusive, we find

$$
\begin{gathered}
\bar{L}_{0} \equiv \frac{32 \pi N^{2}(a+\bar{x})^{3}}{3 l^{2}}\left\{\frac{\left(1-\bar{k}^{2}\right) K(\bar{k})-\left(1-2 \bar{k}^{2}\right) E(\bar{k})}{\bar{k}^{3}}-1\right\}= \\
=\frac{8 a}{\pi}\left[A_{0} \frac{a^{2}}{p^{2}}+2 A_{1} \frac{a \bar{x}}{p^{2}}+A_{3} \frac{\bar{x}^{2}}{p^{2}}\right]
\end{gathered}
$$

(In this formula $l$ is $2 \pi p N$ and not the overall length of the sheet.)

\section{WIRE OF SECTION $S$ SYMMETRICAL WITH RESPECT TO THE $x$ AND $y$ AXES THROUGH ITS CENTER OF GRAVITY}

Since $\log R_{12}$ and the real part of $z^{2 n}=\left[x_{2}-x_{1}+i\left(y_{2}-y_{1}\right)\right]^{2 n}$ are both symmetrical functions of the two points $P_{1}$ and $P_{2}$, it follows that the second and fourth integrals in (106) will vanish because of the assumed symmetry. Also $k_{x y}=0$. We then obtain from (106) the following formula for the inductance of a helix, in which the section of the wire is symmetrical about both axes through its center of gravity.

$$
\begin{aligned}
& L_{\mathrm{n}}=\bar{L}_{0}-4 \pi(a+\bar{x}) N\left[\log \frac{\overline{\bar{R}}}{p}-\sum_{n=1}^{\infty} \frac{(-1)^{\mathrm{n}}}{n} \overline{\left(\frac{z}{2 \pi p}\right)_{\text {real }}^{2 \mathrm{n}}} S_{2 \mathrm{n}}\right] \\
& +2 \pi a\left\{A_{2}+\frac{1}{3}\left[1+12 \frac{k_{\mathrm{x}^{2}}-k_{\mathrm{y}^{2}}}{(2 \pi p)^{2}}\right] \log \frac{a}{p}-2 \sum_{n=2}^{\infty} \frac{(-1)^{\mathrm{n}}}{n}{\overline{\left(\frac{z}{2 \pi p}\right)_{\text {real }}^{2 \mathrm{n}}}}_{S_{2 \mathrm{n}-1}(108)}\right. \\
& +8\left(\frac{E(k)}{k}-1\right)\left(1+\frac{2 a u_{x}}{u}\right) \frac{k_{x^{2}}}{(2 \pi p)^{2}} \\
& \left.+4\left[.18123+2\left(\frac{K(k)-E(k)}{k}\right)-k K(k)\right] \frac{k_{y^{2}}-k_{x^{\prime}}}{(2 \pi p)^{2}}\right\}
\end{aligned}
$$

where

$$
\begin{aligned}
& S_{\mathrm{n}} \equiv \sum_{k=1}^{\infty} \frac{1}{k^{\mathrm{n}}} \\
& S_{2}=1.644934 \\
& S_{6}=1.017343 \\
& S_{10}=1.000995 \\
& S_{3}=1.202057 \\
& S_{7}=1.008349 \\
& S_{11}=1.000494 \\
& S_{4}=1.082323 \\
& S_{8}=1.004077 \\
& S_{12}=1.000245 \\
& S_{5}=1.036928 \\
& S_{9}=1.002008
\end{aligned}
$$


In this formula $\overline{\bar{R}}$ denotes the geometric mean distance of the section from itself and is defined by

$$
\log \overline{\bar{R}}=\frac{1}{S^{2}} \iint \mathrm{d} S_{2} \iint d S_{1} \log R_{12}
$$

Similarly $\overline{\bar{Z}}_{\text {real }}{ }^{2 \mathrm{n}}$ denotes the mean value of the mean value of the real part of

where

$$
Z^{2 n}=R_{12}{ }^{2 n} \cos 2 n \phi_{12}
$$

It is defined by

$$
Z=x_{2}-x_{1}+c\left(y_{2}-y_{1}\right)=R_{12} c^{\mathrm{i} \phi_{12}}
$$

$\overline{\bar{Z}}_{\text {real }}{ }^{2 n}=\frac{1}{S^{2}} \iint d S_{2} \iint d S_{1} Z_{\text {real }}{ }^{2 n}=\frac{1}{S^{2}} \iint d S_{2} \iint d S_{1} R_{12}{ }^{2 n} \cos 2 n \phi_{12}$

For sections of any ordinary shape, this may be readily found by means of integrations similar to those needed to evaluate $k_{\mathrm{x}}$ and $k_{\mathrm{y}}$. - The terms involving $u_{\mathrm{y}}$ have canceled. The term $\frac{a u_{\mathrm{x}}}{u}$ is zero if the current density is uniform, but for the "natural distribution" the current density $u(x)=\frac{\text { constant }}{a+x}$ so that $\frac{a u_{x}}{u}=-1$. Hence the next to the last term in $(108)$ is $\pm 8\left(\frac{E(k)}{k}-1\right) \frac{k_{x^{2}}}{(2 \pi p)^{2}}$ according as the current distribution is constant or varies inversely as the length of the helical filament through the point. This term serves to indicate the error caused by neglect of the nonuniformity of current.

It may be noted that the formula (108) applies only to those wire sections in which no two points can be farther apart than $2 \pi p$, the pitch of the helix. A method of modifying the $b_{\mathrm{n}}$ and $c_{\mathrm{n}}$ series in those exceptional cases where this condition is not satisfied is indicated in Appendix 7.

We may now derive from (108) the two cases of greatest practical importance, namely, that in which the wire has a circular section and that of a rectangular shape.

(a) Wire of Circular Section.-Let the diameter of the wire be $\alpha$. Then

$$
\bar{x}=\frac{\alpha}{2} \text { and } k_{\mathrm{x}}=k_{\mathrm{y}}=\frac{\alpha^{2}}{16}
$$

Each term $\overline{\overline{Z_{\text {real }}^{2 n}}}$ is identically zero, for if

$$
z_{1}=x_{1}+c y_{1}=r_{1} e^{i \theta_{1}} \text { and } z_{2}=x_{2}+i y_{2}=r_{2} e^{i \theta_{2}}
$$

$z^{2 \mathrm{n}}=\left(z_{2}-z_{1}\right)^{2 \mathrm{n}}=r_{2}{ }^{2 \mathrm{n}} e^{\mathrm{i} 2 \mathrm{n} \theta_{2}} \Gamma(2 n+1) \sum_{k=0}^{\infty}(-1)^{\mathrm{k}}\left(\frac{r_{1}}{r_{2}}\right) \mathrm{k} \frac{e^{\mathrm{ik}\left(\theta_{1}-\theta_{2}\right)}}{\Gamma(k+1) \Gamma(2 n+1-k)}$ if

$r_{1}<r_{2}=r_{1}{ }^{2 n} e^{\mathrm{j} 2 \mathrm{n} \theta_{1}} \Gamma(2 n+1) \sum_{k=0}^{\infty}(-1)^{\mathbf{k}}\left(\frac{r_{2}}{r_{1}}\right)^{\mathbf{k}} \frac{e^{\mathrm{ik}\left(\theta_{2}-\theta_{1}\right)}}{\Gamma(k+1) \Gamma\left(2 n+1-\eta_{k}\right)}$ if $r_{1}>r_{2}$ 
This gives for a circular section

$$
\begin{aligned}
& \overline{\overline{Z^{2 n}}} \equiv \frac{1}{S} \iint d S_{2} \frac{1}{S} \iint d S_{1} z^{2 \mathrm{n}}=\frac{1}{S} \iint d S_{2} z_{2}{ }^{2 \mathrm{n}}=\frac{1}{S} \iint d S_{2} r_{2}{ }^{2 \mathrm{n}} e^{\mathrm{i} 2 \mathrm{n} \theta_{3}} \\
& =0 \text { if } n \neq 0
\end{aligned}
$$

Hence, the two series in (108) drop out.

It is readily found that for a circular section of diameter $\alpha$

$$
\log \overline{\bar{R}}=\log \frac{\alpha}{2}-\frac{1}{4}=\log \frac{\alpha}{2 \pi}+.89473
$$

Hence, we obtain from (108) the following formula for the inductance of a helix wound with curcular wire:

$$
\begin{aligned}
L_{\mathrm{h}}=\bar{L}_{0} & -2 \pi\left(a+\frac{\alpha}{2}\right)\left\{2 N\left(.89473-\log \frac{2 \pi p}{\alpha}\right)\right. \\
& \left.-\frac{1}{3} \log \frac{\alpha}{p}-A_{2}(k)-\frac{1}{2}\left(\frac{E(k)}{k}-1\right)\left(1+\frac{2 a u_{\mathrm{z}}}{u}\right)\left(\frac{\alpha}{2 \pi p}\right)^{2}\right\}
\end{aligned}
$$

When the diameter of the wire $\alpha$ is equal to the pitch of the helix $2 \pi p$, the wires touch. When $\frac{\alpha}{2 \pi p}=e^{-0,80473}=0.40872$, the principal correction term vanishes.

(b) Wire of Rectangular Section.-Let the length of the section in the $y$ direction (parallel to the axis of the solenoid) be $\beta$. The insulating space is $2 \pi p-\beta$. Let the thickness of the wire be $a$ (in $x$ direction). Then

$$
\bar{x}=\frac{\alpha}{2} \quad k_{x^{2}}=\frac{\alpha^{2}}{12} \quad k_{y^{\prime}}=\frac{\beta^{2}}{12}
$$

It is easily found that

$$
\begin{aligned}
\log \frac{\overline{\bar{R}}}{p}=\log \frac{\sqrt{\alpha^{2}+\beta^{2}}}{p} & +\frac{2}{3}\left(\frac{\alpha}{\beta} \tan ^{-1} \frac{\beta}{\alpha}+\frac{\beta}{\alpha} \tan ^{-1} \frac{\alpha}{\beta}\right)-\frac{25}{12} \\
& -\frac{1}{12}\left[\frac{a^{2}}{\beta^{2}} \log \left(1+\frac{\beta^{2}}{\alpha^{2}}\right)+\frac{\beta^{2}}{\alpha^{2}} \log \left(1+\frac{a^{2}}{\bar{\beta}^{2}}\right)\right]
\end{aligned}
$$

In terms of the diagonal of the rectangle, $\rho=\sqrt{a^{2}+\beta^{2}}$ and of the acute angle, $\theta=\tan ^{-1} \frac{\alpha}{\beta}$, which it makes with the axis of the solenoid, this becomes

$$
\begin{aligned}
\log \frac{\overline{\bar{R}}}{p} & =\log \frac{\rho}{2 \pi p}+\frac{1}{6}\left(\tan ^{2} \theta \log \sin \theta+\cot ^{2} \theta \log \cos \theta\right) \\
& +\frac{2}{3}\left[\left(\frac{\pi}{2}-\theta\right) \tan \theta+\theta \cot \theta\right]-0.24545
\end{aligned}
$$


It is also found by elementary integration that

$$
\begin{aligned}
\overline{\left(\frac{Z}{2 \pi p}\right)_{\text {real }}^{2 \mathrm{n}}} & =4(-1)^{\mathrm{n}}\left(\frac{\rho}{2 \pi p}\right)^{2 \mathrm{n}} \frac{\left[\cos ^{2 \mathrm{n}+4} \theta+(-1)^{\mathrm{n}} \sin ^{2 \mathrm{n}+4} \theta-\cos (2 n+4) \theta\right]}{(2 n+1)(2 n+2)(2 n+3)(2 n+4) \sin ^{2} \theta \cos ^{2} \theta} \\
& \left.=-4(-1)^{\mathrm{n}}\left(\frac{2 \pi p}{\alpha}\right)^{2}\left(\frac{2 \pi p}{\beta}\right)^{2} \frac{\left[\left(\frac{\beta+i \alpha}{2 \pi p}\right)^{2 \mathrm{n}+4}-\left(\frac{\beta}{2 \pi p}\right)^{2 \mathrm{n}+4}-\left(\frac{i \alpha}{2 \pi p}\right)^{2 \mathrm{n}+4}\right]}{(2 n+1)(2 n+2)(2 n+3)(2 n+4)}\right] \\
& =16\left(\frac{\rho}{2 \pi p}\right)^{2 \mathrm{n}} \frac{\left[\frac{1}{2}^{\mathrm{n}+1}+(-1)^{\frac{n}{2}}\right]}{(2 n+1)(2 n+2)(2 n+3)(2 n+4)} \text { if } n \text { is even and } \theta \\
& =\frac{\pi}{4},=0 \text { if } n \text { is odd and } \theta=\frac{\pi}{4}
\end{aligned}
$$

We thus obtain from (108), when the wire has a rectangular section, the general formula

$$
\begin{aligned}
L_{\mathrm{n}}= & \bar{L}_{0}+2 \pi\left(a+\frac{\alpha}{2}\right)\left\{A_{2}(k)+\frac{1}{3}\left[1+\frac{\alpha^{2}-\beta^{2}}{(2 \pi p)^{2}}\right] \log \frac{a}{p}\right. \\
-2 N & {\left[-\log \frac{2 \pi p}{\rho}+\frac{1}{6}\left(\tan ^{2} \theta \log \sin \theta+\cot ^{2} \theta \log \cos \theta\right)\right.} \\
& +\frac{2}{3}\left(\left(\frac{\pi}{2}-\theta\right) \tan \theta+\theta \cot \theta\right)-.24545 \\
& \left.-\frac{8}{\sin ^{2} \theta \cos ^{2} \theta} \sum_{\mathrm{n}=1}^{\infty} S_{2 \mathrm{n}}\left(\frac{\rho}{2 \pi p}\right)^{2 \mathrm{n}} \frac{\cos ^{2 \mathrm{n}+4} \theta+(-1)^{\mathrm{n}} \sin ^{2 \mathrm{n}+4} \theta-\cos (2 n+4) \theta}{2 n(2 n+1)(2 n+2)(2 n+3)(2 n+4)}\right] \\
& +\frac{2}{3}\left(\frac{E(k)}{k}-1\right)\left(1+\frac{2 a u_{\mathrm{x}}}{n}\right)\left(\frac{\alpha}{2 \pi p}\right)^{2}+\frac{1}{3}[\cdot 18123 \\
& \left.+2\left(\frac{K-E}{k}\right)-k K\right] \cdot \frac{\beta^{2}-\alpha^{2}}{(2 \pi p)^{2}} \\
- & \left.\frac{16}{\sin ^{2} \theta \cos ^{2} \theta} \sum_{n=2}^{\infty} S_{2 \mathrm{n}-1}\left(\frac{\rho}{2 \pi p}\right)^{2 \mathrm{n}} \frac{\cos ^{2 \mathrm{n}+4} \theta+(-1)^{\mathrm{n}} \sin ^{2 \mathrm{n}+4} \theta}{2 n(2 n+1)(2 n+2)(2 n+3)}\right\}
\end{aligned}
$$

provided $\rho \leq 2 \pi p$. If in exceptional cases $\rho>2 \pi p$, these series may be modified by taking out the cause of the divergence, which is the term $\log \left[1+\left(\frac{z}{2 \pi p}\right)^{2}\right]$. The remaining series will then converge if $|z|<4 \pi p$, as explained in Appendix 7. 
It is seen by (109) that $S_{\mathrm{n}}$ approaches 1 with increasing $n$. Hence to obtain a more rapidly converging series than the above, we may notice that if $S_{2 n}$ were replaced by 1 the two series in (108) would become summable, for by repeated integration from $x=0$ to $x=x$ of the series

$$
\sum_{n=1}^{\infty} \frac{x^{2 \mathrm{n}}}{2 n}=-\frac{1}{2} \log \left(1-x^{2}\right)
$$

one finds that

$$
\begin{aligned}
& 8 \sum_{n=1}^{\infty} \frac{x^{2 n+4}}{2 n(2 n+1)(2 n+2)(2 n+3)(2 n+4)}=\frac{7 x^{2}}{6}+\frac{25}{36} x^{4} \\
& -\frac{(1+x)^{4} \log (1+x)+(1-x)^{4} \log (1-x)}{6}
\end{aligned}
$$

and from this one finds that the real part of

$$
\begin{aligned}
& 8\left(\frac{2 \pi p}{\alpha}\right)^{2}\left(\frac{2 \pi p}{\beta}\right)^{2} \sum_{n=1}^{\infty} \frac{\left(\frac{\beta+i \alpha}{2 \pi p}\right)^{2 n+4}-\left(\frac{\beta}{2 \pi p}\right)^{2 n+4}-\left(\frac{i \alpha}{2 \pi p}\right)^{2 n+4}}{2 n+1)(2 n+2)(2 n+3)(2 n+4)}=-\frac{25}{6} \\
& -\frac{1}{6}\left(\frac{2 \pi p}{\alpha}\right)^{2}\left(\frac{2 \pi p}{\beta}\right)^{2}\left[f\left(\frac{\alpha}{2 \pi p}, \frac{\beta}{2 \pi p}\right)-f\left(\frac{\alpha}{2 \pi p}, o\right)-f\left(0, \frac{\beta}{2 \pi p}\right)\right]
\end{aligned}
$$

where

$$
\begin{aligned}
f\left(\frac{\alpha}{2 \pi p}, \frac{\beta}{2 \pi p}\right) & =\left[\left(1+\frac{\beta}{2 \pi p}\right)^{4}-6\left(1+\frac{\beta}{2 \pi p}\right)^{2}\left(\frac{\alpha}{2 \pi p}\right)^{2}\right. \\
& \left.+\left(\frac{\alpha}{2 \pi p}\right)^{4}\right] \log \sqrt{\left(1+\frac{\beta}{2 \pi p}\right)^{2}+\left(\frac{\alpha}{2 \pi p}\right)^{2}} \\
& +\left[\left(1-\frac{\beta}{2 \pi p}\right)^{4}-6\left(1-\frac{\beta}{2 \pi p}\right)^{2}\left(\frac{\alpha}{2 \pi p}\right)^{2}\right. \\
& \left.+\left(\frac{\alpha}{2 \pi p}\right)^{4}\right] \log \sqrt{\left(1-\frac{\beta}{2 \pi p}\right)^{2}+\left(\frac{\alpha}{2 \pi p}\right)^{2}} \\
& -4\left(1+\frac{\beta}{2 \pi p}\right)\left(\frac{\alpha}{2 \pi p}\right)\left[\left(1+\frac{\beta}{2 \pi p}\right)^{2}\right. \\
& \left.-\left(\frac{\alpha}{2 \pi p}\right)^{2}\right] \tan ^{-1} \frac{\alpha}{2 \pi p+\beta} \\
& -4\left(1-\frac{\beta}{2 \pi p}\right)\left(\frac{\alpha}{2 \pi p}\right)\left[\left(1-\frac{\beta}{2 \pi p}\right)^{2}\right. \\
& \left.-\left(\frac{\alpha}{2 \pi p}\right)^{2}\right] \tan ^{-1} \frac{\alpha}{2 \pi p-\beta}
\end{aligned}
$$


so that

$$
\begin{gathered}
f\left(\frac{\alpha}{2 \pi p}, o\right)=2\left[1-6\left(\frac{\alpha}{2 \pi p}\right)^{2}+\left(\frac{\alpha}{2 \pi p}\right)^{4}\right] \log \sqrt{1+\left(\frac{\alpha}{2 \pi p}\right)^{2}} \\
-8\left(\frac{\alpha}{2 \pi p}\right)\left[1-\left(\frac{\alpha}{2 \pi p}\right)^{2}\right] \tan ^{-1} \frac{\alpha}{2 \pi p} \\
f\left(o, \frac{\beta}{2 \pi p}\right)=\left[1+\frac{\beta}{2 \pi p}\right]^{\log } \log \left[1+\left(\frac{\beta}{2 \pi p}\right)\right] \\
+\left[1-\frac{\beta}{2 \pi p}\right]^{1} \log \left[1-\left(\frac{\beta}{2 \pi p}\right)\right]
\end{gathered}
$$

Hence, the formula (120) may be written

$$
\begin{aligned}
L_{\mathrm{h}} & =\bar{L}_{0}+2 \pi\left(a+\frac{\alpha}{2}\right)\left\{A_{2}(k)+\frac{1}{3}\left[1+\frac{\alpha^{2}-\beta^{2}}{(2 \pi p)^{2}}\right] \log \frac{a}{p}\right. \\
& -2 N\left[-\log \frac{2 \pi p}{\rho}-4.41212+\frac{1}{6}\left(\tan ^{2} \theta \log \sin \theta+\cot ^{2} \theta \log \cos \theta\right)\right. \\
& +\frac{2}{3}\left(\left(\frac{\pi}{2}-\theta\right) \tan \theta+\theta \cot \theta\right) \\
& -\frac{f\left(\frac{\alpha}{2 \pi p}, \frac{\beta}{2 \pi p}\right)-f\left(\frac{\alpha}{2 \pi p}, o\right)-f\left(0, \frac{\beta}{2 \pi p}\right)}{6\left(\frac{\alpha}{2 \pi p}\right)^{2}\left(\frac{\beta}{2 \pi p}\right)^{2}} \\
& \left.-\frac{8}{\sin ^{2} \theta \cos ^{2} \theta} \sum_{n=1}^{\infty} \frac{\left(\frac{\rho}{2 \pi p}\right)^{2 \mathrm{n}}\left(\cos ^{2 \mathrm{n}+4} \theta+(-1)^{\mathrm{n}} \sin ^{2 \mathrm{n}+4} \theta\right.}{(2 n+1)(2 n+2)(2 n+3)(2 n+4)}\left(S_{2 \mathrm{n}}-1\right)\right] \\
& +\frac{2}{3}\left(\frac{E(k)}{k}-1\right)\left(1+\frac{2 a u_{\mathrm{x}}}{u}\right)\left(\frac{\alpha}{2 \pi p}\right)^{2} \\
& +\frac{1}{3}\left[1.18123+2\left(\frac{K-E}{k}\right)-k K\right]^{\frac{\beta^{2}-\alpha^{2}}{(2 \pi p)^{2}}} \\
& -\frac{25}{3}-\frac{f\left(\frac{\alpha}{2 \pi p}, \frac{\beta}{2 \pi p}\right)-f\left(\frac{\alpha}{2 \pi p}, o\right)-f\left(0, \frac{\beta}{2 \pi p}\right)}{3\left(\frac{\alpha}{2 \pi p}\right)^{2}\left(\frac{\beta}{2 \pi p}\right)^{2}} \\
& \left.-\frac{16}{\sin ^{2} \theta \cos ^{2} \theta} \sum_{n=2}^{\infty} \frac{\left(\frac{\rho}{2 \pi p}\right)^{2 n}\left(\cos ^{2 n+4} \theta+(-1)^{\mathrm{n}} \sin ^{2 \mathrm{n}+4} \theta\right.}{2 n+1)(2 n+2)(2 n+3)(2 n+4)}\left(S_{2 \mathrm{n}-1}-1\right)\right\}
\end{aligned}
$$

This formula is preferable to (120) because in it the two series converge more rapidly, since $S_{2 \mathrm{n}}-1$ and $S_{2 \mathrm{n}-1}-1$ approach zero with increasing $n$. 
In fact, it will be shown presently that in most cases the first two or three terms of the first series will be sufficient for a precision greater than 1 part in 1,000,000, while the second series may be replaced by its first term, or neglected entirely.

A special case of (126) is that in which the wire section is a square. Placing $\beta=\alpha$ and $\theta=\frac{\pi}{4}$ in (126) gives

$$
\begin{aligned}
L_{\mathrm{n}} & =\bar{L}_{0}+2 \pi\left(a+\frac{\alpha}{2}\right)\left\{A_{2}(k)+\frac{1}{3} \log \frac{a}{p}\right. \\
& +\frac{2}{3}\left(\frac{E(k)}{k}-1\right)\left(1+\frac{2 a u_{\mathrm{x}}}{u}\right)\left(\frac{\alpha}{2 \pi p}\right)^{2} \\
& -2 N\left[-3.48045-\log \frac{2 \pi p}{\rho}\right. \\
& -\frac{f\left(\frac{\alpha}{2 \pi p}, \frac{\alpha}{2 \pi p}\right)-f\left(\frac{\alpha}{2 \pi p}, 0\right)-f\left(0, \frac{\alpha}{2 \pi p}\right)}{6\left(\frac{\alpha}{2 \pi p}\right)^{4}} \\
& \left.-32 \sum_{n=1}^{\infty} \frac{\left(\frac{\rho}{2 \pi p}\right)^{4 \mathrm{n}}\left((-1)^{\mathrm{n}}+\frac{1}{2^{2 \mathrm{n}+1}}\right)\left(S_{4 \mathrm{n}}-1\right)}{4 n(4 n+1)(4 n+2)(4 n+3)(4 n+4)}\right] \\
& -\frac{25}{3}-\frac{f\left(\frac{\alpha}{2 \pi p}, \frac{\alpha}{2 \pi p}\right)-f\left(\frac{\alpha}{2 \pi p}, 0\right)-f\left(0, \frac{\alpha}{2 \pi p}\right)}{3\left(\frac{\alpha}{2 \pi p}\right)^{4}} \\
& \left.-64 \sum_{n=1}^{\infty} \frac{\left(\frac{\rho}{2 \pi p}\right)^{4 \mathrm{n}}\left((-1)^{\mathrm{n}}+\frac{1}{2^{2 \mathrm{n}+1}}\right)\left(S_{4 \mathrm{n}-1}-1\right)}{4 n(4 n+1)(4 n+2)(4 n+3)(4 n+4)}\right\}
\end{aligned}
$$

Another special case of (126) is that in which the wire is a thin tape. Placing $\alpha=0, \rho=\beta$, and $\theta=0$ in (126) gives 


$$
\begin{aligned}
L_{\mathrm{n}} & =L_{0}+2 \pi a\left\{A_{2}(k)+\frac{1}{3}\left[1-\left(\frac{\beta}{2 \pi p}\right)^{2}\right] \log \frac{a}{p}\right. \\
& +\frac{1}{3}\left[1.18123+2\left(\frac{K-E}{k}\right)-k K\right]\left(\frac{\beta}{2 \pi p}\right)^{2}+2 N\left[2.66212+\log \frac{2 \pi p}{\beta}\right. \\
& -\frac{\left(1+\frac{\beta}{2 \pi p}\right)^{2} \log \left(1+\frac{\beta}{2 \pi p}\right)+\left(1-\frac{\beta}{2 \pi p}\right)^{2} \log \left(1-\frac{\beta}{2 \pi p}\right)}{\left(\frac{\beta}{2 \pi p}\right)^{2}} \\
& \left.+4 \sum_{n=1}^{\infty} \frac{\left.\left(\frac{\beta}{2 \pi p}\right)^{2 n}\left(S_{2 n}-1\right)\right]}{2 n(2 n+1)(2 n+2)}\right] \\
& -6+2 \frac{\left(1+\frac{\beta}{2 \pi p}\right)^{2} \log \left(1+\frac{\beta}{2 \pi p}\right)^{2}\left(1-\frac{\beta}{2 \pi p}\right)^{2} \log \left(1-\frac{\beta}{2 \pi p}\right)}{\left(\frac{\beta}{2 \pi p}\right)^{2}} \\
& \left.-8 \sum_{n=2}^{\infty} \frac{\left(\frac{\beta}{2 \pi p}\right)^{2 n}\left(S_{2 n-1}-1\right)}{2 n(2 n+1)(2 n+2)}\right\}
\end{aligned}
$$

If in this equation we let $\beta=2 \pi p$, this gives the inductance of a continuous current sheet. The bracket multiplying $2 N$ is found to ranish, so that for a continuous current sheet

$$
\begin{aligned}
L_{\mathrm{hB}} & =L_{0}+2 \pi a\left\{A_{2}(k)+\frac{1}{3}\left[2\left(\frac{K-E}{k}\right)-k K\right]-.0610\right. \\
& -8 \sum_{n=2}^{\infty} \frac{S_{2 \mathrm{n}-1}-1}{2 n(2 n+1)(2 n+2)} \\
& \left.+2 N\left[-.11048+4 \sum_{n=1}^{\infty} \frac{S_{2 \mathrm{n}}-1}{2 n(2 n+1)(2 n+2)}\right]\right\}
\end{aligned}
$$

Or

$$
L_{\mathrm{hs}}=L_{0}+2 \pi a\left\{\mathrm{~A}_{2}(k)-.0755+\frac{1}{3}\left[2\left(\frac{K-E}{k}\right)-k K\right]\right\}
$$

This quantity $L_{\mathrm{ng}}$ is the inductance of a continuous helical current sheet consisting of tape windings without insulation space. The term $L_{0}$ is that of a truly cylindrical current sheet winding in which 
there is a circular component of current only. The largest part of the remaining terms is the term

$$
\frac{8 a B_{0}}{\pi}=\frac{8 a}{\pi} \int_{0}^{\frac{\sqrt{1-k^{2}}}{k}} d \eta_{2} \int_{0}^{\eta_{2}} d \eta_{1} \frac{K\left(\frac{1}{\sqrt{1+\eta_{1}^{2}}}\right)}{\sqrt{1+\eta_{1}^{2}}}
$$

which is the principal term of $2 \pi a A_{2}(k)$ (equations 89 and 90). This term is the contribution of the axial or $y$ component of current in the cylinder, as shown in Appendix 9. In Appendix 10 it is shown that the negative of this, $-\frac{8 a B_{0}}{\pi}$, represents the mutual inductance between the helix and a return wire very close to the helix. The remaining terms in (129) are relatively small and represent the end effects due to the fact that the helical sheet differs slightly in shape from the true cylinder.

A confirmation of this formula (129) for a helical current sheet may be obtained by making use of the fact (Appendix 7) that $F_{2}\left(\frac{z}{p}\right)-\frac{1}{2}\left(\frac{z}{p}\right)=-\log 2 \sinh \frac{z}{2 p}$. When $x_{1}=x_{2}=0$ this becomes $-\log 2 \sin \frac{\left|y_{2}-y_{1}\right|}{2 p}$ and the formula (108) then gives for the inductance of a helical tape winding the expression

$$
\begin{aligned}
L_{\mathrm{h}} & =L_{0}+2 \pi a\left\{A_{2}(k)+\frac{1}{3}\left[.18123-2\left(\frac{K-E}{k}\right)-k K\right]\left(\frac{\beta}{2 \pi p}\right)^{2}\right. \\
+ & \frac{1}{3}\left[1-\left(\frac{\beta}{2 \pi p}\right)^{2}\right] \log \frac{a}{p}-8 \sum_{n=2}^{\infty}\left(\frac{\beta}{2 \pi p}\right)^{2 \mathrm{n}} \frac{S_{2 \mathrm{n}-1}}{2 n(2 n+1)(2 n+2)} \\
& \left.-2 N\left[\log 2+\frac{8 p^{2}}{\beta^{2}} \int_{0}^{\beta / 2 \mathrm{p}} d \theta\left(\frac{\beta}{2 p}-\theta\right) \log \sin \theta\right]\right\}
\end{aligned}
$$

If we add to this the identically zero quantity

$$
\begin{aligned}
\frac{1}{3}\left(\frac{\beta}{2 \pi p}\right)^{2} & -6+2 \frac{\left(1+\frac{\beta}{2 \pi p}\right)^{2} \log \left(1+\frac{\beta}{2 \pi p}\right)+\left(1-\frac{\beta}{2 \pi p}\right)^{2} \log \left(1-\frac{\beta}{2 \pi p}\right)}{\left(\frac{\beta}{2 \pi p}\right)^{2}} \\
& +8 \sum_{n=2}^{\infty} \frac{\left(\frac{\beta}{2 \pi p}\right)^{2 n}}{2 n(2 n+1)(2 n+2)}=0
\end{aligned}
$$




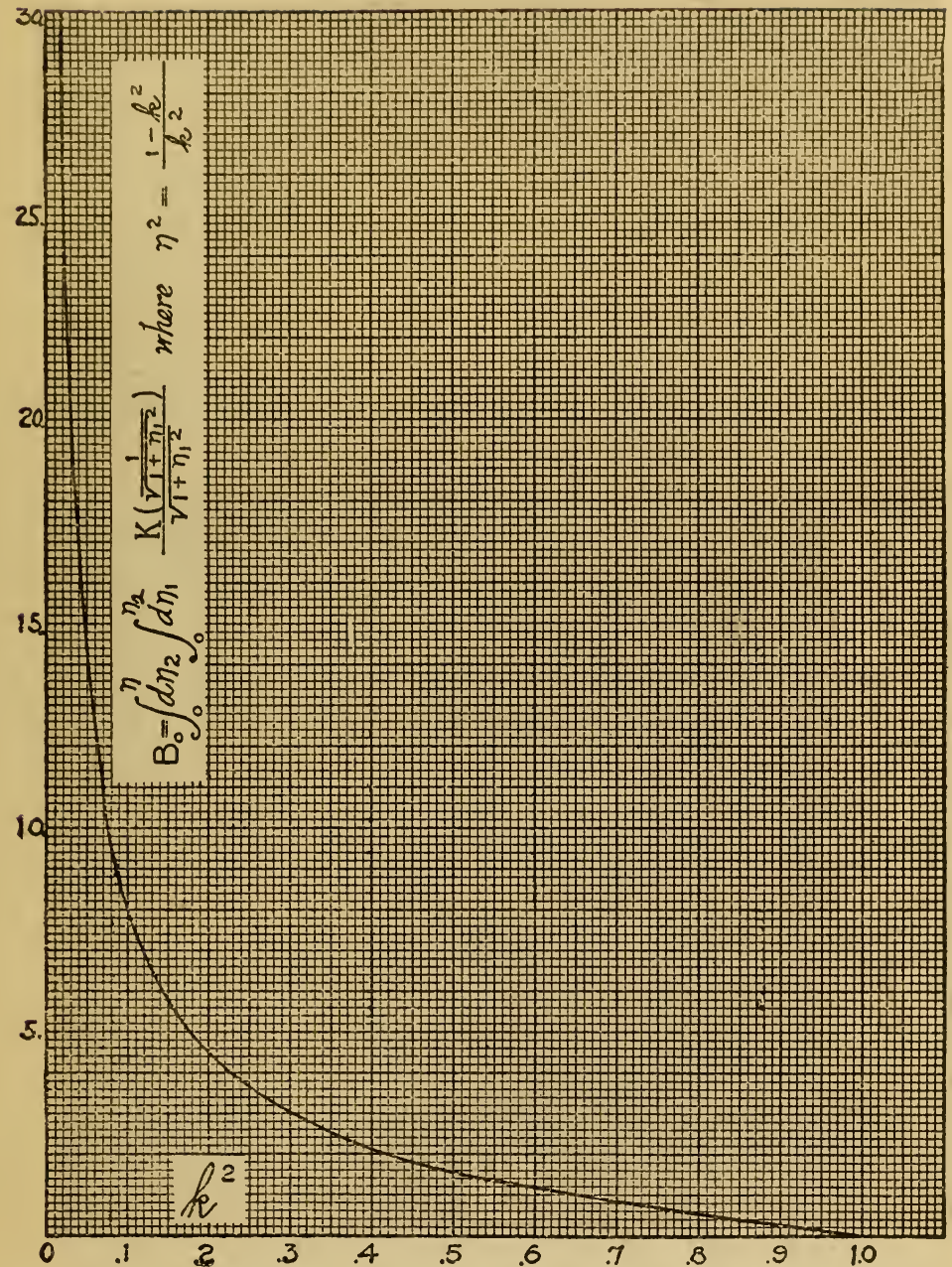

FIG. 2.-The function $B_{0}$ of equation (90) 


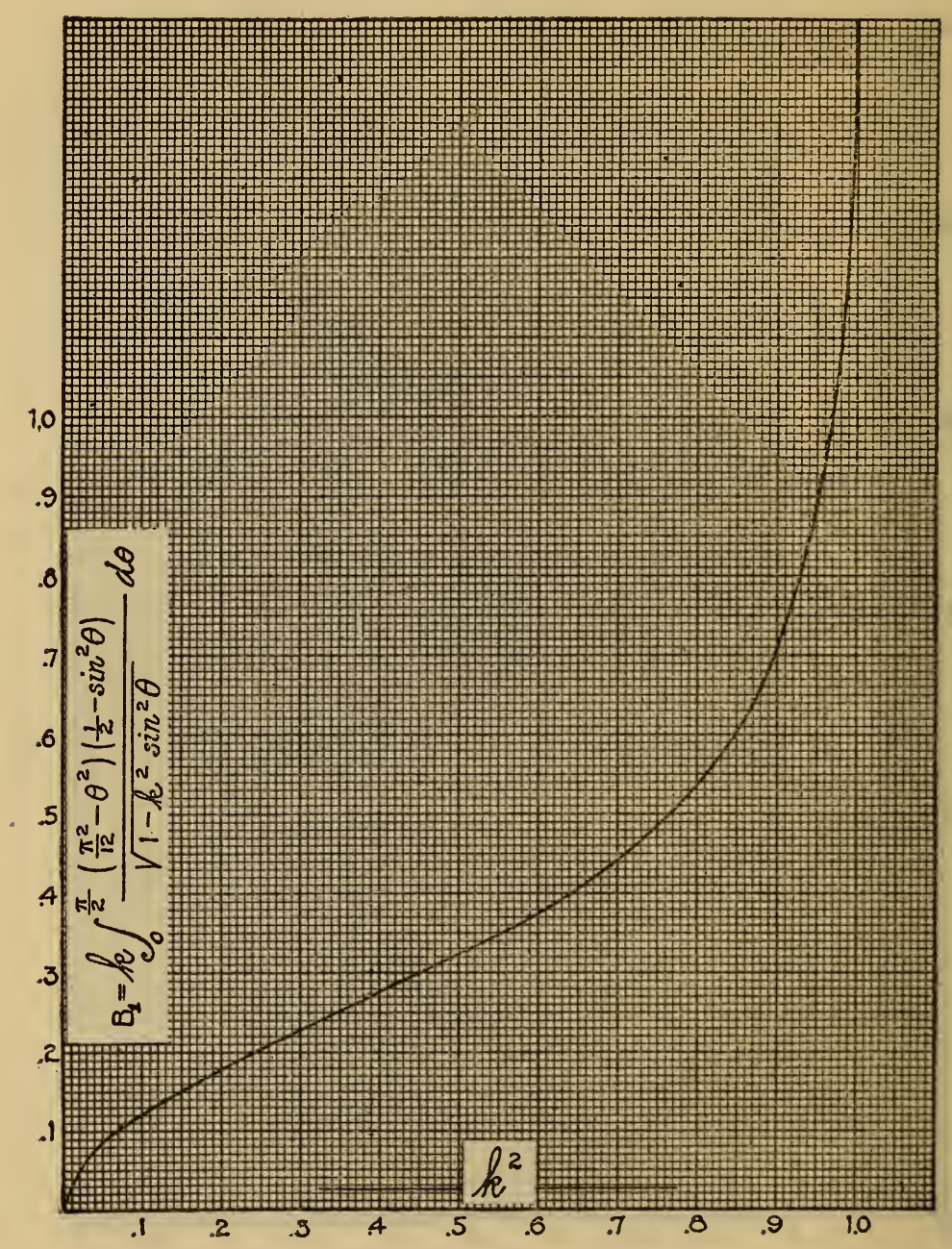

FIG. 3.-The function $B_{1}$ of equation (90) 
it becomes

$$
\begin{aligned}
L_{\mathrm{n}} & =L_{0}+2 \pi a\left\{\mathrm{~A}_{2}(k)+\frac{1}{3}\left[1-\left(\frac{\beta}{2 \pi p}\right)^{2}\right] \log \frac{a}{p}\right. \\
& +\frac{1}{3}\left[1.18123+2\left(\frac{K-E}{k}\right)-k K\right]\left(\frac{\beta}{2 \pi p}\right)^{2} \\
& -2 N\left[\log 2+\frac{8 p^{2}}{\beta^{2}} \int^{\beta / 2 \mathrm{p}} d \theta\left(\frac{\beta}{2 p}-\theta\right) \log \sin \theta\right]-6 \\
& +2 \frac{\left(1+\frac{\beta}{2 \pi p}\right)^{2} \log \left(1+\frac{\beta}{2 \pi p}\right)+\left(1-\frac{\beta}{2 \pi p}\right)^{2} \log \left(1-\frac{\beta}{2 \pi p}\right)}{\left(\frac{\beta}{2 \pi p}\right)^{2}} \\
& \left.-8 \sum_{n=2}^{\infty}\left(\frac{\beta}{2 \pi p}\right)^{2 \mathrm{n}} \frac{\left(S_{2 \mathrm{n}-1}-1\right)}{2 n(2 n+1)(2 n+2)}\right\}
\end{aligned}
$$

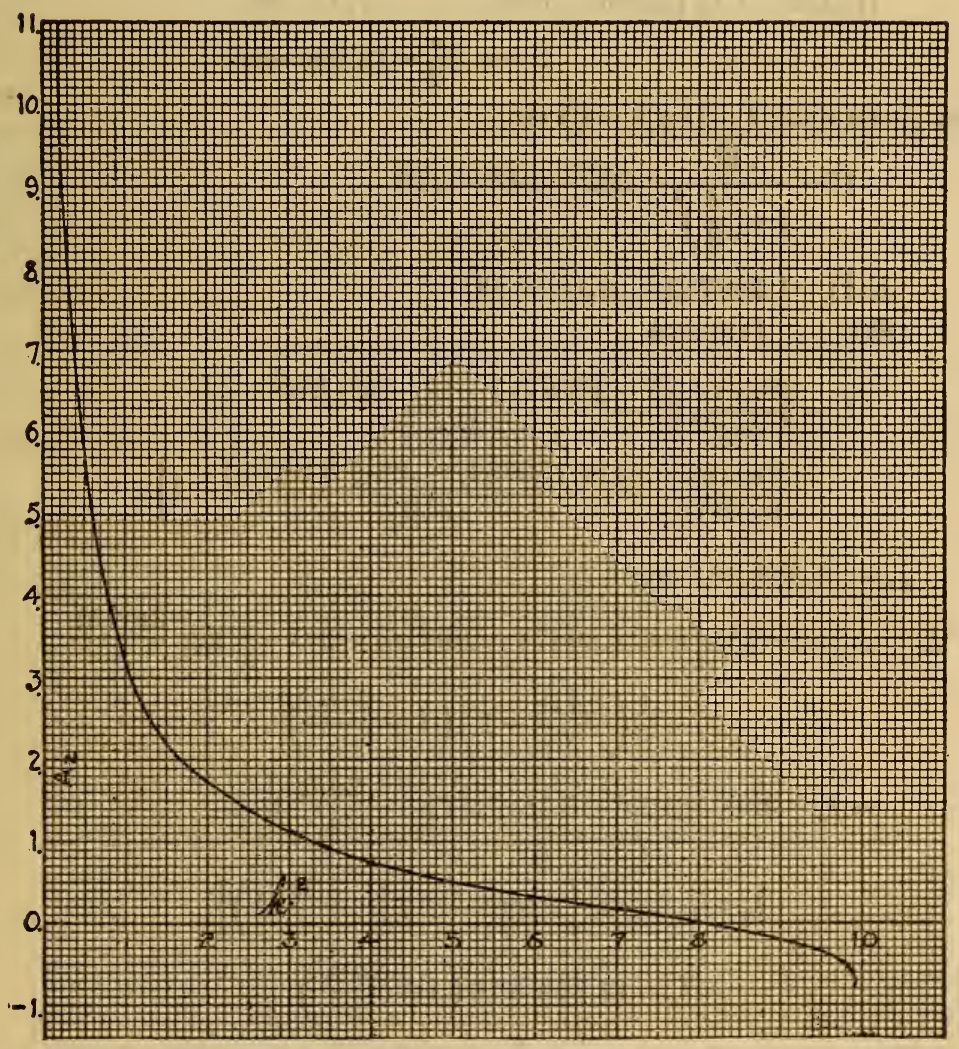

FIG. 4.-The function $A_{2}$ of equation (89) 
Equation (130) is identical with (128). If we place $\beta=2 \pi p$ in (130) it reduces to (129), since the integral

$$
\frac{2}{\pi^{2}} \int_{0}^{\pi}(\pi-\theta) \log \sin \theta d \theta=-\log 2
$$

and the terms multiplying $2 N$ vanish identically.

The function $A_{2}(k)$ is plotted against $k$ in Figure 4. It is obtained by (90) in terms of $B_{0}$ and $B_{1}$, which were obtained by graphical integration and are shown in Figures 2 and 3, respectively. A more accurate evaluation of $B_{0}$ could be made by means of the series given in Appendix 9

\section{NUMERICAL EXAMPLES}

(a) Circular Wire (Formula 114)-

$\left.\begin{array}{l}\text { Diameter of wire }=a=\frac{1}{2} \mathrm{~mm}=.05 \mathrm{~cm} \\ \text { Pitch of helix }=2 \pi p=1 \mathrm{~mm}=.10 \mathrm{~cm}\end{array}\right\} \frac{2 \pi p}{a}=2$

Number of turns $=N=400$

Radius of cylindrical form $=a=14.975 \mathrm{~cm}$

$$
\bar{a}=a+\frac{a}{2}=15.000 \mathrm{~cm}
$$

From these data $l=2 \pi p N=40 \mathrm{~cm}$

or

$$
\begin{aligned}
\bar{k}^{2} & =\frac{1}{1+\left(\frac{4}{3}\right)^{2}}=.36 \bar{k}=.6 \overline{k^{3}}=.216 \\
K(\bar{k}) & =1.7507538, A_{2}(\bar{k})=.90 \\
E(\bar{k}) & =1.4180834 \\
\bar{L}_{0} & =\frac{32 \pi(\bar{a})^{3}}{3(2 \pi p)^{2}}\left\{\frac{\left(1-\bar{k}^{2}\right) K(\bar{k})-\left(1-2 \overline{k^{2}}\right) E(\bar{k})}{\bar{k}^{3}}-1\right\} \\
& =\frac{32 \pi(15)^{3}}{3(.01)}\left\{\frac{.64(1.750754)-.28(1.418083)}{.216}-1\right\}
\end{aligned}
$$

$$
\begin{aligned}
\bar{L}_{0}=26,568,401 \mathrm{~cm} & \\
L_{\mathrm{h}}=\bar{L}_{0}-2 \pi(15)\{ & 800(.89473-.69315)-.90-\frac{1}{3} \log \frac{2 \pi(15)}{.1} \\
& \left.\quad-\frac{1}{8}\left(\frac{1.418}{.6}-1\right)\left(1+\frac{2 a u_{\mathrm{x}}}{u}\right)\right\} \\
= & \bar{L}_{0}-30 \pi\left\{161.26-3.18-.17\left(1+\frac{2 a u_{\mathrm{x}}}{u}\right)\right\}
\end{aligned}
$$

or

or

$$
\begin{aligned}
L_{\mathrm{h}} & =\bar{L}_{0}-30 \pi\left\{158.08-.17\left(1+\frac{2 a u_{\mathrm{x}}}{u}\right)\right\} \\
& =\bar{L}_{0}-14,899+16\left(1+\frac{2 a u_{\mathrm{x}}}{u}\right)
\end{aligned}
$$

$$
L_{\mathrm{h}}=26,553,502+16\left(1+\frac{2 a u_{\mathrm{x}}}{u}\right) \mathrm{cm}
$$


If the current distribution is uniform $\frac{u_{x}}{u}=0$ and this adds $16 \mathrm{~cm}$ to the above. If the current density varies inversely as the length of the helical filament $\frac{2 a u_{\mathrm{x}}}{u}=-2$ and this subtracts $16 \mathrm{~cm}$ from the abore. The two distributions differ in inductance by $32 \mathrm{~cm}$ or about 1 part in 1,000,000. Rosa's formula gives

$$
L_{\mathrm{h}}=\bar{L}_{0}-4 \pi a N\left(.55687-\log \frac{2 \pi p}{a}+B_{400}\right)
$$

or

$$
\begin{aligned}
L_{\mathrm{h}} & =\bar{L}_{0}-24,000(.55687-.69315+.3351) \\
& =\bar{L}_{0}-14,991=26,553,410 \mathrm{~cm}
\end{aligned}
$$

In this particular example, Rosa's formula is therefore lower than that here obtained for the case of uniform current distribution by 4 parts in $1,000,000$.

(b) Square Wire.-To illustrate the formula (127) for square wire, and to obtain a comparison with the preceding example, we shall assume that the thickness $\alpha$ of the wire is the same as the diameter of the round wire, the pitch, $2 \pi p$, number of turns $N$, and radius of form $a$ being also the same as before. Hence $\bar{k}$ and $\bar{L}_{0}, A_{2}$, and $\log \frac{a}{p}$ have the same values as before. Then for this square wire (127 becomes

$$
\begin{aligned}
& L_{\mathbf{h}}=\bar{L}_{0}-24000 \pi\left\{-3.82702-\frac{8}{3}\left(f\left(\frac{1}{2}, \frac{1}{2}\right)-f\left(\frac{1}{2}, 0\right)-f\left(0, \frac{1}{2}\right)\right)-32 P\right\} \\
& +30 \pi\left\{-5.15-\frac{16}{3}\left(f\left(\frac{1}{2}, \frac{1}{2}\right)-f\left(\frac{1}{2}, 0\right)-f\left(0, \frac{1}{2}\right)\right)-64 Q\right\}+21\left(1+\frac{2 a u_{\mathrm{x}}}{u}\right)
\end{aligned}
$$

where

$$
\begin{aligned}
& P=\sum_{n=1}^{\infty}\left(\frac{1}{2}\right)^{2 \mathrm{n}} \frac{\left((-1)^{\mathrm{n}}+\frac{1}{2^{2 \mathrm{n}+1}}\right)\left(\mathrm{S}_{4 \mathrm{n}}-1\right)}{4 n(4 n+1)(4 n+2)(4 n+3)(4 n+4)} \\
& Q=\sum_{n=1}^{\infty}\left(\frac{1}{2}\right)^{2 \mathrm{n}} \frac{\left((-1)^{\mathrm{n}}+\frac{1}{2^{2 \mathrm{n}+1}}\right)\left(S_{4 \mathrm{n}-1}-1\right)}{4 n(4 n+1)(4 n+2)(4 n+3)(4 n+4)} \\
& L_{\mathrm{n}}=\bar{L}_{0}-24000 \pi\left\{-3.82702+\frac{8}{3}(1.56268)-32 P\right\} \\
&+30 \pi\{3.18-64 Q\}+21\left(1+\frac{2 a u_{\mathrm{x}}}{u}\right) \\
&=\bar{L}_{0}-25345+21\left(1+\frac{2 a u_{\mathrm{x}}}{u}\right) \mathrm{cm} \\
&=26,543,070 \text { if } u_{\mathrm{x}}=0 \\
& 102333^{\circ}-26 \dagger-4
\end{aligned}
$$


The inductance of the helix with square wire is less than the corresponding one with circular wire of the same thickness by 1 part in 2,600. Here again Rosa's value is too low in this case by 6 parts in 1,000,000. I am indebted to Dr. F. W. Grover, of Union College, for the computation in this case by Rosa's method. The errors in Rosa's formula would become more important with increase in pitch of the helix or curvature of the solenoid. The formulas here obtained contain definite correction terms to the second order in both of these small quantities, and therefore permit of the use of smaller solenoids for standards while maintaining the necessary precision.

It has been shown that even in the most unfavorable case (that of a continuous helical current sheet) the second series in (128) may be entirely neglected, as it amounts to about 1 part in $25,000,000$. The first series may be replaced by the two terms corresponding to $n=1$ and $n=2$ without in any case making an error as large as 1 part in $1,000,000$. We may therefore write the formula (126) for the inductance of a helix wound with rectangular wire in a simpler form.

\section{SIMPLIFIED FORMULA FOR INDUCTANCE OF A HELIX WITH} RECTANGULAR WIRE

$$
\begin{aligned}
L_{\mathrm{h}} & =\bar{L}_{0}-4 \pi\left(a+\frac{\alpha}{2}\right) N\left\{\log \sqrt{x^{2}+y^{2}}-4.4121+\frac{2}{3}\left(\frac{x}{y} \tan ^{-1} \frac{y}{x}+\frac{y}{x} \tan ^{-1} \frac{x}{y}\right)\right. \\
& +.1075\left(x^{2}-y^{2}\right)-.00137\left[2\left(x^{4}+y^{4}\right)-5 x^{2} y^{2}\right] \\
& \left.+\frac{1}{6 x^{2} y^{2}}[F(x, o)+F(o y)-F(x, y)]\right\} \\
& +\frac{2 \pi a}{3}\left\{-25+3 A_{2}(k)+2 x^{2}\left(\frac{E(k)}{k}-1\right)\left(1+\frac{2 a u_{x}}{u}\right)\right. \\
& -\left[1.18+2\left(\frac{K-E}{k}\right)-k K\right]\left(x^{2}-y^{2}\right)+\left[1+x^{2}-y^{2}\right] \log \frac{a}{p} \\
& \left.+\frac{f(x, o)+f(o, y)-f(x, y)}{x^{2} y^{2}}\right\}
\end{aligned}
$$

where

$$
\begin{gathered}
x=\frac{\alpha}{2 \pi p}, \quad y=\frac{\beta}{2 \pi p} \text { and } x^{2}+y^{2} \leqq 1 \\
F(x, y)=f(x, y)+\left(x^{4}+y^{4}\right) \log \sqrt{x^{2}+y^{2}} \\
f(x, y)=\left[(1+y)^{4}-6 x^{2}(1+y)^{2}+x^{4}\right] \log \sqrt{(1+y)^{2}+x^{2}} \\
+\left[(1-y)^{4}-6 x^{2}(1-y)^{2}+x^{4}\right] \log \sqrt{(1-y)^{2}+x^{2}} \\
-4 x(1+y)\left[\left(1+y^{2}\right)-x^{2}\right] \tan ^{-1} \frac{x}{1+y} \\
-4 x(1-y)\left[(1-y)^{2}-x^{2}\right] \tan ^{-1} \frac{x}{1-y}
\end{gathered}
$$


The function $A_{2}(k)$ is plotted against $k^{2}=\frac{1}{1+\left(\frac{l}{2 a}\right)^{2}}$ in Figure 4. If the bracket which multiplies $4 \pi\left(a+\frac{\alpha}{2}\right) N$ in this equation be computed with an error of 0.0001 and that multiplying $\frac{2 \pi a}{3}$ with an error of 0.01 , the error in $L_{\mathrm{h}}$ will in general be less than 1 part in 1,000,000. A computation of the inductance of the helix wound with square wire has also been made by formula (131) with the same data as in example 2, and the result differs from that of the preceding computation by 1 part in $2,000,000$.

\section{APPENDIXES}

\section{Appendix 1.-EXPANSION OF CERTAIN HYPERGEOMETRIC FUNCTIONS}

The differential equation,

$$
x(1-x) \frac{d^{2} y}{d x^{2}}+[\gamma-(\alpha+\beta+1) x] \frac{d y}{d x}-\alpha \beta y=0
$$

where $\gamma-1$ is positive but not integral, has the general solution ${ }^{3}$ in the range $|x|<1$.

$$
y=C_{1} F(\alpha, \beta, \gamma, x)+C_{2} x^{1-\gamma} F(\alpha+1-\gamma, \beta+1-\gamma, 2-\gamma, x)
$$

provided that neither $\gamma$ nor $2-\gamma$ is equal to zero or a negative integer. In the range $|1-x|<1$ there is a particular solution

$$
y_{1} \equiv F(\alpha, \beta, \alpha+\beta-\gamma+1,1-x)
$$

which has a meaning if $\alpha+\beta-\gamma$ is not equal to a negative integer. Hence, in the common part of these two ranges, the function $y_{1}$ must be of the form (2); that is,

$$
\begin{aligned}
y_{1} \equiv & F(\alpha, \beta, \alpha+\beta-\gamma+1,1-x)=C_{1} F(\alpha, \beta, \gamma, x) \\
& +C_{2} x^{1-\gamma} F(\alpha+1-\gamma, \beta+1-\gamma, 2-\gamma, x)
\end{aligned}
$$

where $C_{1}$ and $C_{2}$ are numerical constants to be found. It is known that

and that

$$
F(\alpha, \beta, \gamma, 1)=\frac{\Gamma(\gamma) \Gamma(\gamma-\alpha-\beta)}{\Gamma(\gamma-\alpha) \Gamma(\gamma-\beta)} \text { when } \gamma-\alpha-\beta>0
$$

$$
(1-x)^{\alpha+\beta-\lambda} F(\alpha, \beta, \gamma, x)=F(\gamma-\alpha, \gamma-\beta, \gamma, x)
$$

Reimann-Weber, Partial Diff. Gleichung, 2, pp. 4-39. 
If $\alpha+\beta-\gamma>0$ and we let $x$ approach 1 in (6), then the second member will approach the finite value $\frac{\Gamma(\gamma) \Gamma(\alpha+\beta-\gamma)}{\Gamma(\alpha) \Gamma(\beta)}$ and this shows that the function $F(\alpha, \beta, \gamma, x)$ becomes infinite when $x$ approaches 1 , and in such a manner that

$$
\begin{aligned}
& \operatorname{limit}_{x=1}(1-x)^{\alpha+\beta-\gamma F}(\alpha, \beta, \gamma, x)=\frac{\Gamma(\gamma) \Gamma(\alpha+\beta-\gamma)}{\Gamma(\alpha) \Gamma(\beta)} \\
& \text { if } \quad \alpha+\beta-\gamma>0
\end{aligned}
$$

Similarly, it is found that

$$
\operatorname{limit}_{x=1} \frac{F(\alpha, \beta, \gamma, x)}{\log (1-x)}=-\frac{\Gamma(\gamma)}{\Gamma(\alpha) \Gamma(\beta)} \text { if } \alpha+\beta-\gamma=0
$$

By the use of (7) when $\alpha+\beta-\gamma>0$ or ( $\left.7^{\prime}\right)$ when $\alpha+\beta-\gamma=0$ we may determine the constants $C_{1}$ and $C_{2}$ in (4) as follows: Multiply (4) by

$$
\frac{(1-x)^{\alpha+\beta-\gamma}}{\Gamma(\alpha+\beta-\gamma)} \text { if } \alpha+\beta-\gamma>0
$$

or by $\frac{1}{\log (1-x)}$ if $\alpha+\beta-\gamma=0$. If we then let $x$ approach 1 , this gives in either case

$$
O=C_{1} \frac{\Gamma(\gamma)}{\Gamma(\alpha) \Gamma(\beta)}+C_{2} \frac{\Gamma(2-\gamma)}{\Gamma(\alpha+1-\gamma) \Gamma(\beta+1-\gamma)}
$$

Next multiply (4) by $x^{\gamma-1}$ and let $x=0$. This gives in either case

$$
C_{2}=\frac{\Gamma(\alpha+\beta-\gamma+1) \Gamma(\gamma-1)}{\Gamma(\alpha) \Gamma(\beta)}
$$

Hence, when $\gamma-1$ is positive, but not integral, while $\alpha+\beta-\gamma \geqq 0$, one finds from (8) and (9)

$$
C_{1}=-\frac{\Gamma(2-\gamma) \Gamma(\alpha+\beta-\gamma+1) \Gamma(\gamma-1)}{\Gamma(\gamma) \Gamma(\alpha+1-\gamma) \Gamma(\beta+1-\gamma)}
$$

If we let $\gamma-1=\nu$ and make use of the formula

$$
\Gamma(\nu)=\frac{\pi}{\sin \pi \nu \Gamma(1-\nu)}=\frac{(-1)^{\mathrm{n}} \pi}{\sin \pi(\nu-n) \Gamma(1-\nu)}
$$

where $n$ may be zero or any positive integer, then the identity (4) becomes with these values of $C_{1}$ and $C_{2}$

$$
\begin{aligned}
& y_{1}(x) \equiv \frac{\Gamma(\alpha-\nu) \Gamma(\beta-\nu) \Gamma(\alpha) \Gamma(\beta) F(\alpha, \beta, \alpha+\beta-\nu, 1-x)}{\Gamma(\alpha+\beta-\nu)}=\frac{(-1)^{\mathrm{n}} \pi(\nu-n)}{\sin \pi(\nu-n)} . \\
& \left\{\frac{x^{-\nu} \frac{\Gamma(\alpha-\nu) \Gamma(\beta-\nu)}{\Gamma(1-\nu)} F(\alpha-\nu, \beta-\nu, 1-\nu, x)-\frac{\Gamma(\alpha) \Gamma(\beta)}{\Gamma(1+\nu)} F(\alpha, \beta, 1+\nu, x)}{\nu-n}\right\}
\end{aligned}
$$


provided that $O<x<1, \alpha+\beta-\nu \geqq 1$, and $\nu>0$. When $\nu$ approaches the integral value $n$, the second member of (12) becomes indeterminate, for the denominator of the bracket vanishes and so does the numerator, but the indeterminate form can be evaluated; that is when $\nu=n=0$ or a positive integer

$$
\begin{aligned}
y_{1}(x) & =(-1)^{\mathrm{n}}\left\{\frac{d}{d \nu} \frac{x^{-\nu} \Gamma(\alpha-\nu) \Gamma(\beta-\nu) F(\alpha-\nu, \beta-\nu, 1-\nu, x)}{\Gamma(1-\nu)}\right. \\
& \left.-\frac{d}{d \nu} \frac{\Gamma(\alpha) \Gamma(\beta)}{\Gamma(1+\nu)} F(\alpha, \beta, 1+\nu, x)\right\}_{\nu=n} \\
& =(-1)^{\mathrm{n}}\left\{\frac{-\Gamma(\alpha) \Gamma(\beta)}{\Gamma(1+n)} F(\alpha, \beta, 1+n, x) \log x\right. \\
& +x^{-n} \sum_{s=0}^{\infty} \frac{x^{\mathrm{s}}}{\Gamma(s+1)} \frac{d}{d \nu}\left(\frac{\Gamma(s+\alpha-\nu) \Gamma(s+\beta-\nu)}{\Gamma(s+1-\nu)}\right) \\
& \left.-\sum_{s=0}^{\infty} x^{8} \frac{\Gamma(s+\alpha) \Gamma(s+\beta)}{\Gamma(s+1)} \frac{d}{d \nu} \frac{1}{\Gamma(s+1+\nu)}\right\}_{\nu=n} \\
& =(-1)^{n}\left\{-\frac{\Gamma(\alpha) \Gamma(\beta)}{\Gamma(n+1)} F(\alpha, \beta, n+1, x) \log x\right. \\
& +x^{-n} \sum_{s=0}^{\infty} x^{8} \frac{\Gamma(s+\alpha-\nu) \Gamma(s+\beta-\nu)}{\Gamma(s+1) \Gamma(s+1-\nu)} \frac{d}{d \nu} \log \frac{\Gamma(s+\alpha-\nu) \Gamma(s+\beta-\nu)}{\Gamma(s+1-\nu)} \\
& \left.+\sum_{s=0}^{\infty} x^{8} \frac{\Gamma(s+\alpha) \Gamma(s+\beta)}{\Gamma(s+1) \Gamma(s+1+\nu)} \frac{d}{d \nu} \log \Gamma(s+1+\nu)\right\}_{\nu=n}
\end{aligned}
$$

By the use of Gauss's formula

$$
\frac{d}{d z} \log \Gamma(z)=-C+\sum_{t=0}^{\infty}\left(\frac{1}{t+1}-\frac{1}{t+z}\right) \equiv \psi(z)
$$

where

$$
C=0.57721566=\text { Euler's constant }
$$

the limit (13) may be written

$$
\begin{aligned}
& y_{1}(x)=(-1)^{\mathrm{n}}\left\{-\frac{\Gamma(\alpha) \Gamma(\beta)}{\Gamma(n+1)} F(\alpha, \beta, n+1, x) \log x\right. \\
& +x^{-\mathrm{n}} \sum_{s=0}^{\infty} x^{\mathrm{s}(s-\nu+\alpha) \Gamma(s-\nu+\beta)} \frac{\Gamma(s+1) \Gamma(s-\nu+1)}{\Gamma(s-\nu+1)-\psi(s-\nu+\alpha)-\psi(s-\nu+\beta))} \\
& \left.+\sum_{s=0}^{\infty} x^{\beta} \frac{\Gamma(s+\alpha) \Gamma(s+\beta)}{\Gamma(s+1) \Gamma(s+\nu+1)} \psi(s+\nu+1)\right\}_{\nu=n}
\end{aligned}
$$


Now $\operatorname{limit}_{\nu=n}\left(\frac{\psi(s-\nu+1)-\psi(s-\nu+\alpha)-\psi(s-\nu+\beta)}{\Gamma(s-\nu+1)}\right)=$

$$
\begin{array}{ll}
=\frac{C+\sum_{t=8-n}^{\infty}\left(\frac{1}{t+\alpha}+\frac{1}{t+\beta}-\frac{1}{t+1+n-s}-\frac{1}{t+1}\right)}{\Gamma(s-n+1)} & \text { if } s-n \geqq 0 \\
=(-1)^{n-8} \Gamma(n-s) & \text { if } s-n<0
\end{array}
$$

Hence the limit (16) is

$$
\begin{aligned}
y_{1}(x) & =x^{-n} \sum_{s=0}^{n-1} \frac{\Gamma(s-n+\alpha) \Gamma(s-n+\beta) \Gamma(n-s)}{\Gamma(s+1)}(-x)^{\beta} \\
& -(-1)^{n} \frac{\Gamma(\alpha) \Gamma(\beta)}{\Gamma(n+1)} F(\alpha, \beta, n+1, x) \log x \\
& +(-1)^{n} \sum_{s=0}^{\infty} x^{8} \frac{\Gamma(s+\alpha) \Gamma(s+\beta)}{\Gamma(s+1) \Gamma(s+n+1)} \sum_{t=s}^{\infty}\left(\frac{1}{t+\alpha}+\frac{1}{t+\beta}\right. \\
& \left.-\frac{1}{t+1+n}-\frac{1}{t+1}\right)
\end{aligned}
$$

This is the value of the second member of (12) when $\nu=n$. If we let $\gamma=\alpha+\beta-\nu$, then the identity (12) may be written in the general form

$$
\begin{aligned}
& \frac{\Gamma(\alpha) \Gamma(\beta)}{\Gamma(\gamma)} F(\alpha, \beta, \gamma, 1-x)= \\
& \quad=\frac{\pi}{\sin \pi(\alpha+\beta-\gamma) \Gamma(\gamma-\alpha) \Gamma(\gamma-\beta)}\left\{\frac{x^{\gamma-\alpha-\beta} \Gamma(\gamma-\alpha) \Gamma(\gamma-\beta)}{\Gamma(1+\gamma-\alpha-\beta)} F(\gamma\right. \\
& \quad-\alpha, \gamma-\beta, 1+\gamma-\alpha-\beta, x) \\
& \left.\quad-\frac{\Gamma(\alpha) \Gamma(\beta)}{\Gamma(1-[\gamma-\alpha-\beta])} F(\alpha, \beta, 1-(\gamma-\alpha-\beta), x)\right\}
\end{aligned}
$$

when $\gamma-\alpha-\beta=-n=$ a negative integer or zero this takes the form $\frac{\Gamma(\alpha) \Gamma(\beta)}{\Gamma(\gamma)} F(\alpha, \beta, \gamma, 1-x)=$

$=\frac{(-1)^{\gamma-\alpha-\beta}}{\Gamma(\gamma-\alpha) \Gamma(\gamma-\beta)}\left\{(-x)^{\gamma-\alpha-\beta} \sum_{s=0}^{s=\alpha+\beta-\gamma-1 \geqq 0} \frac{\Gamma(s+\gamma-\alpha) \Gamma(s+\gamma-\beta) \Gamma(\alpha+\beta-\gamma-s)}{\Gamma(s+1)}(-x)^{s}\right.$ $-\frac{\Gamma(\alpha) \Gamma(\beta)}{\Gamma(\alpha+\beta-\gamma+1)} F(\alpha, \beta, \alpha+\beta-\gamma+1, x) \log x$

$\left.+\sum_{s=0}^{\infty} \frac{\Gamma(s+\alpha) \Gamma(s+\beta) x^{\mathrm{s}}}{\Gamma(s+1) \Gamma(s+\alpha+\beta-\gamma+1)} T_{s}(\alpha, \beta, \gamma)\right\}$ 
where

$$
\mathrm{T}_{\mathrm{s}}(\alpha, \beta, \gamma)=\sum_{t=s}^{\infty}\left(\frac{1}{t+\alpha}+\frac{1}{t+\beta}-\frac{1}{t+1+\alpha+\beta-\gamma}-\frac{1}{t+1}\right)
$$

In case $\alpha+\beta-\gamma=0$ the first summation on the right of (18a) disappears and the principal term, when $x$ is small, contains $\log x$ as a factor. The identity (18) was derived on the assumption that $\gamma \geqq 1$. This restriction can probably be removed to the extent that (18) is an identity in all cases in which both sides of equation (18) have a meaning. We are here interested in the special case of (18a) where

$$
\left.\begin{array}{l}
\alpha=n+\frac{1}{2} \\
\beta=n+\frac{1}{2}+k \\
\gamma=k+1
\end{array}\right\} \begin{gathered}
n+1=\text { a positive integer } \\
\text { but } k \text { need not be } \\
\text { an integer }
\end{gathered}
$$

In this case $\gamma-\alpha-\beta=-2 n$, so that one obtains from (18a) the formula

$$
\begin{gathered}
\frac{(-1)^{\mathrm{n}} \Gamma\left(n+k+\frac{1}{2}\right)}{\Gamma(k+1)} F\left(n+\frac{1}{2}, n+\frac{1}{2}+k, k+1,1-x\right)= \\
=(-1)^{\mathrm{n}} \Gamma\left(n+\frac{1}{2}-k\right)\left\{\sum_{s=1}^{\mathrm{n}} \frac{\Gamma(2 s)}{\Gamma(2[n-s]+1) \Gamma\left(2 s-n+\frac{1}{2}\right) \Gamma\left(2 s-n-k+\frac{1}{2}\right) x^{2 \mathrm{e}}}\right. \\
\left.-x \sum_{s=1}^{\mathrm{n}} \frac{\Gamma(2 s-1)}{\Gamma(2[n-s]+2) \Gamma\left(2 s-n-\frac{1}{2}\right) \Gamma\left(2 s-n-k-\frac{1}{2}\right)} \frac{1}{x^{28}}\right\} \\
-\frac{\log x}{\pi \Gamma\left(k-n+\frac{1}{2}\right)} \cdot \sum_{s=0}^{\infty} \frac{\Gamma\left(s+n+\frac{1}{2}\right) \Gamma\left(s+n+\frac{1}{2}+k\right)}{\Gamma(s+1) \Gamma(s+2 n+1)} x^{s} \\
+\frac{1}{\pi \Gamma\left(k-n+\frac{1}{2}\right)} \sum_{s=0}^{\infty} \frac{\Gamma\left(s+n+\frac{1}{2}\right) \Gamma\left(s+n+\frac{1}{2}+k\right)}{\Gamma(s+1) \Gamma(s+2 n+1)} x^{s} T_{\mathrm{s}}(n, k)
\end{gathered}
$$

where

$$
T_{\mathrm{B}}(n, k) \equiv \sum_{t=s}^{\infty}\left(\frac{1}{t+n+\frac{1}{2}}+\frac{1}{t+n+k+\frac{1}{2}}-\frac{1}{t+2 n+1}-\frac{1}{t+1}\right)
$$


In particular

$$
\begin{gathered}
T_{0}(o, o)=4 \log 2 \\
T_{0}(n, k)=T_{0}(o, o)-\sum_{t=0}^{\mathrm{n}-1} \frac{1}{t+\frac{1}{2}}-\sum_{t=0}^{\mathrm{n}-1+\mathrm{k}} \frac{1}{t+\frac{1}{2}}+\sum_{t=0}^{2 \mathrm{n}-1} \frac{1}{t+1}
\end{gathered}
$$

if $k$ is integral

There is also the general recurrence relation

$$
T_{\mathrm{B}+1}(n k)=T_{\mathrm{B}}(n k)-\frac{1}{s+n+\frac{1}{2}}-\frac{1}{s+n+k+\frac{1}{2}}+\frac{1}{s+2 n+1}+\frac{1}{s+1}
$$

The special case of (21) where $n=0$ is

$$
\begin{gathered}
\frac{\Gamma\left(k+\frac{1}{2}\right)}{\Gamma(k+1)} F\left(\frac{1}{2}, \frac{1}{2}+k, k+1,1-x\right)=-\frac{1}{\sqrt{\pi}} F\left(\frac{1}{2}, \frac{1}{2}+k, 1, x\right) \log x \\
+\frac{1}{\pi} \frac{1}{\Gamma\left(\frac{1}{2}+k\right)} \sum_{s=0}^{\infty} \frac{\Gamma\left(s+\frac{1}{2}\right) \Gamma\left(s+\frac{1}{2}+k\right)}{[\Gamma(s+1)]^{2}} x^{s} \sum_{t=s}^{\infty}\left(\frac{1}{t+\frac{1}{2}}+\frac{1}{t+\frac{1}{2}+k}-\frac{2}{t+1}\right)
\end{gathered}
$$

The special case of (21) where $k=0$ is

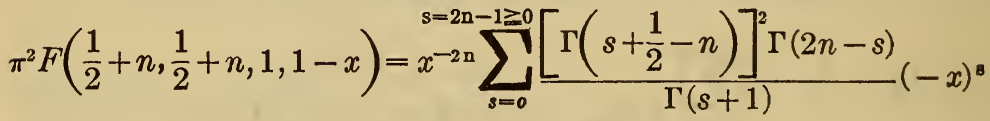

$$
\begin{aligned}
& -\frac{\sqrt{\pi} \Gamma\left(n+\frac{1}{2}\right)}{2^{2 n} \Gamma(n+1)} F\left(\frac{1}{2}+n, \frac{1}{2}+n, 2 n+1, x\right) \log x \\
& +\sum_{s=0}^{\infty} \frac{\left[\Gamma\left(s+\frac{1}{2}+n\right)\right]^{2} x^{8}}{\Gamma(s+1) \Gamma(s+2 n+1)} \sum_{t=8}^{\infty}\left(\frac{2}{t+n+\frac{1}{2}}-\frac{1}{t+2 n+1}-\frac{1}{t+1}\right)
\end{aligned}
$$

The special case where $k=n=0$ is

$$
\begin{aligned}
& \pi F\left(\frac{1}{2}, \frac{1}{2}, 1,1-x\right)=-F\left(\frac{1}{2}, \frac{1}{2}, 1, x\right) \log x \\
& +\frac{1}{\pi} \sum_{s=0}^{\infty}\left[\frac{\Gamma\left(s+\frac{1}{2}\right)}{\Gamma(s+1)}\right]^{2} x^{s} \sum_{t=s}^{\infty} \frac{1}{(t+1)\left(t+\frac{1}{2}\right)}
\end{aligned}
$$

A special case of (18a) is $\alpha=\beta=n+\frac{1}{2}, \gamma=2 n+1$. 
This gives

$$
\begin{aligned}
& \frac{1}{\sqrt{\pi}} \cdot \frac{1}{2^{2 \mathrm{n}+1}} \frac{\Gamma\left(n+\frac{1}{2}\right)}{\Gamma(n+1)} F\left(n+\frac{1}{2}, n+\frac{1}{2}, 2 n+1,1-x\right)= \\
& =\frac{1}{2 \pi}\left\{-F\left(n+\frac{1}{2}, n+\frac{1}{2}, 1, x\right) \log x\right. \\
& \left.+\frac{1}{\left[\Gamma\left(n+\frac{1}{2}\right)\right]^{2}} \sum_{s=0}^{\infty} x^{8}\left[\frac{\Gamma\left(s+n+\frac{1}{2}\right)}{\Gamma(s+1)}\right]^{2} T_{\mathrm{B}}(n)\right\}
\end{aligned}
$$

where

$$
\begin{aligned}
& T_{0}(0)=4 \log 2, T_{0}(n)=4 \log 2-2 \sum_{t=0}^{n-1} \frac{1}{t+\frac{1}{2}} \text { if } n>0 \\
& T_{\mathrm{B}}(n)=T_{0}(n)+2 \sum_{t=0}^{8-1}\left(\frac{1}{t+1}-\frac{1}{t+n+\frac{1}{2}}\right) \text { if } s>0
\end{aligned}
$$

Appendix 2.-GRAPHICAL METHOD OF COMPUTING $B_{0}$ In the integral

let

$$
\frac{p^{2}}{\pi a} \int_{0}^{l} d \lambda_{1} \int_{0}^{\lambda_{1}} d \lambda \frac{K\left(\frac{1}{\sqrt{1+\frac{\lambda_{2}}{4 a^{2}}}}\right)}{\sqrt{1+\frac{\lambda^{2}}{4 a^{2}}}}
$$

This becomes

$$
\eta_{1}=\frac{\lambda}{2 a} \text { and } \eta=\frac{l}{2 a}
$$

$$
\frac{4 a^{3}}{\pi} \frac{p^{2}}{a^{2}} B_{0} \text { where } B_{0}=\int_{0}^{\eta} d \eta_{2} \int_{0}^{\eta_{1}} d \eta_{1} \frac{K\left(\frac{1}{\sqrt{1+\eta_{1}{ }^{2}}}\right)}{\sqrt{1+\eta_{1}{ }^{2}}}
$$

This function $B_{0}$ of $\eta$ has been evaluated by means of an integraph and plotted as a function of $k^{2}=\frac{1}{1+\eta^{2}}$ in Figure 2 .

Appendix 3.-EVALUATION OF $\sqrt{r_{1} r_{2}} \int_{0}^{l} d \lambda_{1} \int_{0}^{\lambda_{1}} \phi_{1}(\mu) d \lambda$ TO SECOND ORDER Here $\mu$ is a function of $\lambda$ defined by

$$
\mu^{2}=\frac{1}{1+\frac{\lambda^{2}+\left(r_{2}-r_{1}\right)^{2}}{4 r_{1} r_{2}}}
$$


and $\phi_{1}(\mu)$ is one of the functions defined by (38) of the text. By inverting the order of integration

$$
\sqrt{r_{1} r_{2}} \int_{0}^{l} d \lambda_{1} \int_{0}^{\lambda_{1}} d \lambda \phi_{1}(\mu)=l \sqrt{r_{1} r_{2}} \int_{0}^{l} \phi_{1}(\mu) d \lambda-\sqrt{r_{1} r_{2}} \int_{0}^{l} \phi_{1}(\mu) \lambda d \lambda
$$

But by (1)

$$
\lambda d \lambda=-4 r_{1} r_{2} \frac{d \mu}{\mu^{3}}
$$

so that

$$
-\sqrt{r_{1} r_{2}} \int_{0}^{l} \phi_{1}(\mu) \lambda d \lambda=-4\left(r_{1} r_{2}\right)^{3 / 3} \int_{\mu_{1}-\frac{k^{2}\left(\xi-\xi^{1}\right)}{8}}^{\mu_{1}} \phi^{2}(\mu)
$$

where

$$
\begin{aligned}
k^{2} & =\frac{1}{1+\frac{l^{2}}{4 a^{2}}} \quad \text { and } \xi=\frac{r_{2}-a}{a}, \xi^{1}=\frac{r_{1}-a}{a} \\
\mu_{1} & =\frac{1}{\sqrt{1+\frac{l^{2}}{4 r_{1} r_{2}}}}=k\left\{1+\frac{1-k^{2}}{2}\left(\xi+\xi^{1}\right)\right. \\
& \left.-\frac{3}{2} k^{2}\left(1-k^{2}\right) \xi \xi^{1}-\left(1-k^{2}\right)\left(1+3 k^{2}\right) \frac{\left(\xi-\xi^{1}\right)^{2}}{8}\right\} \\
\mu_{2} & =\frac{1}{\sqrt{1+\frac{\left(r_{2}-r_{1}\right)^{2}}{4 r_{1} r_{2}}}}=1-\frac{\left(\xi-\xi^{1}\right)^{2}}{8}
\end{aligned}
$$

Making use of the integral formula (49) gives

$$
\begin{aligned}
& -\sqrt{r_{1} r_{2}} \int_{0}^{l} \phi_{1}(\mu) \lambda d \lambda=-4\left(r_{1} r_{2}\right)^{3 / 2} \int_{\mu_{1}-\frac{k^{3}\left(\xi-\xi^{1}\right)^{2}}{8}}^{\mu_{2}} \phi(\mu) \\
& =\frac{4\left(r_{1} r_{2}\right)^{3 / 2}}{3}\left\{\left[\frac{1-\mu^{2}}{\mu} \frac{d \phi_{1}(\mu)}{d \mu}\right]_{\mu=\mu_{1}-\frac{k^{3}\left(\xi-\xi^{1}\right)}{8}}-\frac{1-\mu_{2}^{2}}{\mu_{2}} \frac{d \phi_{1}\left(\mu_{2}\right)}{d \mu_{2}}\right\}
\end{aligned}
$$

Now by (4) $1-\mu_{2}{ }^{2}=\frac{\left(\xi-\xi^{1}\right)^{2}}{4}$, hence by (55) and (56)

$$
\begin{gathered}
-\left(\frac{1-\mu_{2}{ }^{2}}{\mu_{2}}\right) \frac{d \phi_{1}\left(\mu_{2}\right)}{d \mu_{2}}=\frac{1}{\pi}\left\{-1-\frac{3}{8}\left(\xi-\xi^{1}\right)^{2} \log \left(\xi-\xi^{1}\right)\right. \\
\left.+\frac{3}{16}(6 \log 2-3)\left(\xi-\xi^{1}\right)^{2}\right\}
\end{gathered}
$$


Also by Maclaurin's theorem

$$
\begin{aligned}
& {\left[\left(\frac{1-\mu^{2}}{\mu}\right) \frac{d \phi_{1}(\mu)}{d \mu}\right]_{\mu=\mu_{1}-\frac{k^{3}\left(\xi-\xi^{1}\right)}{8}}=\frac{1-\mu_{1}^{2}}{\mu_{1}} \frac{d \phi_{1}\left(\mu_{1}\right)}{d \mu_{1}}} \\
& -\frac{k^{3}\left(\xi-\xi^{1}\right)^{2}}{8} \frac{d}{d \mu_{1}}\left[\frac{1-\mu_{1}{ }^{2}}{\mu_{1}} \frac{d \phi_{1}\left(\mu_{1}\right)}{d \mu_{1}}\right] \\
& =\frac{1}{2}\left[\phi_{0}\left(\mu_{1}\right)-\left(\frac{2}{\mu_{1}^{2}}-1\right) \phi_{1}\left(\mu_{1}\right)\right]-\frac{3 k^{3}\left(\xi-\xi^{1}\right)^{2}}{8 \mu_{1}{ }^{3}} \phi_{1}\left(\mu_{1}\right)
\end{aligned}
$$

by (47) and (42). Or by (45) and (46)

$$
\begin{gathered}
{\left[\left(\frac{1-\mu^{2}}{\mu}\right) \frac{d \phi_{1}(\mu)}{d \mu}\right]_{\mu=\mu_{1}-\frac{k^{3}\left(\xi-\xi^{1}\right)^{2}}{8}}=\frac{1}{\pi \mu_{1}^{3}}\left[\left(2-\mu_{1}^{2}\right) E\left(\mu_{1}\right)-2\left(1-\mu_{1}^{2}\right) K\left(\mu_{1}\right)\right]} \\
-\frac{3}{8 \pi k_{0}}\left[\left(2-k^{2}\right) K(k)-2 E(k)\right]\left(\xi-\xi^{1}\right)^{2}
\end{gathered}
$$

By means of (6) and (7), the value of (5) becomes

$$
\begin{aligned}
& -\sqrt{r_{1} r_{2}} \int_{0}^{l} \phi_{1}(\mu) \lambda d \lambda \\
& =\frac{4\left(r_{1} r_{2}\right)^{3 / 2}}{\pi}\left\{\frac{\left(2-\mu_{1}^{2}\right) E\left(\mu_{1}\right)-2\left(1-\mu_{1}^{2}\right) K\left(\mu_{1}\right)}{3 \mu_{1}^{3}}-\frac{1}{3}-\frac{1}{8}\left(\xi-\xi^{1}\right)^{2} \log \left(\xi-\xi^{1}\right)\right. \\
& \left.+\frac{1}{8}\left[3 \log 2-\frac{3}{2}+\frac{2 E(k)-\left(2-k^{2}\right) K(k)}{k}\right]\left(\xi-\xi^{1}\right)^{2}\right\}
\end{aligned}
$$

This gives the last term in (2). To evaluate the first, we may make use of the form for $\phi_{1}(\mu)$ given by (41), by which

$$
l \sqrt{r_{1} r_{2}} \int_{0}^{l} \phi_{1}(\mu) d \lambda=\frac{4\left(r_{1} r_{2}\right)^{3 / 2}}{\pi} \eta \int^{\frac{\pi}{2}} \cos 2 \theta d \theta \int_{l}^{\eta} \frac{d \lambda}{\sqrt{\lambda^{2}+\alpha^{2}+\sin ^{2} \theta}}
$$

where

$$
\eta=\frac{l}{2 \sqrt{r_{1} r_{2}}}=\frac{\sqrt{1-\mu_{1}^{2}}}{\mu_{1}} \text {. and } \alpha^{2}=\frac{\left(r_{2}-r_{1}\right)^{2}}{4 r_{1} r_{2}}
$$

Performing the integration with respect to $\lambda$ in (9) gives

$$
\begin{gathered}
l \sqrt{r_{1} r_{2}} \int_{0}^{l} \phi_{1}(\mu) d \lambda=\frac{4\left(r_{1} r_{2}\right)^{3 / 2}}{\pi} \eta \int_{0}^{\frac{\pi}{2}} \cos 2 \theta d \theta\left\{\log \left(\eta+\sqrt{\eta^{2}+\alpha^{2}+\sin ^{2} \theta}\right)\right. \\
\left.-\log \sqrt{\alpha^{2}+\sin ^{2} \theta}\right\}
\end{gathered}
$$

In this integral the term $\log \left(\eta+\sqrt{\eta^{2}+\alpha^{2}+\sin ^{2} \theta}\right)$ may be expanded by Maclaurin's theorem in ascending powers of $\alpha^{2}$, since $\eta$ is finite. (It may be noted that this procedure is not valid if $\eta$ is also infinitesimal. Hence, we can not expect the expression thus obtained to reduce to 
the correct value if we later let $\eta$ approach zero.) This gives for finite values of $\eta$

$$
\begin{aligned}
l \sqrt{r_{1} r_{2}} \int_{0}^{l} \phi_{1}(\mu) d \lambda & =\frac{\left(4 r_{1} r_{2}\right)^{3 / 2}}{\pi} \eta \int_{0}^{\frac{\pi}{2}} \cos 2 \theta d \theta\left\{\left[\log \left(\eta+\sqrt{\eta^{2}+\sin ^{2} \theta}\right)\right.\right. \\
& +\frac{\alpha^{2}}{2}\left[\frac{-\log \sin \theta]}{\sqrt{\eta^{2}+\sin ^{2} \theta}\left(\sqrt{\left.\eta^{2}+\sin ^{2} \theta+\eta\right)}\right.}\right] \\
& \left.+\left[\log \sin \theta-\log \sqrt{\alpha^{2}+\sin ^{2} \theta}\right]\right\}
\end{aligned}
$$

We shall next evaluate the three separate integrals corresponding to the three square brackets on the right of (12).

(A)

$$
\frac{1}{\pi} \int_{0}^{\frac{\pi}{2}} \cos 2 \theta d \theta\left[\log \left(\eta+\sqrt{\eta^{2}+\sin ^{2} \theta}\right)-\log \sin \theta\right]
$$

This is the result of placing $\alpha=0$ in the original integral (11) and evidently reduces to $\int_{\mu_{1}}^{1} \frac{\phi_{1}(\mu) d \mu}{\mu^{2} \sqrt{1-\mu^{2}}}$. Hence, by the use of (52)

(B)

$$
\begin{aligned}
& \frac{1}{\pi} \int_{0}^{\frac{\pi}{2}} \cos 2 \theta d \theta\left[\log \left(\eta+\sqrt{\eta^{2}+\sin ^{2} \theta}\right)-\log \sin \theta\right]= \\
& =\frac{\sqrt{1-\mu_{1}^{2}}}{\pi \mu_{1}^{2}}\left[K\left(\mu_{1}\right)-E\left(\mu_{1}\right)\right] \\
& \frac{\alpha^{2}}{2 \pi} \int_{0}^{\frac{\pi}{2}} \frac{\cos 2 \theta d \theta}{\sqrt{\eta^{2}+\sin ^{2} \theta}\left(\sqrt{\eta^{2}+\sin ^{2} \theta}+\eta\right)} \\
& =\frac{\alpha^{2}}{2 \pi \eta^{2}} \int_{0}^{\frac{\pi}{2}} \frac{\cos 2 \theta d \theta}{\sqrt{1+\frac{\sin ^{2} \theta}{\eta^{2}}\left(1+\sqrt{1+\frac{\sin ^{2} \theta}{\eta^{2}}}\right)}} \\
& =-\frac{a^{2}}{2 \pi} \frac{1}{\sqrt{\pi}} \sum_{n=1}^{\infty} \frac{\Gamma\left(n+\frac{1}{2}\right)}{\Gamma(n+1)} \frac{(-1)^{n}}{\eta^{2 n}}\left\{\int_{0}^{\frac{\pi}{2}} \sin ^{2 n-2} \theta d \theta-2 \int_{0}^{\frac{\pi}{2}} \sin ^{2 n} \theta d \theta\right\} \\
& =\frac{a^{2}}{2}\left\{-\frac{1}{2 \pi} \sum_{n=1}^{\infty} \frac{\Gamma\left(n-\frac{1}{2}\right) \Gamma\left(n+\frac{1}{2}\right)}{\Gamma(n) \Gamma(n+1)}\left(-\frac{1}{\eta^{2}}\right)^{n}\right. \\
& \left.+\frac{1}{\pi} \sum_{n=1}^{\infty}\left[\frac{\Gamma\left(n+\frac{1}{2}\right)}{\Gamma(n+1)}\right]^{2}\left(\frac{-1}{\eta^{2}}\right)^{n}\right\} \\
& =\frac{\alpha^{2}}{2}\left\{-1+F\left(\frac{1}{2}, \frac{1}{2}, 1-\frac{1}{\eta^{2}}\right)+\frac{1}{4 \eta^{2}} F\left(\frac{1}{2}, \frac{3}{2}, 2,-\frac{1}{\eta^{2}}\right)\right\} \\
& =\frac{\alpha^{2}}{2}\left\{-1+\sqrt{1-k^{2}}\left[F\left(\frac{1}{2}, \frac{1}{2}, 1, k^{2}\right)+\frac{k^{2}}{4} F\left(\frac{3}{2}, \frac{3}{2}, 2 k^{2}\right)\right]\right\}
\end{aligned}
$$


(by Euler's transformation)

$$
\begin{aligned}
& =\frac{\alpha^{2}}{2}\left\{-1+\sqrt{1-k^{2}}\left[F\left(\frac{1}{2}, \frac{1}{2}, 1, k^{2}\right)+\frac{k d}{2 d k} F\left(\frac{1}{2}, \frac{1}{2}, 1, k^{2}\right)\right]\right\} \\
& =\frac{\alpha^{2}}{\pi}\left\{-\frac{\pi}{2}+\sqrt{1-k^{2}}\left(K(k)+\frac{k}{2} \frac{d}{d k} K(k)\right)\right\} \\
& =\frac{\alpha^{2}}{2 \pi}\left\{-\pi+\sqrt{1-k^{2}}\left[K(k)+\frac{E(k)}{1-k^{2}}\right]\right\}
\end{aligned}
$$

This result is, in fact, valid for all positive values of $k$ less than 1 ; that is, for any positive value of $\eta$.

(C)

$$
\frac{1}{\pi} \int_{0}^{\frac{\pi}{2}} \cos 2 \theta d \theta\left[\log \sin \theta-\log \sqrt{\alpha^{2}+\sin ^{2} \theta}\right]
$$

Consider the integral

$$
\begin{aligned}
& -\frac{1}{\pi} \int_{0}^{\frac{\pi}{2}} \cos 2 \theta d \theta \log \sqrt{\alpha^{2}+\sin ^{2} \theta}=-\frac{1}{4 \pi} \int_{0}^{\pi} \cos \theta \log \left(1+2 \alpha^{2}-\cos \theta\right) d \theta \\
& =-\frac{1}{4 \pi} \int_{0}^{\pi} d \theta \cos \theta \log \left(1-\frac{\cos \theta}{1+2 \alpha^{2}}\right)=\frac{1}{4 \pi} \sum_{n=1}^{\infty} \frac{1}{n\left(1+2 \alpha^{2}\right)^{\mathrm{n}}} \int_{0}^{\pi} \cos ^{\mathrm{n}+1} \theta d \theta \\
& =\frac{\left(1+2 \alpha^{2}\right)}{8 \sqrt{\pi}} \sum_{k=0}^{\infty} \frac{\Gamma\left(k+\frac{1}{2}\right)}{\Gamma(k+2)} \frac{1}{\left(1+2 \alpha^{2}\right)^{2 \mathbf{k}+2}} \\
& =-\frac{\left(1+2 \alpha^{2}\right)}{4}\left[-\frac{1}{2 \sqrt{\pi}} \sum_{s=1}^{\infty} \frac{\Gamma\left(s-\frac{1}{2}\right)}{\Gamma(s+1)}, \frac{1}{\left(1+2 \alpha^{2}\right)^{28}}\right] \\
& =\frac{\left(1+2 \alpha^{2}\right)}{4}\left\{1-\sqrt{1-\frac{1}{\left(1+2 \alpha^{2}\right)^{2}}}\right\}=\left[\frac{\sqrt{1+\alpha^{2}}-\alpha}{2}\right]^{2} \text { if } \alpha \geqq 0
\end{aligned}
$$

Placing $\alpha=o$ gives $\frac{1}{\pi} \int_{0}^{\frac{\pi}{2}} \cos 2 \theta \log \sin \theta d \theta=-\frac{1}{4}$

Hence

$$
\begin{aligned}
& \frac{1}{\pi} \int_{0}^{\frac{\pi}{2}} \cos 2 \theta\left[\log \sin \theta-\log \sqrt{\alpha^{2}+\sin ^{2} \theta}\right] d \theta=\frac{\alpha}{2}\left(\alpha-\sqrt{1+\alpha^{2}}\right) \\
& \quad=-\frac{\alpha}{2}+\frac{\alpha^{2}}{2}+\ldots .
\end{aligned}
$$

where

$$
\alpha=\frac{r_{2}-r_{1}}{2 \sqrt{r_{1} r_{2}}} \geqq 0
$$


The second order term $\frac{\alpha^{2}}{2}$ may be replaced by $\frac{\left(\xi-\xi^{1}\right)^{2}}{8}$, but the first order term is

$$
-\frac{\alpha}{2}=-\left(\frac{r_{2}-r_{1}}{4 \sqrt{r_{1} r_{2}}}\right)=-\frac{\xi-\xi^{1}}{4(1+\xi)^{\frac{1}{2}}\left(1+\xi^{1}\right)^{\frac{1}{3}}}=-\left(\frac{\xi-\xi^{1}}{4}\right)+\frac{\xi^{2}-\xi^{12}}{8} \ldots .
$$

provided that $\xi-\xi^{1}>0$, as is assumed throughout. Hence, collecting the results (13), (14), and (15) gives for the value of (12) after placing $\eta=\frac{\sqrt{1-\mu_{1}^{2}}}{\mu_{1}}$

$$
\begin{gathered}
l \sqrt{r_{1} r_{2}} \int_{0}^{l} \phi_{1}(\mu) d \mu \\
=\frac{4\left(r_{1} r_{2}\right)^{8 / 3}}{\pi}\left\{\frac{1-\mu_{1}^{2}}{\mu_{1}^{3}}\left(K\left(\mu_{1}\right)-E\left(\mu_{1}\right)\right)-\frac{\pi \sqrt{1-k^{2}}}{4 k}\left(\xi-\xi^{1}\right)\right. \\
\left.+\frac{\pi \sqrt{1-k^{2}}}{4 k}\left(\xi^{2}-\xi^{1^{2}}\right)+\frac{\left(\xi-\xi^{1}\right)^{2}}{8 k}\left[E(k)+\left(1-k^{2}\right) K(k)\right]\right\}
\end{gathered}
$$

This is the first term of the right side of (2). Adding (16) and (8) gives for (2)

$$
\begin{aligned}
& \sqrt{r_{1} r_{2}} \int_{0}^{l} d \lambda_{1} \int_{0}^{\lambda_{1}} d \lambda \phi_{1}(\mu) \\
& =\frac{4\left(r_{1} r_{2}\right)^{3 / 2}}{\pi}\left\{\frac{1}{3}\left[\frac{\left(1-\mu_{1}^{2}\right) K\left(\mu_{1}\right)-\left(1-2 \mu_{1}^{2}\right) E\left(\mu_{1}\right)}{\mu_{1}^{3}}-1\right]-\frac{\pi \sqrt{1-k^{2}}}{4 k}\left(\xi-\xi^{1}\right)\right. \\
& -\frac{\left(\xi-\xi^{1}\right)^{2}}{8} \log \left(\xi-\xi^{1}\right)+\frac{\pi \sqrt{1-k^{2}}}{4 k}\left(\xi^{2}-\xi^{1^{2}}\right) \\
& \left.+\frac{\left(\xi-\xi^{1}\right)^{2}}{8}\left[3 \log 2-\frac{3}{2}+\frac{3 E(k)-K(k)}{k}\right]\right\}
\end{aligned}
$$

The first part of this expression which depends upon $\mu_{1}$ must next be simplified by expressing $\mu_{1}$ in terms of $k$ and infinitesimals, according to (3).

To do this let $\left\{\begin{array}{l}\epsilon_{1}=\frac{k\left(1-k^{2}\right)}{2}\left(\xi+\xi^{1}\right) \\ \epsilon_{2}{ }^{2}=\frac{3}{2} k^{3}\left(1-k^{2}\right) \xi \xi^{1}+k\left(1-k^{2}\right)\left(1+3 k^{2}\right) \frac{\left(\xi-\xi^{1}\right)^{2}}{8}\end{array}\right.$

Then

$$
\mu_{1}=k+\epsilon_{1}-\epsilon_{2}{ }^{2}
$$

Let

$$
F(k)=\frac{\left(1-k^{2}\right) K(k)-\left(1-2 k^{2}\right) E(k)}{3 k^{3}}
$$


Then

$$
F^{\prime}(k)=\frac{E(k)-K(k)}{k^{4}} \text { and } F^{\prime \prime}(k)=\frac{4}{k^{5}}\left[K(k)-\frac{1-\frac{3 k^{2}}{4}}{1-k^{2}} E(k)\right]
$$

Then to the second order inclusive

$$
\begin{aligned}
F\left(\mu_{1}\right) & =F(k)+\epsilon_{1} F^{\prime}(k)-\epsilon_{2}{ }^{2} F^{\prime}(k)+\frac{\epsilon_{1}{ }^{2}}{2} F^{\prime \prime}(k) \\
& =\frac{\left(1-k^{2}\right) K(k)-\left(1-2 k^{2}\right) E(k)}{3 k^{2}}-\left(\frac{\left(1-k^{2}\right)}{2}\right)\left(\frac{K(k)-E(k)}{k^{3}}\right)\left(\xi+\xi^{1}\right) \\
& +2\left(\frac{1-k^{2}}{k^{3}}\right)\left[\left(1-\frac{k^{2}}{4}\right) K(k)-E(k)\right] \xi \xi^{1} \\
& +\frac{5}{8}\left(\frac{1-k^{2}}{k^{3}}\right)\left[\left(1-\frac{k^{2}}{5}\right) K(k)-E(k)\right]\left(\xi-\xi^{1}\right)^{2}
\end{aligned}
$$

Inserting these values in (17) gives

$$
\begin{aligned}
& \sqrt{r_{1} r_{2}} \int_{0}^{l} d \lambda_{1} \int_{0}^{\lambda_{1}} \phi_{1}(\mu) d \lambda \\
&=\frac{4\left(r_{1} r_{2}\right)^{2 / 3}}{\pi}\left\{\frac{1}{3}\left[\frac{\left(1-k^{2}\right) K-\left(1-2 k^{2}\right) E}{k^{3}}-1\right]-\frac{\left(1-k^{2}\right)(K-E)}{2 k^{3}}\left(\xi+\xi^{1}\right)\right. \\
&-\frac{\pi \sqrt{1-k^{2}}}{4 k}\left(\xi-\xi^{1}\right)+\frac{\pi \sqrt{1-k^{2}}}{4 k}\left(\xi^{2}-\xi^{1^{2}}\right) \\
&+\frac{2\left(1-k^{2}\right)\left[\left(1-\frac{k^{2}}{4}\right) K-E\right]}{k^{3}} \xi^{1} \\
&+\frac{5}{8}\left[\frac{\left(1-\frac{7}{5} k^{2}+\frac{k^{4}}{5}\right) K-\left(1-\frac{8 k^{2}}{5}\right) E}{k^{3}}-\frac{3}{10}\right]\left(\xi-\xi^{1}\right)^{2} \\
&\left.-\frac{\left(\xi-\xi^{1}\right)^{2}}{8} \log \left(\frac{\xi-\xi^{1}}{8}\right)\right\}
\end{aligned}
$$

where the modulus of $K$ and $E$ is understood to be $k$. The factor of the expression on the right is

$$
\left(r_{1} r_{2}\right)^{8 / 2}=a^{3}(1+\xi)^{3 / 2}\left(1+\xi^{1}\right)^{3 / 2}=a^{3}\left[1+\frac{3}{2}\left(\xi+\xi^{1}\right)+3 \xi \xi^{1}+\frac{3}{8}\left(\xi-\xi^{1}\right)^{2} \ldots .\right]
$$


The final simplification of (18) is obtained by multiplying in this factor and rearranging the result. This may be written

$$
\begin{aligned}
& \sqrt{r_{1} r_{2}} \int_{0}^{l} d \lambda_{1} \int_{0}^{\lambda_{1}} d \lambda \phi_{1}(\mu) \\
& =\frac{4 a^{3}}{\pi}\left\{A_{0}+A_{1}\left(\xi+\xi^{1}\right)-\frac{\pi l}{8 a}\left(\xi-\xi^{1}\right)-\frac{\pi l}{1^{\prime} 6 a}\left(\xi^{2}-\xi^{12}\right)+A_{3} \xi \xi^{1}+A_{4}\left(\xi-\xi^{1}\right)^{2}\right. \\
& \left.\quad-\frac{\left(\xi-\xi^{1}\right)^{2}}{8} \log \left(\frac{\xi-\xi^{1}}{8}\right)\right\}
\end{aligned}
$$

where

$$
\left.\begin{array}{l}
A_{0}(k)=\frac{1}{3}\left[\frac{\left(1-k^{2}\right) K-\left(1-2 k^{2}\right) E}{k^{3}}-1\right] \\
A_{1}(k)=\frac{1}{2}\left[\frac{E}{k}-1\right] \\
A_{3}(k)=\frac{2 E-\left(1-k^{2}\right) K}{2 k}-1 \\
A_{4}(k)=\frac{1}{4}\left[\frac{2 E-\left(1-\frac{k^{2}}{2}\right) K}{k}-\frac{5}{4}\right]
\end{array}\right\}
$$

where $K$ and $E$ are the complete elliptic integrals of the first and second kinds, respectively, of modulus $k$ and

$$
k^{2}=\frac{1}{1+\left(\frac{l}{2 a}\right)^{2}}, \xi=\frac{r_{2}-a}{a}, \xi^{1}=\frac{r_{1}-a}{a} \text { and } \xi-\xi^{1}>0
$$

Appendix 4.-SECOND ORDER APPROXIMATION FOR $G_{0}(y)$

$$
G_{0}(y)=-\sqrt{r_{1} r_{2}} \int_{0}^{y} d \lambda_{1} \int_{0}^{\lambda_{1}} d \lambda \phi_{1}(\mu)
$$

Here $y$ is a first order infinitesimal and

$$
1-\mu^{2}=\frac{\lambda^{2}}{4 r_{1} r_{2}}+\frac{\left(r_{2}-r_{1}\right)^{2}}{4 r_{1} r_{2}}
$$

Since this is a repeated integral over an infinitesimal range, the second order approximation will be obtained by writing, according to (56)

$$
\phi_{1}(\mu)=\frac{1}{\pi}\left\{\log 8 a-2-\log \sqrt{\lambda^{2}+\left(r_{2}-r_{\mathrm{c}}\right)^{2}}\right\} \quad \text { +infinitesimals }
$$


Hence to the second order if $z \equiv r_{2}-r_{1}+i y$

$-\sqrt{r_{1} r_{2}} \int_{0}^{y} d \lambda_{1} \int_{0}^{\lambda_{1}} d \lambda \phi_{1}(\mu)=\frac{a}{\pi}\left\{\left(2-\log 8(l) \frac{y^{2}}{2}+\int^{y} d \lambda_{1} \int^{\lambda_{1}} d \lambda \log \sqrt{\lambda^{2}+\left(r_{2}-r_{1}\right)^{2}}\right\}\right.$

or

$$
G_{0}(y)=\frac{4 a^{3}}{\pi}\left\{\frac{y^{2}}{16 a^{2}}+\frac{\left(\xi-\xi^{1}\right)^{2}}{8} \log \left(\frac{\xi-\xi^{1}}{8}\right)-\frac{z^{2}}{8 a^{2}} \log \frac{z}{8 a}\right\}
$$

where the real part is to be understood.

Appendix 5.-EVALUATION OF $G_{1}(y)$ TO THE SECOND ORDER

where

$$
x \equiv r_{2}-r_{1} \quad y \equiv y_{2}-y_{1}
$$

$$
\begin{gathered}
G_{1}(y)=r_{1} r_{2} p^{2} \sum_{n=1}^{\infty} \frac{1}{\sqrt{n^{2}}} \int_{0}^{\infty} \frac{1-\frac{p^{2} s^{2}}{n^{2}}}{\left[1+\frac{p^{2} s^{2}}{n^{2}}\right]^{2}} \epsilon^{-\mathrm{ys}}\left[J_{\mathrm{n}-1}\left(r_{1} s\right) J_{\mathrm{n}-1}\left(r_{2} s\right)\right. \\
\left.+J_{\mathrm{n}+1}\left(r_{1} s\right) J_{\mathrm{n}+1}\left(r_{2} s\right)\right] d s
\end{gathered}
$$

Since $x, y$, and $p$ are to be considered as infinitesimals of the same order, $\frac{x}{p}$ and $\frac{y}{p}$ will be finite ratios in general. This may also be written

$$
G_{1}(y)=\sqrt{r_{1} r_{2}} p^{2} \frac{\partial}{\partial p} p \sum_{n=1}^{\infty} \frac{u_{\mathrm{n}-1}\left(x, y, \frac{p}{n}\right)+u_{\mathrm{n}+1}\left(x, y, \frac{p}{n}\right)}{n^{2}}
$$

where

$$
u_{\mathbf{k}}(x, y, \alpha) \equiv \sqrt{r_{1} r_{2}} \int_{0}^{\infty} \frac{e^{-\mathbf{y s}}}{1+\alpha^{2} s^{2}} J_{\mathbf{k}}\left(r_{1} s\right) J_{\mathbf{k}}\left(r_{2} s\right) d s
$$

Since the second member of (3) contains the second order factor $p^{2}$, we may neglect all terms in $u_{\mathrm{k}}$ which are infinitesimal in $x, y$, and $\alpha$.

To simplify $u_{\mathrm{k}}$ we note that by (61), (62), and (56)

$$
\phi_{\mathbf{k}}(\mu)=\sqrt{r_{1} r_{2}} \int_{0}^{\infty} e^{-\mathrm{ys}} J_{\mathbf{k}}\left(r_{1} s\right) J_{\mathbf{k}}\left(r_{2} s\right) d s=\frac{1}{\pi}\left\{\frac{T_{0}\left(k_{)}\right)}{2}+\log 2 a-\log \sqrt{x^{2}+y^{2}}\right\}
$$

$$
\text { +infinitesimals }
$$

where

$$
\begin{gathered}
\mu^{2}=\frac{1}{1+\frac{x^{2}+y^{2}}{4 r_{1} r_{2}}} \\
T_{0}(0)=4 \log 2, T_{0}(k)=4 \log 2-2 \sum_{t=0}^{k-1} \frac{1}{t+\frac{1}{2}} \text { if } k>0
\end{gathered}
$$


The expression (5) may also be written

where

$$
\phi_{\mathbf{k}}(\mu)=\frac{1}{\pi}\left\{\frac{T_{0}(k)}{2}+\log 2 a-\log z\right\}
$$

$$
z=x+i y
$$

and where the real part only of the complex function in (7) is to be retained. From (4) and (5) it follows that

$$
u_{\mathbf{k}}(x, y, \alpha)-\phi_{\mathbf{k}}(\mu)=-\sqrt{r_{1} r_{2}} \int_{0}^{\infty} \frac{\alpha^{2} s^{2}}{1+\alpha^{2} s^{2}} e^{-\mathrm{y} 8} J_{\mathbf{k}}\left(r_{1} s\right) J_{\mathbf{k}}\left(r_{2} s\right) d s
$$

We may next show that when $\alpha, y$, and $x \cdot\left(\equiv r_{2}-r_{1}\right)$ are positive infinitesimals of the same order, the integral in the second member of (9) differs by an infinitesimal from that obtained by replacing the term $J_{\mathbf{k}}\left(r_{1} s\right) J_{\mathbf{E}}\left(r_{2} s\right)$ by the principal term of its asymptotic expansion; that is, by $\frac{1}{\pi} \frac{\cos x s}{s \sqrt{r_{1} r_{2}}}$. When this is proven to be allowable (9) will reduce to

$$
\begin{aligned}
u_{\mathbf{k}}(x, y, \alpha) & =\phi_{\mathbf{s}}(\mu)-\frac{1}{\pi} \int_{0}^{\infty} \frac{\alpha^{2} s}{1+\alpha^{2} s^{2}} e^{-y 8} \cos x s d s \\
& =\phi_{\mathbf{s}}(\mu)-\frac{1}{\pi} \int_{0}^{\infty} \frac{s}{1+s^{2}} e^{-\frac{\mathrm{y}}{\alpha} \mathrm{s}} \cos \frac{x}{\alpha} s d s \\
& =\frac{1}{\pi}\left\{\frac{T_{0}(k)}{2}-\log \frac{z}{2 a}-\int_{0}^{\infty} \frac{s e^{i \frac{z}{\alpha} \mathrm{s}}}{1+s^{2}} d s\right\}
\end{aligned}
$$

where the imaginary part is to be rejected.

The justification of this procedure is by no means self-evident, for the magnitudes $r_{1} s$ and $r_{2} s$ are not large in the lower part of the range of integration; that is, where as is small. However, in this case the factor $\frac{\alpha^{2} s^{2}}{1+\alpha^{2} s^{2}}$ reduces the integrand to a negligible quantity; when $\alpha$ is not small, $s$ is of the order of $\frac{1}{\alpha}$, and the asymptotic expansions for the Bessel's function then become valid. With this by way of introduction, we may proceed to the proof by writing the second member of (9) in the form

$$
\begin{aligned}
& -\sqrt{r_{1} r_{2}} \int_{0}^{\infty} \frac{\alpha^{2} s^{2}}{1+\alpha^{2} s^{2}} e^{-y 8} J_{\mathbf{k}}\left(r_{1} s\right) J_{\mathbf{k}}\left(r_{2} s\right) d s \\
& =-\sqrt{r_{1} r_{2}} \sqrt{\alpha} \int_{0}^{1} \frac{t^{2}}{1+\alpha t^{2}} e^{-\frac{y}{\alpha} t \sqrt{\alpha}} J_{\mathbf{k}}\left(\frac{r_{1} t}{\sqrt{\alpha}}\right) J_{\mathbf{k}}\left(\frac{r_{2} t}{\sqrt{\alpha}}\right) d t \\
& -\sqrt{r_{1} r_{2}} \sqrt{\alpha} \int_{1}^{\infty} \frac{t^{2}}{1+\alpha t^{2}} e^{-\frac{y}{\alpha} t \sqrt{\alpha}} J_{\mathbf{k}}\left(\frac{r_{1} t}{\sqrt{\alpha}}\right) J_{\mathbf{k}}\left(\frac{r_{2} t}{\sqrt{\alpha}}\right) d t
\end{aligned}
$$


(which is the result of changing the variable of integration by the transformation $\left.s=\frac{t}{\sqrt{\alpha}}\right)$.

The first integral on the right of (11) is infinitesimal, for it is approximately equal to

$$
-\sqrt{r_{1} r_{2}} \sqrt{\alpha} \int_{0}^{1} t^{2} J_{k}\left(\frac{r_{1} t}{\sqrt{\alpha}}\right) J_{k}\left(\frac{r_{2} t}{\sqrt{\alpha}}\right) d t
$$

This integral, which contains the infinitesimal factor $\sqrt{\alpha}$, is not infinite, for the integral oscillates in sign between finite limits and will be finite in amount, even if $r_{1}=r_{2}$, in which case it reduces to

$$
\int_{0}^{1} t^{2}\left[J_{\mathbf{k}}\left(\frac{r_{1} t}{\sqrt{\alpha}}\right)\right]^{2} d t
$$

Although $\left[J_{k}\left(\frac{r_{1} t}{\sqrt{\alpha}}\right)\right]^{2}$ oscillates between zero and a finite positive value a large number of times when $\alpha$ is small, in the range of $t$ from 0 to 1 its integral is finite. Hence, because of the factor $\sqrt{\alpha}$, the first integral on the right side of (11) is negligible. In the second integral where $t \geqq 1$, we may write

$$
\begin{gathered}
J_{\mathbf{k}}\left(\frac{r_{2} t}{\sqrt{\alpha}}\right) J_{\mathbf{k}}\left(\frac{r_{2} t}{\sqrt{\alpha}}\right)=\frac{1}{\pi \sqrt{r_{1} r_{2}} \frac{t}{\sqrt{\alpha}}}\left\{\cos \frac{x t}{\sqrt{\alpha}}+\frac{x}{2}\left(k^{2}-\frac{1}{4}\right) \frac{\sin \frac{x t}{\sqrt{\alpha}}}{\frac{t}{\sqrt{\alpha}}}\right. \\
\left.+(-1)^{\mathbf{k}}\left[\sin \frac{2 t}{\sqrt{\alpha}}+\left(k^{2}-\frac{1}{4}\right) \frac{\sin \frac{2 t}{\sqrt{\alpha}}}{\frac{t}{\sqrt{\alpha}}}\right]\right\}
\end{gathered}
$$

It is evident that the first term of this expansion, namely,

$$
\frac{1}{\pi \frac{t}{\sqrt{\alpha}}} \cos \frac{x t}{\sqrt{\alpha}}
$$

is the only one which will not give an infinitesimal contribution to the integral. For example, the term

$$
\frac{(-1)^{\mathbf{k}}}{\pi} \frac{\sin \frac{2 t}{\sqrt{\alpha}}}{\frac{t}{\sqrt{\alpha}}}
$$


contributes the integral

$$
-\frac{\alpha(-1)^{\mathrm{k}}}{\pi} \int_{1}^{\infty} \frac{e^{-\frac{y}{\alpha} t \sqrt{\alpha}} t \sin \frac{2 t}{\sqrt{\alpha}}}{1+\alpha t^{2}} d x
$$

which is equal to the algebraic sum of the areas of the arches of the curve represented by the integrand. This curve consists of arches whose width is $\pi \sqrt{\alpha}$ (infinitesimal) and their height continually diminishes in magnitude as $t$ increases, while they alternate in sign. The area (and the integral) is therefore smaller than the area of the first loop or arch, which is infinitesimal.

Consequently the second member of (10) reduces to

$$
-\frac{\alpha}{\pi} \int_{1}^{\infty} \frac{t}{1+\alpha t^{2}} e^{-\frac{y}{\alpha} t \sqrt{\alpha}} \cos \left(\frac{x}{\alpha} t \sqrt{\alpha}\right) d t
$$

A further substitution $t=\frac{s}{\sqrt{\alpha}}$ reduces this to

$$
-\frac{1}{\pi} \int_{\sqrt{\alpha}}^{\infty} \frac{s}{1+s^{2}} e^{-\frac{y s}{\alpha}} \cos \frac{x s}{\alpha} d s
$$

and the lower limit may be taken as zero instead of $\sqrt{\alpha}$, since

$$
\int_{0}^{\sqrt{\alpha}} \frac{s}{1+s^{2}} e^{-\frac{y s}{\alpha}} \cos \frac{x s}{\alpha} d s
$$

is infinitesimal. The second member of (11) then becomes

$$
-\frac{1}{\pi} \int_{0}^{\infty} \frac{s}{1+s^{2}} e^{-\frac{y s}{\alpha}} \cos \frac{x s}{\alpha} d s
$$

which is a finite function of the two finite ratios $\frac{x}{\alpha}$ and $\frac{y}{\alpha}$ which establishes the validity of (10). Replacing $a$ by $\frac{p}{n}$ in (10) and substituting in (3) gives

$$
G_{1}(y)=\frac{4 a^{3}}{\pi} \frac{p^{2}}{a^{2}}\left\{\frac{1}{8} \sum_{n=1}^{\infty}\left(\frac{T_{0}(n-1)+T_{0}(n+1)}{n^{2}}\right)-\frac{\pi^{2}}{12} \log \frac{z}{2 a}-\frac{1}{2} l_{1}{ }_{1}\left(\frac{z}{\dot{p}}\right)\right\}
$$

where

$$
F_{1}\left(\frac{z}{p}\right)=\frac{\partial}{\partial p} p \sum_{n=1}^{\infty} \frac{1}{n n^{2}} \int_{0}^{\infty} \frac{s e^{\mathrm{i} \frac{\mathrm{n} z}{\mathrm{p}} \mathrm{s}}}{1+s^{2}} d s
$$




$$
\text { Appendix 6.-THE INTEGRAL } \int_{0}^{\infty} f(x) J_{\mathrm{k}}\left(r_{1} x\right) J_{\mathrm{k}}\left(r_{2} x\right) d x
$$

Let $f(z)$ be an odd single-valued function of the complex variable $z$, which does not become infinite when the modulus of $z$ becomes infinite, and which assumes the real value $f^{\prime}(x)$ when $z$ assumes the real value $x$, where

$$
z=x+i y=R e^{i \theta}
$$

The real integral $\int_{0}^{\infty} f(x) J_{\mathbf{k}}\left(r_{1} x\right) J_{\mathbf{k}}\left(r_{2} x\right) d x$ (where $r_{2}>r_{1}>0$ ) is taken along the positive real axis of $z$ on which $y=0, x>0$, or simply $\theta=0$. If $-\frac{\pi}{2}<\theta \leqq \frac{\pi}{2}$ the Hankel's function $H_{1}{ }^{\mathbf{k}}(z)$ of the first kind has the asymptotic expansion (when the modulus of $z$, that is $R$, is large).

$$
H_{1}^{k}(z) \approx \frac{\sqrt{2}}{e^{i \frac{\theta}{2}} \sqrt{\pi R}}[P(z)+i Q(z)] e^{i\left(z-\frac{2 k+1}{4} \pi\right)}
$$

where $P(z)$ and $Q(z)$ have the customary meaning. Also

$$
J_{\mathbf{k}}(z)=\frac{1}{2}\left\{H_{1}^{\mathrm{k}}(z)-(-1)^{\mathbf{k}} H_{1}^{\mathrm{k}}\left(z e^{\mathrm{i} \pi}\right)\right\}
$$

Hence

$$
\begin{gathered}
\int_{0}^{\infty} f(x) J_{\mathbf{k}}\left(r_{1} x\right) J_{\mathbf{k}}\left(r_{2} x\right) d x=\frac{1}{2} \int_{0}^{\infty} f(x) J_{\mathbf{k}}\left(r_{1} x\right) H_{1}^{\mathbf{k}}\left(r_{2} x\right) d x \\
-\frac{1}{2} \int_{0}^{\infty} f(x)(-1)^{\mathbf{k}} J_{\mathbf{k}}\left(r_{1} x\right) H_{1}^{\mathbf{k}}\left(r_{2} \times e^{\mathrm{i} \pi}\right) d x
\end{gathered}
$$

In the last integral we may change the variable of integration from $x$ to $x^{1}$ where

so that it becomes

$$
x^{1}=x e^{i \pi}
$$

$$
+\frac{1}{2} \int_{0}^{-\infty} f\left(-x^{1}\right)(-1)^{\mathbf{k}} J_{\mathbf{k}}\left(-r_{1} x^{1}\right) H_{1}^{\mathbf{k}}\left(r_{2} x^{1}\right) d x^{1}
$$

or dropping the prime and placing

just

$$
f(-x)=-f(x),(-1)^{\mathbf{k}} J_{\mathbf{k}}\left(-r_{1} x\right)=J_{\mathbf{k}}\left(r_{\mathbf{1}} x\right)
$$

$$
+\frac{1}{2} \int_{-\infty}^{0} f(x) J_{\mathbf{k}}\left(r_{1} x\right) H_{1}^{\mathbf{k}}\left(r_{2} x\right) d x
$$

so that

$$
\int_{0}^{\infty} f(x) J_{\mathbf{k}}\left(r_{1} x\right) J_{\mathbf{k}}\left(r_{2} x\right) d x=\frac{1}{2 \pi i} \int_{-\infty}^{\infty} f(x) \pi i J_{\mathbf{k}}\left(r_{1} x\right) H_{1}^{\mathbf{k}}\left(r_{2} x\right) d x
$$


An infinitesimal element of length near the origin contributes a vanishing amount to this integral, so that we may imagine the path of the integral to be along the real axis from $-\infty$ to $+\infty$, passing not through the origin but going above it in an infinitesimal semicircle with the origin as center. If instead of ending at $+\infty$ the path were brought back to the starting point $-\infty$ by integrating along a semicircle with center at the origin and infinite radius, the contribution of this latter path would be zero. To show this, we make use of (1) together with the corresponding asymptotic expansion for $\mathrm{J}_{\mathbf{k}}(z)$ when the imaginary part of $z$ is positive, namely

$$
\mathrm{J}_{\mathbf{z}}(z) \approx \frac{1}{e^{\mathrm{i} \frac{\theta}{2}} \sqrt{2 \pi R}}\{P(z)-i Q(z)\} e^{-\mathrm{i}\left(\mathrm{z}-\frac{2 \mathrm{k}+1}{4} \pi\right)}
$$

Replace $z$ in (1) by $r_{2} z$, in (4) by $r_{1} z$, then $r_{1} z$ and $r_{2} z$ have the same angle $\theta$ since $r_{1}$ and $r_{2}$ are positive reals. Their moduli are $r_{1} R$ and $r_{2} R$, respectively. Hence, (1) and (4) give when $R$ is large and $0<\theta<\pi$

$$
\pi i J_{\mathbf{k}}\left(r_{1} z\right) H_{1}^{\mathbf{k}}\left(r_{2} z\right) \approx \frac{i}{\sqrt{r_{1} r_{2}}}\left(P\left(r_{2} z\right)+i Q\left(r_{2} z\right)\right)\left(P\left(r_{1} z\right)-i Q\left(r_{1} z\right)\right) \frac{e}{z}^{\mathrm{i} z\left(r_{2}-r_{1}\right)}
$$

This vanishes with infinite value of $R$ or $\bmod z$ since $z=x+i y$ and $i z=-y+i x$, and $y>0$ on the semicircle. Therefore, (3) may be written

$$
\begin{array}{r}
\int_{0}^{\infty} f(x) J_{\mathbf{k}}\left(r_{\mathbf{1}} x\right) J_{\mathbf{k}}\left(r_{2} x\right) d x=\frac{1}{2 \pi i} C \int_{\text {if } r_{2}>r_{1}>0} f(z) \pi i J_{\mathbf{k}}\left(r_{1} z\right) H_{1}^{\mathbf{k}}\left(r_{2} z\right) d z \\
\text {. }
\end{array}
$$

where the contour integral in the second member encircles positively the entire upper half of the $z$ plane. It may therefore be shrunk down about a number of infinitesimal loops, each encircling a pole of $f(z)$. If $f(z)$ has no poles in the upper half of the $z$ plane, or if the sum of their residuals vanishes, then the value of the integral is zero. In the particular case where

$$
f(z)=\frac{z}{z^{2}+\frac{n^{2}}{p^{2}}}=\frac{1}{2}\left[\frac{1}{z-\frac{i n}{p}}+\frac{1}{z+\frac{i n}{p}}\right]
$$

the simple pole at $z=\frac{i n}{p}$ gives the residue

$$
\int_{0}^{\infty} \frac{x}{x^{2}+\frac{n^{2}}{p^{2}}} J_{\mathbf{k}}\left(r_{1} x\right) J_{\mathbf{k}}\left(r_{2} x\right) d x=\frac{\pi i}{2} J_{\mathbf{k}}\left(\frac{i n r_{1}}{p}\right) H_{1}{ }^{\mathbf{k}}\left(\frac{i n r_{2}}{p}\right) \text { if } r_{2}>r_{1}
$$


This exact expression may be simplified for our present purpose because of the fact that even for the smallest value of $n$ (that is, $n=1$ ) $\frac{i n r_{1}}{p}$ and $\frac{i n r_{2}}{p}$ are very large $\left(\frac{p}{r_{1}}\right.$ is considered a first order infinitesimal $)$. In other words, the pole lies so far up on the imaginary axis of $z$ that the asymptotic expansion (5) is applicable. Placing $z=\frac{i n}{p}$ in (5) gives

$$
\begin{gathered}
\frac{\pi i}{2} J_{\mathbf{k}}\left(\frac{i n r_{1}}{p}\right) H_{1} \mathbf{k}\left(\frac{i n r_{2}}{p}\right) \approx \frac{p e^{-n\left(\frac{r_{2}-r_{1}}{p}\right)}}{2 n \sqrt{r_{1} r_{2}}}\left[P\left(\frac{i n r_{2}}{p}\right)\right. \\
\left.+i Q\left(\frac{i n r_{2}}{p}\right)\right]\left[P\left(\frac{i n r_{1}}{p}\right)-i Q\left(\frac{i n r_{1}}{p}\right)\right]
\end{gathered}
$$

This contains the small factor $\frac{p}{n}$; hence, a second order approximation will be obtained by placing $P\left(\frac{i n r_{2}}{p}\right)=P\left(\frac{i n r_{1}}{p}\right)=1$

$$
\begin{aligned}
& i Q\left(\frac{i n r_{2}}{p}\right)=\frac{p\left(k^{2}-\frac{1}{4}\right)}{2 n r_{2}} \\
& i Q\left(\frac{i n r_{1}}{p}\right)=\frac{p\left(k^{2}-\frac{1}{4}\right)}{2 n r_{1}}
\end{aligned}
$$

so that

$$
\begin{gathered}
{\left[P\left(\frac{i n r_{2}}{p}\right)+i Q\left(\frac{i n r_{2}}{p}\right)\right]\left[P\left(\frac{i n r_{1}}{p}\right)-i Q\left(\frac{i n r_{1}}{p}\right)\right]} \\
\approx 1-\frac{\left(k^{2}-\frac{1}{4}\right)}{2 n} \frac{p}{r_{2}} \frac{\left(r_{2}-r_{1}\right)}{r_{1}} \approx 1
\end{gathered}
$$

since $\frac{p}{r_{2}}$ and $\frac{r_{2}-r_{1}}{r_{1}}$ are both small. Therefore

$$
\frac{\pi i}{2} J_{\mathrm{k}}\left(\frac{i n r_{1}}{p}\right) H_{1}^{\mathrm{k}}\left(\frac{i n r_{2}}{p}\right)=\frac{p e^{-\mathrm{n}\left(\frac{\mathrm{r}_{2}-\mathrm{r}_{1}}{\mathrm{p}}\right)}}{2 n \sqrt{r_{1} r_{2}}}
$$

neglecting infinitesimals of higher order than the second in $\frac{p}{r_{2}}$ and $\frac{r_{2}-r_{1}}{r_{1}}$. Therefore the approximation to the second order inclusive for the integral in (8) is

$$
\int_{0}^{\infty} \frac{x}{x^{2}+\frac{n^{2}}{p^{2}}} J_{\mathbf{k}}\left(r_{1} x\right) J_{\mathbf{k}}\left(r_{2} x\right) d x=\frac{1}{2 \sqrt{r_{1} r_{2}}} \frac{p}{n} e^{-\mathrm{n}\left(\frac{\mathrm{r}_{2}-\mathrm{r}_{1}}{\mathrm{p}}\right)}-\text { if } r_{2}>r_{1}
$$


Another particular case of (6) is where

$$
f(z)=\frac{2 n}{p} \frac{z}{\left(z^{2}+\frac{n^{2}}{p^{2}}\right)^{2}}=\frac{1}{2 i}\left[\frac{1}{\left(z-\frac{i n}{p}\right)^{2}}-\frac{1}{\left(z+\frac{i n}{p}\right)^{2}}\right]
$$

The second order pole at $z=\frac{i n}{p}$ gives the residue

$$
\begin{gathered}
\frac{2 n}{p} \int_{0}^{\infty} \frac{x}{\left(x^{2}+\frac{n^{2}}{p^{2}}\right)^{2}} J_{\mathbf{k}}\left(r_{1} x\right) J_{\mathbf{k}}\left(r_{2} x\right) d x=\left[\frac{d}{d z} \frac{\pi}{2} J_{\mathbf{k}}\left(r_{1} z\right) H_{1}^{\mathrm{k}}\left(r_{2} z\right)\right]_{z=\frac{i n}{\mathrm{p}}} \\
\quad=\frac{1}{2 \sqrt{r_{1} r_{2}}}\left[\frac{d}{d z}\left(\frac{e^{\mathrm{iz}\left(\mathrm{r}_{1}-r_{1}\right)}}{z}\right)\right]_{z=\frac{\mathrm{in}}{\mathrm{p}}}=\frac{p}{2 \sqrt{r_{1} r_{2} n}}\left(r_{2}-r_{1}+\frac{p}{n}\right) e^{-\mathrm{n}\left(\frac{\mathrm{r}_{1}-\mathrm{r}_{1}}{\mathrm{p}}\right)}
\end{gathered}
$$

to the second order inclusive. If

Appendix 7.-TRANSFORMATIONS OF THE FUNCTION $F_{2}(z)$

$$
z=x+i y
$$

and if $x>0$, or if $x=0$ and $y \neq 2 \pi k i, k= \pm(0,1,2,3, \cdots)$ the function $F_{2}(z)$ is defined by the convergent series

$$
F_{2}(z)=\sum_{n=1}^{\infty} \frac{e^{-n s}}{n}
$$

This series defines a single-valued, periodic function of $z$ of period $2 \pi i$ in the above range of $z$.

The function $-\log \left(1-e^{-s}\right)$ is a multiple-valued function, but its principal branch is equal to the single-valued function $F_{2}(z)$. Hence, if we understand in the following that the symbol $\log \omega$ (where $\omega$ is complex) refers to the principal branch of the logarithm, we may write

$$
F_{2}(z) \equiv \sum_{n=1}^{\infty} \frac{e^{-n z}}{n}=-\log \left(1-e^{-s}\right)=-\log 2 e^{-\frac{z}{2}}\left(\frac{e^{\frac{z}{2}}-e^{-\frac{z}{2}}}{2}\right)
$$

or

$$
F_{2}(z)-\frac{1}{2} z=-\log 2 \sinh \frac{z}{2}
$$

Now

Hence

$$
2 \sinh \frac{z}{2}=z \prod_{k=1}^{\infty}\left[1+\left(\frac{z}{2 \pi k}\right)^{2}\right]
$$

$$
F_{2}(z)-\frac{z}{2}=-\log z-\sum_{k=1}^{\infty} \log \left[1+\left(\frac{z}{2 \pi k}\right)^{2}\right]
$$


If $|z|<2 \pi$ then for $k=1,2,3,4 \ldots \ldots \ldots$

$$
-\log \left[1+\left(\frac{z}{2 \pi k}\right)^{2}\right]=\sum_{n=1}^{\infty} \frac{(-1)^{n}}{n}\left(\frac{z}{2 \pi k}\right)^{2 n}
$$

and

hence

$$
\begin{gathered}
-\sum_{k=1}^{\infty} \log \left[1+\left(\begin{array}{c}
z \\
2 \pi k
\end{array}\right)^{2}\right]=\sum_{k=1}^{\infty} \sum_{n=1}^{\infty} \frac{(-1)^{n}}{n}\left(\frac{z}{2 \pi k}\right)^{2 n} \\
=\sum_{n=1}^{\infty} \frac{(-1)^{n}}{n}\left(\frac{z}{2 \pi}\right)^{2 n} \sum_{k=1}^{\infty} \frac{1}{k^{2 n}}
\end{gathered}
$$

where

$$
\begin{gathered}
F_{2}\left(\frac{z}{p}\right)-\frac{1}{2} \frac{z}{p}=-\log \frac{z}{p}+\sum_{n=1}^{\infty} b_{\mathrm{n}}\left(\frac{z}{2 \pi p}\right)^{2 n} \ldots \text { if }|z|<2 \pi p \\
\text { or if }|z|=2 \pi p \text { ad } x \neq 0
\end{gathered}
$$

$$
b_{\mathrm{n}}=\frac{(-1)^{\mathrm{n}}}{n} \sum_{k=1}^{\infty} \frac{1}{k^{2 \mathrm{n}}}=\frac{(-1)^{\mathrm{n}}}{2 n} \frac{(2 \pi)^{2 \mathrm{n}}}{\underline{2 n}} B_{\mathrm{n}}
$$

where the $B_{\mathrm{n}} s$ are Bernoulli's numbers

$$
\begin{aligned}
& b_{1}=-\frac{\pi^{2}}{6}=-1.644934 \\
& b_{2}=+\frac{1}{2}\left(\frac{\pi^{4}}{90}\right)=+\frac{1}{2}(1.082323) \\
& b_{3}=-\frac{1}{3}\left(\frac{\pi^{6}}{945}\right)=-\frac{1}{3}(1.017343) \\
& b_{4}=+\frac{1}{4}\left(\frac{\pi^{8}}{9450}\right)=+\frac{1}{4}(1.004077) \\
& b_{5} \quad=-\frac{1}{5}(1.000995)
\end{aligned}
$$

so that $b_{\mathrm{n}}=\frac{(-1)^{\mathrm{n}}}{n}$ very approximately if $n$ is large.

The expansion (8) will be convenient to use in case no two points of the section $S$ of the wire are separated by a distance greater than $2 \pi p$, the pitch of the helix. Such would be the case for a square or circular wire, or a rectangular wire whose diagonal does not exceed the pitch of the helix. If the diagonal $\rho$ exceeds the pitch $2 \pi p$, but is less than twice this value, one might use an expansion analogous to (8) by taking out the term $\log \left[1+\left(\frac{z}{2 \pi p}\right)^{2}\right]$. The remaining series would then converge for the range $|z|<4 \pi p$. 
Appendix 8.-TRANSFORMATIONS OF $F_{1}(z)$

$$
F_{1}(z)=\sum_{n=1}^{\infty} \frac{1}{n^{2}} \int_{0}^{\infty} e^{\mathrm{inzs}}\left(\frac{s-i n z s^{2}}{1+s^{2}}\right) d s=\frac{\pi^{2}}{6}+\sum_{n=1}^{\infty} \frac{1}{n^{2}} \int_{0}^{\infty} e^{\mathrm{inzs}}\left(\frac{s+i n z}{1+s^{2}}\right) d s
$$

Consider the integral

$$
\int_{0}^{\infty} e^{\mathrm{inzs}}\left(\frac{s+i n z}{1+s^{2}}\right) d s
$$

Instead of the positive real axis of $s$, the path of this integral may be taken as any path in the complex $s$ plane, which starts at the origin and goes to infinity in any direction in which the integrand vanishes with infinite value of $s$, provided also that this path may be deformed into the positive real axis without passing through either of the two poles of the integrand, which are at $s= \pm i$. Let the path be a straight line radiating from the origin, whose angle is $\alpha$; that is, one obtained by rotating the positive real axis through an angle $\alpha$. Let

$$
z=\rho e^{\mathrm{i} \theta}=x+i y \text { where } x>0 ; y>0, o<\theta<\frac{\pi}{2}, s=R e^{\mathrm{i} \alpha}, d s=e^{\mathrm{i} \alpha} d R
$$

on the new path, so that

$$
\begin{gathered}
\int_{0}^{\infty} e^{\mathrm{inzs}}\left(\frac{s+i n z}{1+s^{2}}\right) d s \\
=\int_{0}^{\infty} \frac{R+i n \rho e^{\mathrm{i}(\theta-\alpha)}}{R^{2}+e^{-2 \mathrm{i} \alpha}} e^{-\mathrm{n} \rho \mathrm{R} \sin (\theta+\alpha)+\mathrm{in} \rho \mathrm{R} \cos (\theta+\alpha)} d R
\end{gathered}
$$

The variable of integration is here the modulus $R$ which is real and positive. It is evident that the integral on the right will converge, and that over the circular are at infinity vanish, provided that $0 \leqq \alpha+\theta \leqq \pi$; that is, $-\theta \leqq \alpha \leqq \pi-\theta$ with the exception of the value $\alpha=\frac{\pi}{2}$ in which case the pole $s=i$ would lie upon the line. The two cases which will be useful are the two limits of the range of $\alpha$. In the first case take $\alpha=-\theta$. This negative rotation of the axis encounters no poles; hence this gives

$$
\begin{aligned}
\int_{0}^{\infty} e^{\mathrm{inzz}} \frac{s+i n z}{1+s^{2}} d s & =\int_{0}^{\infty}\left(\frac{R+i n \rho e^{2 \mathrm{i} \theta}}{R^{2}+e^{2 i \theta}}\right) e^{\mathrm{in} \rho \mathrm{R}} d R \\
& =\int_{0}^{\infty}\left(\frac{t+i n \rho^{2} e^{2 \mathrm{i} \theta}}{t^{2}+\rho^{2} e^{2 \mathrm{i} \theta}}\right) e^{\mathrm{int}} d t \\
& =\int_{0}^{\infty}\left(\frac{t+i n z^{2}}{t^{2}+z^{2}}\right) e^{i \mathrm{in}} d t
\end{aligned}
$$


A second expression for the integral on the left may be obtained by taking $\alpha=\pi-\theta$. In thus rotating the positive real axis through the positive angle $\pi-\theta$, the simple pole $s=i$ is passed and leares a residual circuit integral about a loop inclosing the pole whose value is $\pi i(1+n z) e^{-\mathrm{nz}}$. This gives $\Omega$ second expression

$$
\int_{0}^{\infty} e^{\operatorname{lnz\theta }}\left(\frac{s+i n z}{1+s^{2}}\right) d s=\pi i(1+n z) e^{-n z}+\int^{\infty}\left(\frac{t-i n z^{2}}{t^{2}+z^{2}}\right) e^{-\mathrm{nat}} d t
$$

Adding these two expressions (3) and (4) for the integral on the left, and dividing by two, gives

$$
\int_{0}^{\infty} e^{\operatorname{lnz} \frac{s+i n z}{1+s^{2}}} d s=i \frac{\pi}{2}(1+n z) e^{-\mathrm{nz}}+\int_{0}^{\infty} \frac{t \cos n t-n z^{2} \sin n t}{t^{2}+z^{2}} d t
$$

Hence, summing with respect to $n$ gives

$$
\begin{aligned}
F_{1}(z) & =\frac{\pi^{2}}{6}+\frac{i \pi}{2}\left[z \sum_{n=1}^{\infty} \frac{e^{-\mathrm{nz}}}{n}+\sum_{n=1}^{\infty} \frac{e^{-\mathrm{n}}}{n^{2}}\right] \\
& +\int_{0}^{\infty} \frac{d t}{z^{2}+t^{2}}\left[t \sum_{n=1}^{\infty} \frac{\cos n t}{n^{2}}-z^{2} \sum_{n=1}^{\infty} \frac{\sin n t}{n}\right]
\end{aligned}
$$

or

$$
-\frac{2}{\pi} F_{1}(z)+i\left[z F_{2}(z)+F_{3}(z)\right]=-\frac{\pi}{2}\left\{\frac{2}{3}+\int_{0}^{\infty} \frac{d t}{z^{2}+t^{2}}\left[t \psi(t)+z^{2} \psi^{\prime}(t)\right]\right\}
$$

where

$$
\begin{aligned}
& \psi(t)=\frac{4}{\pi^{2}} \sum_{n=1}^{\infty} \frac{\cos n t}{n^{2}}=\frac{2}{3}-2 \frac{t}{\pi}+\frac{t^{2}}{\pi^{2}} \text { if } o \leqq t \leqq 2 \pi \\
& \psi^{\prime}(t)=-\frac{4}{\pi^{2}} \sum_{n=1}^{\infty} \frac{\sin n t}{n}=\frac{2}{\pi}\left(\frac{t}{\pi}-1\right) \ldots \text { if } o \leqq t \leqq 2 \pi
\end{aligned}
$$

while $\psi(t+2 \pi n)=\psi(t)$ and $\psi^{\prime}(t+2 \pi n)=\psi^{\prime}(t)$.

The functions $F_{2}$ and $F_{3}$ are the series defined by equation (78) in the text.

The second member of (7) may be further simplified by taking account of the periodicity of $\psi(t)$ and writing the integral

$$
\int_{0}^{\infty}() d t=\int_{0}^{2 \pi}() d t+\int_{2 \pi}^{4 \pi}(\quad) d t \ldots \int_{2 \pi n}^{2 \pi(\mathrm{n}+1)}(\quad) d t+\ldots
$$


This gives

$$
\begin{aligned}
& -\frac{2}{\pi} F_{1}(z)+i z F_{2}(z)+i F_{3}(z)=-\pi\left\{\frac{1}{3}\right. \\
& \left.+2 \sum_{n=0}^{\infty} \int_{0}^{1} d t \frac{\left(t^{2}-t+\frac{1}{6}\right)(t+n)+\left(\frac{z}{2 \pi}\right)^{2}(2 t-1)}{(t+n)^{2}+\left(\frac{z}{2 \pi}\right)^{2}}\right\} \\
& =-\pi\left\{\frac{1}{3}+2 \sum_{n=0}^{\infty} \int_{0}^{1} d t(t-(n+1)+[n(n+1)\right. \\
& \left.+\left(\frac{z}{2 \pi}\right)^{2}+\frac{1}{6}\right] \frac{t+n}{\left.\left.(t+n)^{2}+\left(\frac{z}{2 \pi}\right)^{2}\right)\right\}} \\
& =+\pi\left\{\frac{2}{3}+2\left[\left(\frac{z}{2 \pi}\right)^{2}+\frac{1}{6}\right] \log \left(\frac{z}{2 \pi}\right)+\sum_{n=1}^{\infty} u_{n}+\sum_{n=1}^{\infty} v_{0}(z)\right\}
\end{aligned}
$$

where

$$
\begin{gathered}
u_{n}=2 n+1-\left[n(n+1)+\frac{1}{6}\right] 2 \log \left(1+\frac{1}{n}\right) \\
v_{n}(z)=n(n+1) \log \frac{1+\left(\frac{z}{2 \pi n}\right)^{2}}{1+\left(\frac{z}{2 \pi(n+1)}\right)^{2}}-2\left(\frac{z}{2 \pi}\right)^{2} \log \left(1+\frac{1}{n}\right)
\end{gathered}
$$

To sum the (numerical) $u_{n-}$ series (9), we may expand the term $\log \left(1+\frac{1}{n}\right)$ in ascending powers of $\frac{1}{n}$. This gives

$$
u_{\mathrm{n}}=\sum_{k=3}^{\infty} \frac{(-1)^{\mathbf{k}}}{n^{\mathbf{k}}}\left[\frac{1}{3 k}-\frac{2}{(k+1)(k+2)}\right]
$$

which leads to the expression of (9) as a double series

or

$$
\sum_{n=1}^{\infty} u_{\mathbf{n}}=\sum_{n=1}^{\infty} \sum_{k=3}^{\infty} \frac{(-1)^{\mathbf{k}}}{n^{\mathbf{k}}}\left[\frac{1}{3 k}-\frac{2}{(k+1)(k+2)}\right]
$$

$$
\sum_{n=1}^{\infty} u_{n}=\sum_{k=3}^{\infty}(-1)^{\mathrm{k}}\left[\frac{1}{3 k}-\frac{2}{(k+1)(k+2)}\right] \sum_{n=1}^{\infty} \frac{1}{n^{k}}
$$


To increase the rapidity of convergence of this series, we may note that for large values of $k$ the sum $\sum_{n=1}^{\infty} \frac{1}{n^{\mathbf{k}}}$ is very nearly equal to unity so that the $k^{\text {th }}$ term approaches $(-1)^{\mathrm{k}}\left[\frac{1}{3 k}-\frac{2}{(k+1)(k+2)}\right]$ which corresponds to the development

$$
\begin{gathered}
-\frac{1}{3} \log (1+x)-\frac{2}{x^{2}}[(1+x) \log (1+x)-x]+1 \\
=\sum_{k=3}^{\infty}(-1)^{\mathbf{k}}\left[\frac{1}{3 k}-\frac{2}{(k+1)(k+2)}\right] x^{\mathbf{k}}
\end{gathered}
$$

For $x=1$ this derelopment (13) gives

$$
0=3-\frac{13}{3} \log 2-\sum_{k=3}^{\infty}(-1)^{\mathrm{k}}\left[\frac{1}{3 k}-\frac{2}{(k+1)(k+2)}\right]
$$

Adding this identically zero quantity to (12) gires

$$
\sum_{n=1}^{\infty} u_{\mathrm{n}}=3-\frac{13}{3} \log 2+\sum_{k=3}^{\infty}(-1)^{\mathrm{k}}\left[\frac{1}{3 k}-\frac{2}{(k+1)(k+2)}\right]\left[\sum_{n=1}^{\infty} \frac{1}{n^{\mathrm{k}}}-1\right]
$$

The sum of the first 15 terms of the series on the right is -0.000345 , and this differs from the series by less than 0.000001 . Hence

$$
\sum_{n=1}^{\infty} u_{n}=3-\frac{13}{3} \log 2-0.000345=-0.00400
$$

To simplify the $v_{\mathrm{n}}(z)$ series of (8), we proceed in a similar manner to expand the logarithms in expression (10) for $v_{n}(z)$. This gives if $|z|<2 \pi$

$$
v_{\mathrm{n}}(z)=\sum_{k=1}^{\infty} \frac{(-1)^{\mathrm{k}+1}}{7}\left(\frac{z}{2 \pi}\right)^{2 \mathrm{k}} C_{n \mathrm{~s}}
$$

where

$$
C_{n_{1}}=-\frac{1}{n(n+1)}+2\left[\frac{1}{2 n^{2}}-\frac{1}{3 n^{3}}+\frac{1}{4 n^{4}}-\frac{1}{5 n^{5}}+\cdots\right]
$$

and if $k>1$

$$
C_{\mathrm{nk}}=\frac{1}{n^{2 \mathrm{~L}-2}}-\frac{1}{(n+1)^{2 \mathrm{k}-2}}+\frac{1}{n^{2 \mathrm{k}-1}}-\frac{1}{(n+1)^{2 \mathrm{k}-1}}
$$


Using these expressions leads to the following double series for the $v_{\mathbf{n}}(z)$ series in (8):

$$
\sum_{n=1}^{\infty} v_{\mathrm{n}}(z)=\sum_{n=1}^{\infty} \sum_{k=1}^{\infty} \frac{(-1)^{\mathrm{k}+1}}{k}\left(\frac{z}{2 \pi}\right)^{2 \mathrm{k}} C_{\mathrm{nk}}
$$

or

where

$$
\sum_{n=1}^{\infty} v_{\mathrm{n}}(z)=c_{1}\left(\frac{z}{2 \pi}\right)^{2}-2 \sum_{n=2}^{\infty} c_{\mathrm{n}}\left(\frac{z}{2 \pi}\right)^{2 \mathrm{n}}
$$

$$
\begin{aligned}
c_{1} & =\sum_{k=1}^{\infty}\left\{-\frac{1}{k(k+1)}+2\left[\frac{1}{2 k^{2}}-\frac{1}{3 k^{3}}+\frac{1}{4 k^{4}}-\frac{1}{5 k^{5}}+\ldots\right]\right\} \\
& =1-2 \log 2+2\left[\frac{1}{2}\left(\sum_{k=1}^{\infty} \frac{1}{k^{2}}-1\right)-\frac{1}{3}\left(\sum_{k=1}^{\infty} \frac{1}{k^{3}}-1\right)+\frac{1}{4}\left(\sum_{k=1}^{\infty} \frac{1}{k^{4}}-1\right)-\right. \\
& =1-2 \log 2+0.54072=0.15442
\end{aligned}
$$

and

$$
c_{\mathrm{n}}=\frac{(-1)^{\mathrm{n}}}{n} \sum_{k=1}^{\infty} \frac{1}{k^{2 \mathrm{n}-1}} \text { if } n>1
$$

Making use of (16) and (19) in (8) gives

$$
\begin{aligned}
& 2\left[-\frac{2}{\pi} F_{1}\left(\frac{z}{p}\right)+i \frac{z}{p} F_{2}\left(\frac{z}{p}\right)+i F_{3}\left(\frac{z}{p}\right)\right]-\pi\left[\frac{2}{3}+4\left(\frac{z}{2 \pi p}\right)^{2}\right] \log \left(\frac{z}{2 \pi p}\right) \\
& +4 \pi\left(\frac{z}{2 \pi p}\right) \log \frac{4}{\pi}=2 \pi\left\{0.66267+0.63754\left(\frac{z}{2 \pi p}\right)^{2}-2 \sum_{n=2}^{\infty} c_{\mathrm{n}}\left(\frac{z}{2 \pi p}\right)^{2 \mathrm{n}}\right\}(22)
\end{aligned}
$$

Appendix 9.-ANALYTICAL METHOD OF EVALUATING THE INTEGRAL $B_{0}$

$$
B_{0}=\int_{0}^{\eta \equiv \frac{\sqrt{1-\mathrm{k}^{2}}}{\mathrm{k}}} d \eta_{2} \int_{0}^{\eta_{3}} d \eta_{1} \frac{K\left(\frac{1}{\sqrt{1+\eta_{1}^{2}}}\right)}{\sqrt{1+\eta_{1}{ }^{2}}}=\int_{0}^{\eta} \frac{\eta-\eta_{1}}{\sqrt{1+\eta_{1}{ }^{2}}} F\left(\frac{1}{\sqrt{1+\eta^{2}}}\right) d \eta_{1}
$$

(Interchanging the order of integration and performing the integration with respect to $\eta_{2}$.) If we now let $k^{1}=\frac{1}{\sqrt{1+\eta_{1}^{2}}}$ this becomes

$$
B_{0}=\frac{\sqrt{1-k^{2}}}{k} \int_{k}^{1} \frac{K\left(k^{1}\right) d k^{1}}{k^{1} \sqrt{1-k^{12}}}-\int_{k}^{1} \frac{K\left(k^{1}\right) d k^{1}}{7^{1^{2}}}
$$

Now

$$
-\frac{K\left(h^{1 \cdot 1}\right)}{k^{1^{2}}}=\frac{d}{d k^{1}}\left(\frac{E\left(k^{1}\right)}{k^{1}}\right)
$$




\section{Hence}

$$
B_{0}=1-\frac{E(k)}{k}+\frac{\sqrt{1-k^{2}}}{k} \int_{\mathrm{k}}^{1} \frac{K\left(k^{1}\right) d k^{1}}{k^{1} \sqrt{1-k^{12}}}
$$

If we next let $k^{1}=\frac{2 \lambda^{3}}{1+\lambda}$ and note that by Landen's transformation $K\left(\frac{2 \lambda^{1}}{1+\lambda}\right)=(1+\lambda) K(\lambda)$ then $\frac{d k^{1}}{k^{1} \sqrt{1-k^{1^{2}}}}=\frac{d \lambda}{2 \lambda}$ and the preceding integral becomes

$$
\int_{0}^{1} \frac{K\left(k^{1}\right) d k^{1}}{k^{1} \sqrt{1-k^{12}}}=\frac{1}{2} \int_{\mathrm{k}_{1}}^{1}\left(1+\frac{1}{\lambda}\right) K(\lambda) d \lambda
$$

where

$$
k_{1}=\frac{1-\sqrt{1-k^{2}}}{1+\sqrt{1-k^{2}}} \text { so that } k=\frac{2 k_{1}^{\frac{1}{2}}}{1+k_{1}} \text { and } \sqrt{1-k^{2}}=\frac{1-k_{1}}{1+k_{1}}
$$

Now

$$
\begin{aligned}
& \int_{\mathrm{k}}^{1} \frac{K\left(k^{1}\right) d k^{1}}{k^{1} \sqrt{1-k^{1^{2}}}}=\frac{1}{2} \int_{\mathrm{k}_{1}}^{1}\left(1+\frac{1}{\lambda}\right) K(\lambda) d \lambda \\
& =\frac{\pi}{4} \int_{\mathrm{k}_{1}}^{1} d \lambda\left(1+\frac{1}{\lambda}\right)\left(1+\frac{1}{\pi} \sum_{n=1}^{\infty}\left[\frac{\Gamma\left(n+\frac{1}{2}\right)}{\Gamma(n+1)}\right]^{2} \lambda^{2 \mathrm{n}}\right) \\
& =\frac{\pi}{4}\left\{\log \frac{1}{k_{1}}+1-k_{1}+\frac{1}{\pi} \sum_{n=1}^{\infty}\left[\frac{\Gamma\left(n+\frac{1}{2}\right)}{\Gamma(n+1)}\right]^{2}\left(\frac{1}{2 n+1}+\frac{1}{2 n}\right)\right. \\
& \left.-\frac{1}{\pi} \sum_{n=1}^{\infty}\left[\frac{\Gamma\left(n+\frac{1}{2}\right)}{\Gamma(n+1)}\right]^{2}\left(\frac{k_{1}{ }^{2 \mathrm{n}+1}}{2 n+1}+\frac{k_{1}{ }^{2 \mathrm{n}}}{2 n}\right)\right\} \\
& =\frac{\pi}{4}\left\{\log \frac{4}{k_{1}}-k_{1}-\sum_{n=1}^{\infty}\left[\frac{1.3 .5 \ldots \ldots(2 n-1)}{2.4 .6 . . .-2 n}\right]^{2}\left(\frac{k_{1}{ }^{2 n+1}}{2 n+1}+\frac{k_{1}{ }^{2 n}}{2 n}\right)\right\}
\end{aligned}
$$

Hence

$$
\begin{aligned}
& B_{0} \equiv \int_{0}^{\frac{\sqrt{1-\mathrm{k}_{2}}}{\mathrm{k}}} d \eta_{2} \int_{0}^{\eta_{2}} d \eta_{1} \frac{K\left(\frac{1}{\sqrt{1+\eta_{1}{ }^{2}}}\right)}{\sqrt{1+\eta_{1}{ }^{2}}}= \\
& =1-\frac{E(k)}{k}+\frac{\pi\left(1-k_{1}\right)}{8 \sqrt{k_{1}}}\left\{\log \frac{4}{k_{1}}\right. \\
& \left.-k_{1}-\sum_{n=1}^{\infty}\left[\frac{1.3 .5 \ldots \ldots(2 n-1)}{2.4 .6 \ldots .2 n}\right]^{2}\left(\frac{k_{1}{ }^{2 n+1}}{2 n+1}+\frac{k_{1}{ }^{2 n}}{2 n}\right)\right\}
\end{aligned}
$$

where

$$
k_{1}=\frac{1-\sqrt{1-k^{2}}}{1+\sqrt{1-k^{2}}}
$$


The constant $B_{0}$ may be accurately computed by this formula. It represents the contribution of the axial or $y$ component of current to the inductance of a continuous cylindrical current sheet consisting of tape windings without insulation space between them, for the inductance $\Delta L$, due to a $y$ component of current only in the limiting case of a cylindrical current sheet, is

$$
\begin{aligned}
\Delta L & =\frac{1}{4 \pi^{2}} \int_{0}^{l} d y \int_{0}^{l} d y^{1} \int_{0}^{2 \pi} d \theta \int_{0}^{2 \pi} d \theta^{1} \frac{1}{\sqrt{\left(y-y^{1}\right)^{2}+4 a^{2} \sin ^{2}\left(\frac{\theta^{1}-\theta}{2}\right)}} \\
& =\frac{1}{\pi^{2}} \int_{0}^{l} d y \int_{0}^{l} d y^{1} \int_{0}^{2 \pi} d \theta \int_{0}^{\frac{\pi}{2}} d \phi \frac{1}{\sqrt{\left(y-y^{1}\right)^{2}+4 a^{2} \sin ^{2} \phi}} \\
& =\frac{2}{\pi} \int_{0}^{l} d y \int_{0}^{l} d y^{1} \int_{0}^{\frac{\pi}{2}} \frac{d \phi}{\sqrt{\left(y-y^{1}\right)^{2}+4 a^{2} \sin ^{2} \phi}} \\
& =\frac{4}{\pi} \int_{0}^{l} d y \int_{0}^{\mathrm{y}} d y^{1} \int_{0}^{\frac{\pi}{2}} \frac{d \phi}{\sqrt{\left(y-y^{1}\right)^{2}+4 a^{2} \sin ^{2} \phi}} \\
& =\frac{8 a}{\pi} \int_{0}^{\eta=\frac{l}{2 a}} d \eta_{2} \int_{0}^{\eta_{2}} d \eta_{1}^{\frac{\pi}{2}} \frac{d \phi}{\sqrt{1-\frac{1}{1+\eta_{1}{ }^{2}} \sin ^{2} \phi}} \\
& =\frac{8 a}{\pi} \int_{0}^{\eta} d \eta_{2} \int_{0}^{\eta_{2}} a \eta_{1} \frac{\pi\left(\frac{1}{\sqrt{1+\eta_{1}{ }^{2}}}\right)}{\sqrt{1+\eta_{1}{ }^{2}}}=\frac{8 a}{\pi} B_{0}
\end{aligned}
$$

Appendix 10.-THE MAGNETIC FIELD OF AN IN FINITELY LONG HELICAL CURRENT FILAMENT

Imagine a helical filament having $N$ turns to the right of the plane $y=0$, and $N$ to the left, whose total length is $2 l$ where $l=2 \pi p N$. This helical filament winds around the $y$ axis and lies on the cylinder $r=r_{1}$. The points where it cuts the plane $z=0$ are $\begin{aligned} & x=r_{1} \\ & y=y_{1}\end{aligned}$ or $\begin{aligned} & x=r_{1} \\ & y=y_{1}\end{aligned}$ $\pm 2 \pi k$ where $0<y_{1}<2 \pi p$ where $k=0,1,2,3,4 \ldots \ldots \ldots$ The three quantities $r_{1}, y_{1}$, and $p$ characterize a helical filament extending indefinitely in both directions along the $y$ direction. Its field is the limiting field approached by that of the helix of length, $2 l$ when $l$ increases indefinitely in both directions. The component of magnetic field will be found to approach finite limits, although the vector potential from which it is derived, and which is a Newtonian integral, becomes logarithmically infinite. However, it is easy to find a finite and continuous logarithmic vector potential which serves 
to define the field in this case. 'The limiting field may be found by first computing the rector potential due to the finite helix extending from $y=-l$ to $y=+l$ at a point $P_{2}\left\{\begin{array}{l}x=r_{2} \\ y=y_{2} \\ z=0\end{array}\right\}$ where $-l<y_{2}<l$.

It is evident from symmetry that when $l$ becomes infinite the potential will not be a function of $\theta_{2}$ but of $r_{2}$ and $y_{2}$ only, and will be a periodic function of $y_{2}$ for a given value of $r_{2}$. These components are by (18)

$$
\begin{aligned}
& A_{\theta}\left(r_{2}, y_{2}, 0\right)=r_{1} \int_{-2 \pi \mathrm{N}}^{2 \pi \mathrm{N}} \frac{\cos \theta_{1} d \theta_{1}}{\sqrt{R^{2}+\left(y_{2}-y_{1}-p \theta_{1}\right)^{2}}} \\
& A_{\mathrm{r}}\left(r_{2}, y_{2}, 0\right)=-r_{1} \int_{-2 \pi \mathrm{N}}^{2 \pi \mathrm{N}} \frac{\sin \theta_{1} d \theta_{1}}{\sqrt{R^{2}+\left(y_{2}-y_{1}-p \theta_{1}\right)^{2}}} \\
& A_{\mathrm{y}}\left(r_{2}, y_{2}, 0\right)=p \int_{-2 \pi \mathrm{N}}^{2 \pi \mathrm{N}} \frac{d \theta_{1}}{\sqrt{R^{2}+\left(y_{2}-y_{1}-p \theta_{1}\right)^{2}}}
\end{aligned}
$$

where there is a unit current in the helical filament which is wound on the cylinder of radius $r_{1}$ and has a pitch $2 \pi p$, and which goes through $x=r_{1}$ the point $y=y_{1} \quad$ where $0 \leqq y_{1} \leqq 2 \pi p$. and where

$$
z=0 \text { or } \theta=0
$$

$$
R^{2}=r_{1}^{2}+r_{2}^{2}-2 r_{1} r_{2} \cos \theta_{1}
$$

The magnetic field at $P_{2}\left(r_{2}, y_{2}, 0\right)$ will be independent of $\theta_{2}$; that is, functions of the two cylindrical coordinates $r_{2}$ and $y_{2}$ only, as will the vector potential. Hence

$$
\begin{aligned}
& H_{\mathrm{r}}\left(r_{2}, y_{2}, 0\right)=-\frac{\partial}{\partial y_{2}} A_{\theta}\left(r_{2}, y_{2}, 0\right) \\
& H_{\theta}\left(r_{2}, y_{2}, 0\right)=\frac{\partial}{\partial y_{2}} A_{\mathrm{r}}\left(r_{2}, y_{2}, 0\right)-\frac{\partial}{\partial r_{2}} A_{\mathrm{y}}\left(r_{2}, y_{2}, 0\right) \\
& H_{\mathrm{y}}\left(r_{2}, y_{2}, 0\right)=\frac{1}{r_{2}} \frac{\partial}{\partial r_{2}}\left(r_{2} A_{\theta}\left(r_{2}, y_{2}, 0\right)\right)
\end{aligned}
$$

If we make use of Neumann's theorem in the form

$$
\begin{array}{cc}
\frac{1}{\sqrt{R^{2}+\left(y_{2}-y_{1}-p \theta_{1}\right)^{2}}}=2 \int_{0}^{\infty} d s e^{-\mid y_{1}-y_{1}-p \theta_{1} / s} \sum_{n=0}^{\infty} \epsilon_{\mathrm{n}} J_{\mathrm{n}}\left(r_{1} s\right) J_{\mathrm{n}}\left(r_{2} s\right) \cos n \theta_{1} \\
\text { where } \epsilon_{0}=\frac{1}{2} \\
\epsilon_{\mathrm{n}}=1 \text { if } n>0
\end{array}
$$


we find

$$
\left\{\begin{aligned}
A_{\theta}\left(r_{2}, y_{2}, 0\right) & =r_{1} \sum_{n=0}^{\infty} \epsilon_{\mathrm{n}} \int_{0}^{\infty} d s\left[J_{\mathrm{n}+1}\left(r_{1} s\right) J_{\mathrm{n}+1}\left(r_{2} s\right)\right. \\
& \left.+J_{\mathrm{n}-1}\left(r_{1} s\right) J_{\mathrm{n}-1}\left(r_{2} s\right)\right] \int_{-2 \pi \mathrm{N}}^{2 \pi \mathrm{N}} d \theta_{1} \cos n \theta_{1} e^{-\left|\mathrm{y}_{2}-\mathrm{y}_{1}-\mathrm{p} \theta_{1}\right| \mathrm{s}} \\
A_{\mathrm{r}}\left(r_{2}, y_{2}, 0\right) & =r_{1} \sum_{n=1}^{\infty} \int_{0}^{\infty} d s\left[J_{\mathrm{n}+1}\left(r_{1} s\right) J_{\mathrm{n}+1}\left(r_{2} s\right)\right. \\
& \left.-J_{\mathrm{n}-1}\left(r_{1} s\right) J_{\mathrm{n}-1}\left(r_{2} s\right)\right] \int_{-2 \pi \mathrm{N}}^{2 \pi \mathrm{N}} d \theta_{1} \sin n \theta_{1} e_{2}-\left|\mathrm{y}_{1}-\mathrm{y}-\mathrm{p} \theta_{1}\right| \mathrm{s} \\
A_{\mathrm{y}}\left(r_{2}, y_{2}, 0\right) & =p \sum_{n=0}^{\infty} \epsilon_{\mathrm{n}} \int_{0}^{\infty} d s J_{\mathrm{n}}\left(r_{1} s\right) J_{\mathrm{n}}\left(r_{2} s\right) \\
& \left.+J_{\mathrm{n}}\left(r_{1} s\right) J_{\mathrm{n}}\left(r_{2} s\right)\right] \int_{-2 \pi \mathrm{N}}^{2 \pi \mathrm{N}} d \theta_{1} \cos n \theta_{1} e^{-\left|\mathrm{y}_{2}-\mathrm{y}_{1}-\mathrm{p} \theta_{1}\right| \mathrm{s}}
\end{aligned}\right.
$$

now

$$
\begin{gathered}
\int_{-2 \pi \mathrm{N}}^{2 \pi \mathrm{N}} d \theta_{1} \cos n \theta_{1} e^{-\left|\mathrm{y}_{2}-\mathrm{y}_{1}-\mathrm{p} \theta_{1}\right| \mathrm{s}}=\frac{2 p s \cos \frac{n}{p}\left(y_{2}-y_{1}\right)}{p^{2} s^{2}+n^{2}} \\
-\frac{p s\left[e^{-\left(l+\mathrm{y}_{2}-\mathrm{y}_{1}\right) \mathrm{s}}+e^{-\left(l-\mathrm{y}_{2}+\mathrm{y}_{1}\right) \mathrm{s}}\right]}{p^{2} s^{2}+n^{2}}
\end{gathered}
$$

and

$$
\begin{gathered}
\int_{-2 \pi \mathrm{N}}^{2 \pi \mathrm{N}} d \theta_{1} \sin n \theta_{1} e^{-\left|\mathrm{y}_{2}-\mathrm{y}_{1}-\mathrm{p} \theta_{1}\right| \mathrm{s}}=\frac{2 p s \sin \frac{n}{p}\left(y_{2}-y_{1}\right)}{p^{2} s^{2}+n^{2}} \\
+\frac{n\left[e^{-\left(l+\mathrm{y}_{2}-\mathrm{y}_{1}\right) \mathrm{s}}-e^{-\left(l-\mathrm{y}_{2}+\mathrm{y}_{1}\right) \mathrm{s}}\right]}{p^{2} s^{2}+n^{2}} .
\end{gathered}
$$

Consider first the axial component $A_{y}\left(r_{2}, y_{2}, o\right)$ which becomes

$$
\begin{gathered}
A_{\mathrm{y}}\left(r_{2}, y_{2}, 0\right)=4 \sum_{n=0}^{\infty} \epsilon_{\mathrm{n}}^{\infty} d s \frac{s}{s^{2}+\frac{n^{2}}{p^{2}}} J_{\mathrm{n}}\left(r_{1} s\right) J_{\mathrm{n}}\left(r_{2} s\right)\left\{\cos \frac{n}{p}\left(y_{2}-y_{1}\right)\right. \\
\left.-\frac{e^{-\left(l+\mathrm{y}_{2}-\mathrm{y}_{1}\right) \mathrm{s}}+e^{-\left(l-\mathrm{y}_{2}+\mathrm{y}_{1}\right) s}}{2}\right\}
\end{gathered}
$$


or

$$
\begin{aligned}
& A_{\mathbf{y}}\left(r_{2}, y_{2}, o\right)=2 \int_{0}^{\infty} d s \frac{J_{0}\left(r_{1} s\right) J_{0}\left(r_{2} s\right)}{s}\left\{1-\frac{e^{-\left(l+\mathrm{y}_{2}-\mathrm{y}_{1}\right) \mathrm{s}}+e^{-\left(l-\mathrm{y}_{1}+\mathrm{y}_{1}\right) s}}{2}\right\} \\
&+4 \sum_{n=1}^{\infty} \cos n\left(\frac{\left.y_{2}-y_{1}\right)}{p} \int_{0}^{\infty} d s \frac{s J_{\mathrm{n}}\left(r_{1} s\right) J_{\mathrm{n}}\left(r_{2} s\right)}{s^{2}+\frac{n^{2}}{p^{2}}}\right. \\
&-2 \sum_{n=1}^{\infty} \int_{0}^{\infty} d s \cdot \frac{s J_{\mathrm{n}}\left(r_{1} s\right) J_{\mathrm{n}}\left(r_{2} s\right)}{s^{2}+\frac{n^{2}}{p^{2}}}\left\{e^{-\left(l+\mathrm{y}_{2}-\mathrm{y}_{1}\right) s}+e^{-\left(l-\mathrm{y}_{2}+\mathrm{y}_{1}\right) s}\right\}
\end{aligned}
$$

Now when $l \rightarrow \infty$ the last sum vanishes if $-l<y_{2}-y_{1}<+l$. The second sum approaches a finite limit, but the first integral becomes infinite logarithmically, since

$$
\int_{0}^{\infty} d s \frac{J_{0}\left(r_{1} s\right) J_{0}\left(r_{2} s\right)}{s}
$$

diverges at the lower limit. However, this term contributes only a finite amount (in the limit) to the magnetic field component $H_{\theta}\left(r_{2}\right.$, $y_{2}, o$ ) by the second of equations (3) which is

$$
-\frac{\partial}{\partial r_{2}} 2 \int_{0}^{\infty} d s \frac{\left.J_{0}\left(r_{1} s\right) J_{0} r_{2} s\right)}{s}=2 \int_{0}^{\infty} J_{0}\left(r_{1} s\right) J_{1}\left(r_{2} s\right) d s
$$

This integral is known as "Weber's discontinuous factor," and it is known that

$$
\begin{aligned}
\int_{0}^{\infty} J_{0}\left(r_{1} s\right) J_{1}\left(r_{2} s\right) d s & =\frac{2}{r_{2}} \ldots \text { if } r_{2}>r_{1} \\
& =0 \ldots \text { if } r_{2}<r_{1}
\end{aligned}
$$

This may be expressed as $-\frac{\partial}{\partial r_{2}} a_{y}\left(r_{2}, y_{2}\right)$, where $a_{y}$ is a finite and continuous vector potential given by

$$
\begin{aligned}
a_{y}\left(r_{2}, y_{2}\right) & =-2 \log r_{2} \text { if } r_{2}>r_{1} \\
& =-2 \log r_{1} \text { if } r_{2}<r_{1}
\end{aligned}
$$

(The passage from the Newtonian to the logarithmic vector potential amounts to discarding an infinite constant.) Hence, the axial component $A_{y}\left(r_{2} y_{2}\right)$ of the vector potential at any point $P_{2}\left(r_{2} y_{2}\right)$, due to the unit current in the infinite helical filament, is

$$
\begin{aligned}
A_{\mathrm{y}}\left(r_{2} y_{2}\right) & =4 \sum_{n=1}^{\infty} \cos \frac{n}{p}\left(y_{2}-y_{1}\right) \int_{0}^{\infty} \frac{s J_{\mathrm{n}}\left(r_{1} s\right) J_{\mathrm{n}}\left(r_{2} s\right)}{s^{2}+\frac{n^{2}}{p^{2}}} d s \\
& -2 \log r_{2} \text { or }-2 \log r_{1}
\end{aligned}
$$


according as $r_{2}$ is greater than or less than $r_{1}$. Now it has been shown in Appendix 6, equations (8) and (11), that

$$
\begin{gathered}
\int_{0}^{\infty} \frac{s J_{\mathbf{k}}\left(r_{1} s\right) J_{\mathbf{k}}\left(r_{2} s\right)}{s^{2}+\frac{n^{2}}{p^{2}}} d s=\frac{\pi i}{2} J_{\mathbf{k}}\left(\frac{i n r_{1}}{p}\right) H_{\mathbf{k}}{ }^{1}\left(\frac{i n r_{2}}{p}\right) \\
\approx \frac{p}{2 n \sqrt{r_{1} r_{2}}} e^{-n\left(\frac{r_{2}-r_{1}}{\mathbf{p}}\right)}
\end{gathered}
$$

\section{(independent of $k$ )}

provided $r_{2}>r_{1}$. The last form is correct to the second order inclusive, if $\frac{p}{r_{2}}$ and $\frac{r_{2}-r_{1}}{r_{2}}$ are small quantities of the first order. If $r_{2}<r_{1}$ then we must interchange $r_{1}$ and $r_{2}$ in the second member of (12). Consequently, if neither of the cylindrical radii $r_{1}$ and $r_{2}$ are infinitesimal, the axial component of vector potential, due to unit current in the infinite helical filament, is given by

$$
\begin{aligned}
A_{y}\left(r_{2} y_{2}\right) & =-2 \log r_{2}+\frac{2 p}{\sqrt{r_{1} r_{2}}} \sum_{n=1}^{\infty} \frac{e^{-n\left(\frac{r_{1}-r_{1}}{p}\right)}}{n} \cos \frac{n}{p}\left(y_{2}-y_{1}\right) \text { if } r_{2}>r_{1} \\
& =-2 \log r_{1}+\frac{2 p}{\sqrt{r_{1} r_{2}}} \sum_{n=1}^{\infty} \frac{e^{-n\left(\frac{r_{1}-r_{1}}{p}\right)}}{n} \cos \frac{n}{p}\left(y_{2}-y_{1}\right) \text { if } r_{2}<r_{1}
\end{aligned}
$$

or, if we let

$$
F_{2}(z)=\sum_{n=1}^{\infty} \frac{e^{-\mathrm{n} z}}{n}=-\log \left(1-e^{-z}\right)
$$

where

$$
z=r_{2}-r_{1}+i\left(y_{2}-y_{1}\right)
$$

Then (13) may be written

$$
\begin{aligned}
A_{\mathbf{y}}\left(r_{2}, y_{2}\right) & =\frac{2 p}{\sqrt{r_{1} r_{2}}} F_{2}\left(\frac{z}{p}\right)-2 \log r_{2} \text { if } r_{2}>r_{1} \\
& =\frac{2 p}{\sqrt{r_{1} r_{2}}} F_{2}\left(\frac{z}{p}\right)-2 \log r_{1} \text { if } r_{2}<r_{1}
\end{aligned}
$$

where the real part is to be taken. It is interesting to notice from (13) or (16) that since the pitch is small compared to $r_{1}$ or $r_{2}$, the series in (13) which is a periodic function of $y_{2}$ of period $2 \pi p$ becomes insignificant when the point $P_{2}$ is not very close to the filament, 
leaving the term $-2 \log r_{2}$ as the value of $A_{y}$ when $r_{2}>r_{1}$. This gives a magnetic field $H_{\theta}=\frac{2}{r_{2}}$ which is that due to a unit current flowing along the $y$ axis. If the point $P_{2}$ is within the helix, the magnetic field $H_{\theta}$ is practically zero. However, if $P_{2}$ comes very close to the helix, the series becomes logarithmically infinite, since as shown in Appendix 7, equation (8),

$$
\left.\begin{array}{c}
F_{2}\left(\frac{z}{p}\right)=-\log \frac{z}{p}+\frac{1}{2}\left(\frac{z}{p}\right)+\sum_{n=1}^{\infty} \frac{(-1)^{\mathrm{n}}}{n}\left(\frac{z}{2 \pi p}\right)^{2 \mathrm{n}} S_{2 \mathrm{n}} \\
\text { where } S_{\mathrm{n}}=\sum_{k=1}^{\infty} \frac{1}{k^{\mathrm{n}}}
\end{array}\right\}
$$

If we write

$$
z \equiv r_{2}-r_{1}+i\left(y_{2}-y_{1}\right)=R_{12} e^{i \phi_{1}}
$$

then

$F_{2}\left(\frac{z}{p}\right)_{\text {real part }}=-\log \frac{R_{12}}{p}+\frac{\left|r_{2}-r_{1}\right|}{2 p}+\sum_{n=1}^{\infty} \frac{(-1)^{\mathrm{n}}}{n}\left(\frac{R_{12}}{2 \pi p}\right)^{2 \mathrm{n}} \cos 2 n \phi_{12} S_{2 \mathrm{n}}$

However, if one desires to follow the corkscrew shape of the magnetic lines in the immediate neighborhood of the helical current filament, it is necessary to evaluate the other components of the vector potential. A similar treatment of the second equation of (5), taking account of the fact that the second form of (12) is independent of $k$, shows that to the same approximation

$$
A_{\mathrm{r}}\left(r_{2} y_{2}\right)=0
$$

Similarly, the first equation of (5) reduces to

$$
\begin{aligned}
A_{\theta}\left(r_{2} y_{2}\right) & =4 \sqrt{\frac{r_{1}}{r_{2}}} F_{2}\left(\frac{z}{p}\right)+\frac{r_{1}^{2}}{p r_{2}} \ldots \text { if } r_{2}>r_{1} \\
& =4 \sqrt{\frac{r_{1}}{r_{2}}} F_{2}\left(\frac{z}{p}\right)+\frac{r_{2}}{p} \text { if } r_{2}<r_{1}
\end{aligned}
$$

To examine the magnetic field of the helix, we may notice that at points which are not very close to the helix it is not periodic, but is equal to that produced by a current sheet in the cylinder $r=r_{1}$, in which there is unit current flowing in the direction of the axis and uniformly distributed, together with a uniform distribution of circular current, the total current circulating around unit length of the 
cylinder being $\frac{1}{2 \pi p}$, since $2 \pi p$ is the pitch or width of one turn. This part of the field is derived from a vector potential as follows:

$$
\left.\begin{array}{l}
H_{\mathrm{r}}^{\mathrm{g}}\left(r_{2}, y_{2}\right)=-\frac{\partial}{\partial y_{2}} A_{\theta}^{\mathrm{s}}\left(r_{2}, y_{2}\right) \\
H_{\theta}^{\mathrm{g}}\left(r_{2}, y_{2}\right)=-\frac{\partial}{\partial r_{2}} A_{\mathrm{y}}^{\mathrm{g}}\left(r_{2}, y_{2}\right) \\
H_{\mathrm{y}}^{\mathrm{B}}\left(r_{2}, y_{2}\right)=\frac{1}{r_{2}} \frac{\partial}{\partial r_{2}}\left(r_{2} A_{\theta}^{\mathrm{g}}\left(r_{2}, y_{2}\right)\right)
\end{array}\right\}
$$

where

$$
\left.\begin{array}{l}
A_{\mathrm{y}}^{\mathrm{g}}\left(r_{2} y_{2}\right)=-2 \log r_{2}, A_{\theta}^{\mathrm{g}}\left(r_{2}, y_{2}\right)=\frac{r_{1}{ }^{2}}{p r_{2}} \text { if } r_{2}>r_{1} \\
A_{\mathrm{y}}^{\mathrm{g}}\left(r_{2} y_{2}\right)=-2 \log r_{1}, A_{\theta}^{\mathrm{s}}\left(r_{2}, y_{2}\right)=\frac{r_{2}}{p} \text { if } r_{2}<r_{1}
\end{array}\right\}
$$

That is, making use of (22) in (21)

$$
\left.\begin{array}{l}
H_{\mathrm{r}}^{\mathrm{s}}=0, H_{\theta}^{\mathrm{s}}=\frac{2}{r_{2}}, H_{\mathrm{y}}^{\mathrm{g}}=0 \text { if } r_{2}>r_{1} \\
H_{\mathrm{r}}^{\mathrm{g}}=0, H_{\theta}^{\mathrm{s}}=0, H_{\mathrm{y}}^{\mathrm{s}}=4 \pi \quad\left(\frac{1}{2 \pi p}\right) \text { if } r_{2}<r_{1}
\end{array}\right\}
$$

In the case of the helix there is superposed upon this current-sheet field $H^{\mathrm{s}}$ a periodic field $H^{\mathrm{p}}$, which is negligible compared to the former at appreciable distances from the helical filament, but which becomes the predominating part of the field in the immediate neighborhood of the helix. Its value at a point $P_{2}\left(r_{2} y_{2}\right)$ is given by

$$
\left.\begin{array}{l}
H_{\mathbf{r}}^{\mathrm{p}}=-4 \sqrt{\frac{r_{1}}{r_{2}}} \frac{\partial}{\partial y_{2}} F_{2}\left(\frac{z}{p}\right) \\
H_{\theta}^{\mathrm{p}}=-\frac{2 p}{\sqrt{r_{1}}} \frac{\partial}{\partial r_{2}}\left(\frac{1}{\sqrt{r_{3}}} F_{2}\left(\frac{z}{p}\right)\right) \\
H_{\mathbf{y}}{ }^{\mathrm{p}}=\frac{4 \sqrt{r_{1}}}{r_{2}} \frac{\partial}{\partial r_{2}}\left(\sqrt{r_{2}} F_{2}\left(\frac{z}{p}\right)\right)
\end{array}\right\}
$$

where the real part is to be taken, and

$$
z=\left|r_{2}-r_{1}\right|+i\left(y_{2}-y_{1}\right)
$$

The only places where this field is appreciable are those in which $z$ is small compared to $r_{1}$ or $r_{2}$; that is, where $z$ is of the same order as the small quantity $p$ or smaller, so that $\frac{z}{p}$ is either finite or very small (but not large). In this case 


$$
\frac{\partial}{\partial r_{2}}\left(\frac{F_{2}\left(\frac{z}{p}\right)}{\sqrt{r_{2}}}\right)=\frac{F^{\prime}\left(\frac{z}{p}\right)}{p \sqrt{r_{2}}} \text { minus a term } \frac{F_{2}\left(\frac{\psi}{p}\right)}{2 r_{2}^{3 / 2}}
$$

which is negligible in comparison with the first.

The field $H^{\mathrm{p}}$ given by (24) is independent of $\theta_{2}$ so that a study of it in the $x y$ plane $\theta_{2}=o$ gives a typical picture for any other plane $\theta_{2}=$ constant. If we let $r_{2}=x_{2}$, then when $P_{2}$ is in the neighborhood of the filament the periodic part of the magnetic field is given by

$$
\left.\begin{array}{l}
H_{\mathrm{x}}^{\mathrm{p}}\left(x_{2} y_{2}\right)=\frac{\partial \psi^{\mathrm{p}}}{\partial y_{2}}, H_{\mathrm{y}}^{\mathrm{p}}\left(x_{2} y_{2}\right)=-\frac{\partial \psi^{\mathrm{p}}}{\partial x_{2}} \\
H_{\theta}^{\mathrm{D}}\left(x_{2} y_{2}\right)=\frac{2 p}{r_{1}} H_{\mathrm{y}}^{\mathrm{p}}\left(x_{2} y_{2}\right)
\end{array}\right\}
$$

where

$$
\psi^{\mathrm{p}}=4 \log \left[1-e-\frac{\left|\mathrm{x}_{1}-\mathrm{x}_{1}\right|+\mathrm{i}\left|\mathrm{y}_{1}-\mathrm{y}_{1}\right|}{\mathrm{p}}\right]_{\text {real part }}
$$

If we take a new system of $x$ and $y$ axes parallel to the old ones but with their origin in the helix, then a point in the neighborhood of the origin has the coordinates

and if $x>0$

$$
x=x_{2}-x_{1} \quad y=y_{2}-y_{1}
$$

$$
\psi^{\mathfrak{p}}=2 \log \left[1-2 e^{-\frac{\mathrm{x}}{\mathrm{p}}} \cos \frac{y}{p}+e^{-\frac{2 \mathrm{x}}{\mathrm{p}}}\right]
$$

Since $H_{\mathrm{x}}^{\mathrm{p}}=\frac{\partial \psi^{\mathrm{p}}}{\partial y}$ and $H_{\mathrm{y}}^{\mathrm{p}}=-\frac{\partial \psi^{\mathrm{p}}}{\partial x}$ it is evident that $\psi^{\mathrm{p}}$ is the magnetic flux function for that part of the periodic magnetic field near the helix which lies in the $x y$ plane. The $x$ and $y$ components of the nonperiodic or "current sheet" part of the magnetic field may also be expressed in terms of a flux function $\psi^{8}$. In fact, the equations (23) become with the new system of axes

where

$$
H_{\mathrm{x}}=\frac{\partial \psi^{\mathrm{s}}}{\partial y}, H_{\mathrm{y}}=-\frac{\partial \psi^{\mathrm{s}}}{\partial x}
$$

$$
\begin{aligned}
\psi^{8} & =0 \text { if } x>0 \\
& =-\frac{2 x}{p} \text { if } x<0
\end{aligned}
$$

Hence the resultant field is derivable from the flux function

$$
\psi=\psi^{\mathrm{B}}+\psi^{\mathrm{p}}
$$

The magnetic lines of force near the helix may be plotted from their equations

$$
\psi=2 \log \left[1-2 e^{-\frac{x}{p}} \cos \frac{y}{p}+e^{-\frac{2 x}{p}}\right]+\psi^{s}=\text { constant }
$$


These lines of magnetic force are identical with the equipotential lines due to a grating of parallel wires in one plane in the presence of an electric field. They are shown in Figure 13 of Volume I of Maxwell's Electricity and Magnetism in connection with the electrostatic problem which is mathematically identical with the one here considered.

\section{Appendix 11.-CORRECTIONS DUE TO LEAD-IN WIRES}

The inductance of the helix and lead-in wires together is

$$
L=L_{\mathrm{h}}+L_{1}+2 M_{\mathrm{lh}}
$$

To compute the mutual inductance $M_{\mathrm{n}}$ between the helix and lead wires, we may assume that the latter are linear and lie in plane $y=0$. (See fig. 5.) The helix may be idealized as a continuous cylindrical

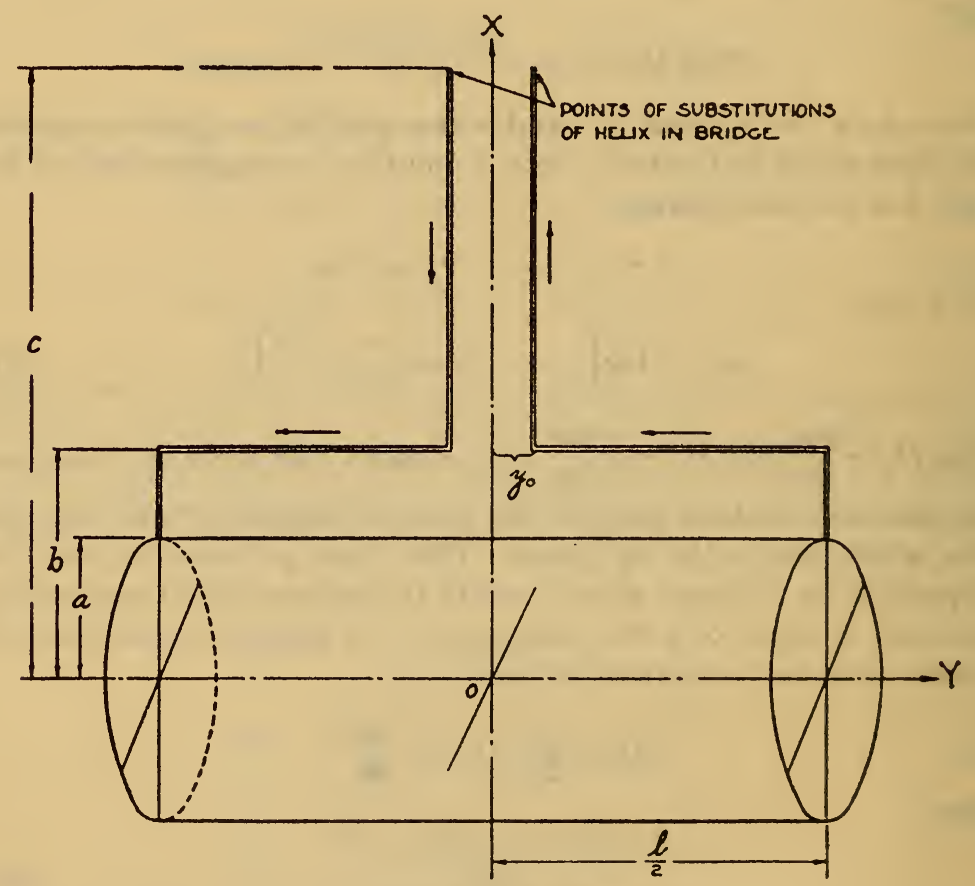

Fig. 5.-Arrangement of lead wires

current sheet around which the current winds in helical lines; that is, we may consider the coil as consisting of tape windings without insulation between strips. Since each strip carries unit current, the total flow in the $y$ direction is everywhere constant and equal to one, and the surface density of current has a $y$ component $\frac{1}{2 \pi a}$ and a circular component of amount $\frac{1}{\beta}=\frac{N}{7}=\frac{1}{2 \pi p}$. 
It is evident that those leads which are perpendicular to the axis of the cylindrical sheet have no mutual inductance with the latter, so that

$$
\begin{aligned}
2 M_{\mathrm{n} 1} & =-2 \int_{\mathrm{y}_{0}}^{\frac{l}{2}} d y_{1}\left[A_{\mathrm{y}}{ }^{\mathrm{h}}\left(b, y_{1}, o\right)-A_{\mathrm{y}}{ }^{\mathrm{h}}\left(b,-y_{1}, o\right]\right. \\
& =-4 \int_{\mathrm{y}_{0}}^{\frac{l}{2}} A_{\mathrm{y}}{ }^{\mathrm{h}}\left(b, y_{1}, o\right) d y_{1}
\end{aligned}
$$

where the $y$ component of the vector potential at any point $\left(b, y_{1}, o\right)$ due to the current sheet, is given by

$$
\begin{aligned}
& A_{\mathrm{y}}{ }^{\mathrm{h}}\left(b, y_{1}, o\right)=\frac{1}{2 \pi} \int_{-\frac{l}{2}}^{\frac{l}{2}} d y_{2} \int_{0}^{2 \pi} \frac{d \phi}{\sqrt{a^{2}+b^{2}-2 a b \cos \phi+\left(y_{2}-y_{1}\right)^{2}}} \\
= & \frac{1}{\pi} \int_{-\frac{l}{2}}^{\frac{l}{2}} d y_{2} \int_{0}^{2 \pi} d \phi \sum_{n=0}^{\infty} \epsilon_{\mathrm{n}} \int_{0}^{\infty} d s e^{-\left|y_{2}-\mathrm{y}_{\mathrm{y}}\right| \mathrm{s}} J_{\mathrm{n}}(a s) J_{\mathrm{n}}(b s) \cos n \phi b y(4) \\
= & \int_{-\frac{l}{2}}^{\frac{l}{2}} d y_{2} \int_{0}^{\infty} d s e^{-\left|y_{s}-\mathrm{y}_{1}\right| s} J_{0}(a s) J_{0}(b s) \\
= & \int_{0}^{\infty} d s J_{0}(a s) J_{0}(b s)\left[\frac{2-e^{-\left(\frac{l}{2}+\mathrm{y}_{1}\right) \mathrm{s}}-e^{-\left(\frac{l}{2}-\mathrm{y}_{1}\right)}}{s}\right]
\end{aligned}
$$

Substituting this value of $A_{\mathrm{y}}{ }^{\mathrm{b}}\left(b, y_{1}, o\right)$ in (2) and integrating gives

$$
2 M_{\mathrm{h} 1}=-4\left\{F(l)+F\left(\frac{l}{2}-y_{0}\right)-F\left(\frac{l}{2}+y_{0}\right)\right\}
$$

where

$$
\begin{aligned}
F(l) & =\int_{0}^{\infty}\left[\frac{e^{-1 s}+l s-1}{s^{2}}\right] J_{0}(a s) J_{0}(b s) d s \\
& =\int_{0}^{l} d \lambda^{1} \int_{0}^{\lambda^{1}} d \lambda \int_{0}^{\infty} d s e^{-\lambda s} J_{0}(a s) J_{0}(b s) \\
& =\frac{1}{\pi \sqrt{a b}} \int_{0}^{l} d \lambda^{1} \int_{0}^{\lambda d} d \lambda \mu_{\lambda} K\left(\mu_{\lambda}\right)=\frac{1}{\pi \sqrt{a b}} \int_{0}^{l} d \lambda(l-\lambda) \mu_{\lambda} K\left(\mu_{\lambda}\right)
\end{aligned}
$$

where

$$
\frac{1}{\mu_{\lambda}^{2}}=\frac{1}{\mu_{0}^{2}}+\left(\frac{\lambda}{2 \sqrt{a b}}\right)^{2} \text { and } \frac{1}{\mu_{0}^{2}}=1+\left(\frac{b-a}{2 \sqrt{a b}}\right)^{2}
$$


Changing the variable of integration from $\lambda$ to $\mu$ gives

$$
F(l)=\frac{2 l \mu_{0}}{\pi}\left\{\int_{\mu_{1}}^{\mu 0} \frac{K(\mu) d \mu}{\mu \sqrt{\mu_{0}^{2}-\mu^{2}}}-\frac{\mu_{1}}{\sqrt{\mu_{0}^{2}-\mu_{1}^{2}}} \int_{\mu_{1}}^{\mu_{0}} \frac{K(\mu)}{\mu^{2}} d \mu\right\}
$$

where

$$
\frac{1}{\mu_{1}^{2}}=\frac{1}{\mu_{0}^{2}}+\left(\frac{l}{2 \sqrt{a b}}\right)^{2}
$$

But

$$
\int \frac{K(\mu)}{\mu^{2}} d \mu=-\frac{E(\mu)}{\mu}+\text { constant }
$$

Hence

$$
F(l)=\frac{2 l \mu_{0}}{\pi}\left\{\int_{\mu_{1}}^{\mu_{0}} \frac{K(\mu) d \mu}{\mu \sqrt{\mu_{0}^{2}-\mu^{2}}}+\frac{\mu_{0}}{\sqrt{\mu_{0}^{2}-\mu_{1}^{2}}}\left(\frac{E\left(\mu_{0}\right)}{\mu_{0}}-\frac{E\left(\mu_{1}\right)}{\mu_{1}}\right)\right\}
$$

If $l, y_{0}, a$, and $b$ are numerically given, this equation enables one to evaluate $F(l)$ by mechanical integration. However, the integrand of the integral in (9) becomes infinite at the upper limit $\mu=\mu_{0}$. Hence it must be put in a form more convenient for a graphical integration. This may be done as follows:

$$
\int_{\mu_{1}}^{\mu_{0}} \frac{K(\mu) \mu d \mu}{\mu^{2} \sqrt{\mu_{0}^{2}-\mu^{2}}}=\frac{\sqrt{\mu_{0}^{2}-\mu_{1}^{2}}}{\mu_{1}^{2}} K\left(\mu_{1}\right)+\int_{\mu_{1}}^{\mu_{0}} \sqrt{\mu_{0}^{2}-\mu^{2}} \frac{d}{d \mu}\left(\frac{K(\mu)}{\mu^{2}}\right) d \mu
$$

by integration of parts

$$
=\frac{\sqrt{\mu_{0}^{2}-\mu_{1}^{2}}}{\mu_{1}^{2}} K\left(\mu_{1}\right)+\int_{\mu_{1}}^{\mu_{0}} \frac{\sqrt{\mu_{0}^{2}-\mu^{2}}}{\mu^{3}}\left\{\frac{E(\mu)}{1-\mu^{2}}-3 K(\mu)\right\} d \mu
$$

Hence (9) may be written

$$
\begin{aligned}
F(l) & =\frac{2 l \mu_{0}}{\pi}\left\{\int_{\mu_{1}}^{\mu_{0}} \frac{\sqrt{\mu_{0}^{2}-\mu^{2}}}{\mu^{3}}\left[\frac{E(\mu)}{1-\mu^{2}}-3 K(\mu)\right] d \mu\right. \\
& \left.+\frac{\sqrt{\mu_{0}^{2}-\mu_{1}^{2}}}{\mu_{1}^{2}} K\left(\mu_{1}\right)+\frac{\mu_{0}}{\sqrt{\mu_{0}^{2}-\mu_{1}^{2}}}\left(\frac{E\left(\mu_{0}\right)}{\mu_{0}}-\frac{E\left(\mu_{1}\right)}{\mu_{1}}\right)\right\}
\end{aligned}
$$

If $a$ and $b$ are fixed, $\mu_{0}$ is determined. Hence the integral in this expression (11) may be drawn by means of an integraph and plotted as a function of $\mu_{1}$ and also plotted as a function of $l$. From the latter curve, together with a table of elliptic integrals, the values of $F(l), F\left(\frac{l}{2}-y_{0}\right)$, and $F\left(\frac{l}{2}+y_{0}\right)$ may be readily obtained, so that the term $2 M_{\mathrm{hl}}$ given by (4) may be computed. 
It is interesting to note that if $b-a$ is very small, as well as $y_{0}$, the return leads being very close to the solenoid, $\mu_{0}$ approaches 1 and $\mu_{1}$ approaches $k$, so that approximately

$$
\begin{aligned}
2 M_{\mathrm{bl}} & =-4 F(l)=-\frac{4}{\pi a} \int_{0}^{1} d \lambda_{1} \int_{0}^{\lambda_{1}^{1}} d \lambda \mu_{\lambda} K\left(\mu_{\lambda}\right) \\
& =-\frac{8 a}{\pi} \int_{0}^{\frac{\sqrt{1-k_{2}}}{\mathrm{k}}} d y_{2} \int_{0}^{y_{2}} d y_{1} \frac{K\left(\frac{1}{\sqrt{1+y_{1}^{2}}}\right)}{\sqrt{1+y_{1}{ }^{2}}}=-\frac{8 a}{\pi} B_{0}
\end{aligned}
$$

It was shown in Appendix 9 that $+\frac{8 a B_{0}}{\pi}$ was the part of $A_{2}(k)$ which represented the contribution to the self-inductance due to the axial component of current in the helix. It is evident, therefore, that the return leads when very close to the solenoid just cancel this term by their mutual inductance.

An important practical conclusion may be drawn from this. It is found from Figure 2 that for a coil $40 \mathrm{~cm}$ long, of mean radius $15 \mathrm{~cm}$, so that $k=0.36, B_{0}$ has the value 2.5 , so that the term $\frac{8 a B_{0}}{\pi}=100 \mathrm{~cm}$ approximately, which is $1 / 200000$ of the inductance of the whole. The important conclusion may therefore be drawn that if the return leads are close to the solenoid, as in Figure 5, the correction due to their. mutual inductance with the helix may be taken as just $-\frac{8 a B_{0}(k)}{\pi}$. This term is plotted in Figure 2.

The remaining correction term is $L_{e}$, the inductance of the leads. This may be computed by familiar methods in terms of their length, diameter, and their mutual inductance with each other, the latter computed as if they were linear.

Washington, February 25, 1926. 